Aus der Klinik und Poliklinik für Psychiatrie und Psychotherapie Klinik der Ludwig-Maximilians-Universität München Vorstand: Prof. Dr. Peter G. Falkai

\title{
Stressreagibilität bei Alkoholabhängigen unter Berücksichtigung von Abstinenzdauer und Krankheitsschwere
}

Dissertation zum Erwerb des Doktorgrades der Humanbiologie an der Medizinischen Fakultät der Ludwig-Maximilians-Universität zu München vorgelegt von Ilija Tolic

aus

Mala Socanica

Jahr 2020 
Mit Genehmigung der Medizinischen Fakultät der Universität München

Berichterstatter: Prof. Dr. med. M. Soyka

Mitberichterstatter: Prof. Dr. rer. nat. Frank Mußhoff

Prof. Dr. med. Wolfgang Eisenmenger

Prof. Dr. med. Markus Backmund

Dekan Prof. Dr. med. dent. Reinhard Hickel

Tag der mündlichen Prüfung: $\quad$ 22.09.2020 


\section{Danksagung}

Herrn Professor Dr. med. Michael Soyka möchte ich an dieser Stelle herzlich für die sehr geduldige und hervorragende Betreuung danken. Ein ebenso großes und herzliches Dankeschön gilt meinem Supervisor Dr. Heinrich Küfner, der mich jahrelang während meiner therapeutischen Arbeit unterstützt hat. Beim Team des BlaukreuzZentrums München bedanke ich mich für die tatkräftige Unterstützung bei der komplexen Durchführung der Studie und das hervorragende Arbeitsklima. Bei dem Personal der Station C4 bedanke ich mich für die kurzzeitige Rekrutierung der Patienten. Zuletzt möchte ich mich noch bei meiner Ehefrau Katharina und meinen Kindern Patricia und Marin-Eugen bedanken, die mich bedingungslos und liebevoll während der gesamten Dissertation unterstützt haben. Ihnen widme ich diese Arbeit. 


\section{Inhaltsverzeichnis}

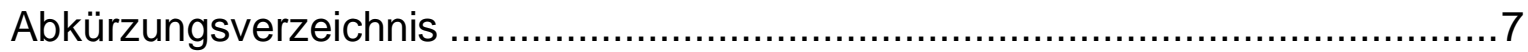

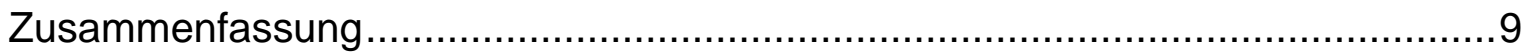

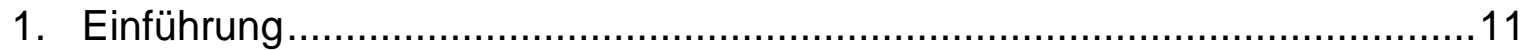

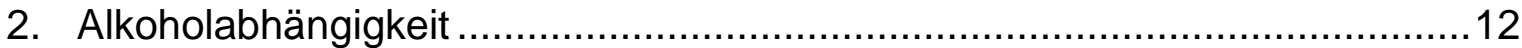

2.1 Begriffsklärung und Diagnostik im Kontext einer zeitlichen Perspektive und der kategorialen Vielfalt: Alkoholismus, Missbrauch, Abhängigkeit und

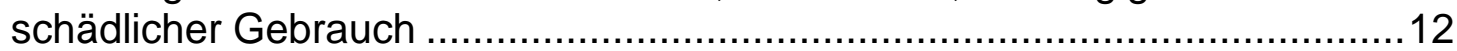

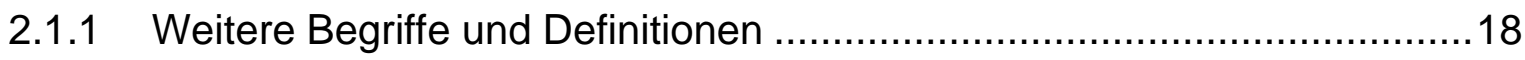

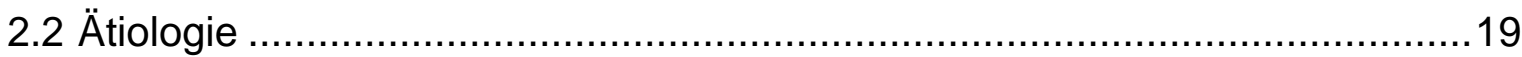

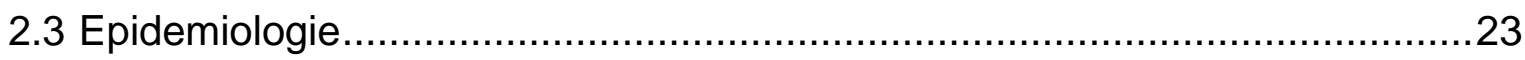

2.4 Somatische und psychische Folgeschäden des Alkoholismus......................24

2.5 Alkoholismus und Auswirkungen auf das endokrine System ........................26

2.5.1 Alkohol und Hypothalamus-Hypophysen-Nebennierenrinden-Achse

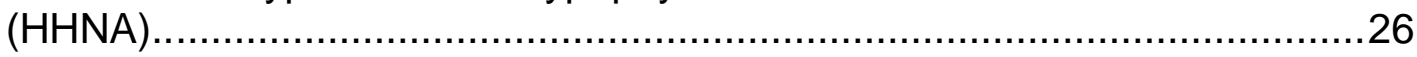

2.6 Alkoholabhängigkeit und multipler Substanzgebrauch (Mehrfachabhängigkeit)

2.7 Alkoholabhängigkeit und Komorbidität mit psychischen Störungen ohne

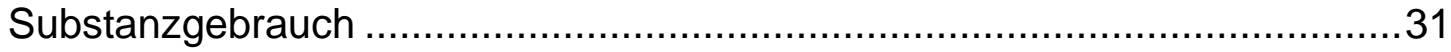

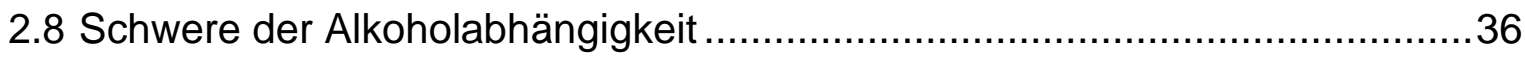

2.8.1 European Addiction Severity Index (EuropASI) ……...........................37

2.8.2 Skala zur Erfassung der Schwere der Alkoholabhängigkeit (SESA) ........37

2.8.3 Severity of Alcohol Dependence Questionnaire (SADQ) ........................38

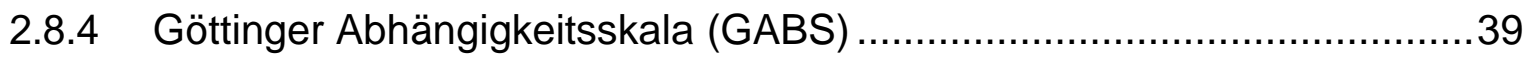

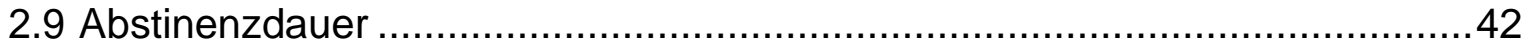

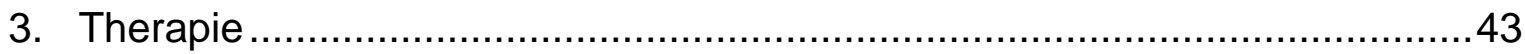

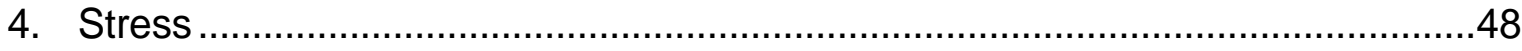

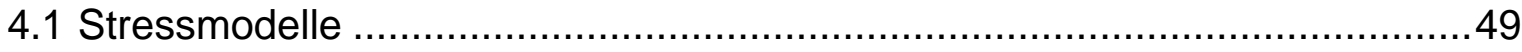

4.2 Stressreaktion: Darstellung des Ablaufs und der beteiligten biologischen

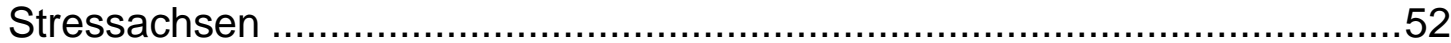

4.2.1 Das Sympatho-adrenomedulläre System (SAM) ….....................53

4.2.2 Hypothalamus-Hypophysen-Nebennierenrinden-Achse (HHNA) .............55

4.2.2.1 Determinanten der HHNA-Aktivität ..................................................58

4.2.2.2 Wechselwirkungen zwischen den Stresssystemen der HHNA und SAM 
4.3 Stress, frühe Stresserfahrungen und Folgen für die Gesundheit: Stress als Initiator und Moderator

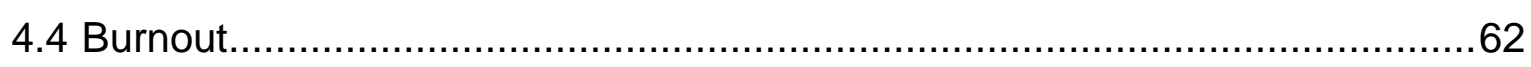

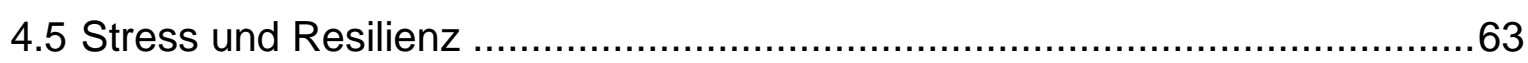

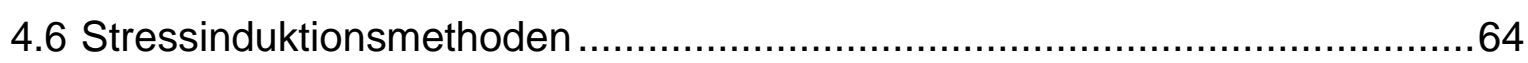

4.7 Erfassungen biologischer Variablen der Stressreaktion........................67

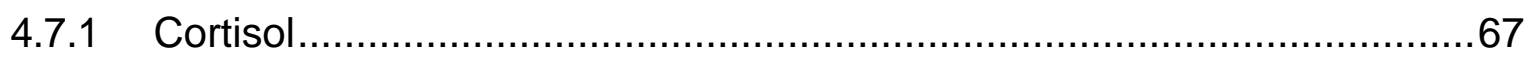

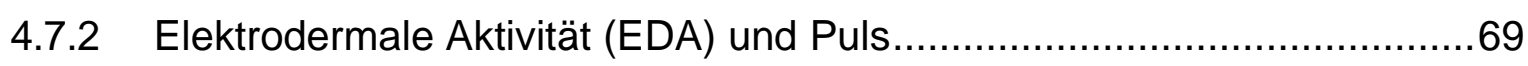

5. Erhebungsinstrumente: Diagnostik und Psychopathologie .........................70

5.1 AUDIT-Fragebogen (Alcohol Use Disorders Identification Test) …................71

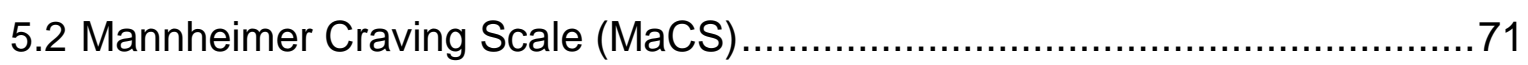

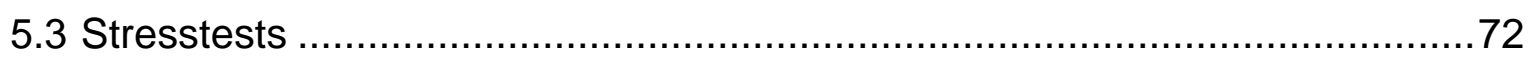

5.3.1 Der Stressverarbeitungsfragebogen 120 (SVF 120) …........................72

5.3.2 Trierer Inventar zum chronischen Stress (TICS) ................................72

5.3.3 Inventar zur Erfassung Lebensverändernder Ereignisse (ILE) .................73

5.3.4 Alltagsbelastungsfragebogen (ABF) .............................................. 73

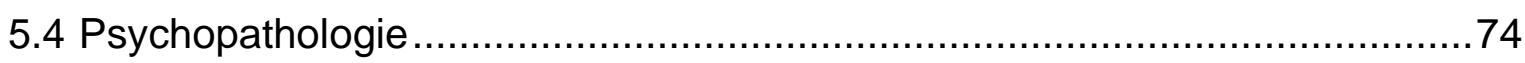

5.4.1 State-Trait-Angstinventar (STAI) und Becks-Depressions-Inventar II

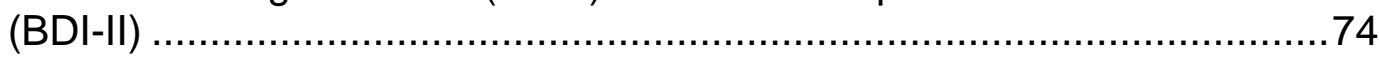

5.4.2 Strukturiertes Klinisches Interview für DSM-IV (SKID-II) ........................74

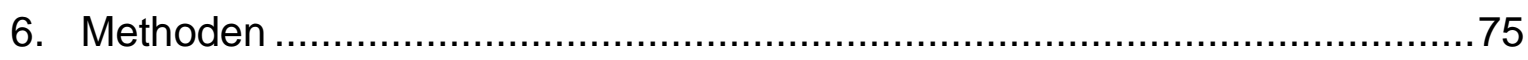

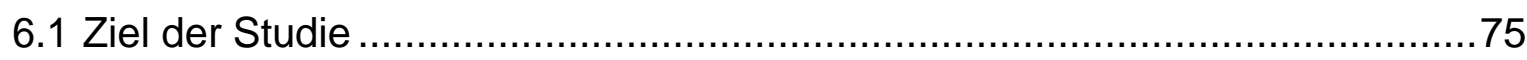

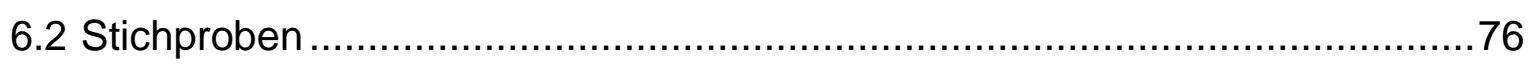

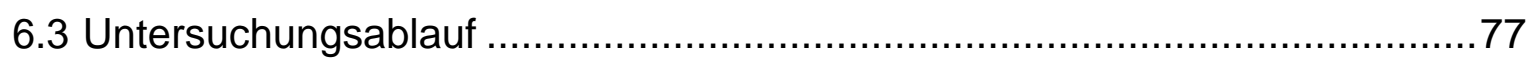

6.4 Erfassungen der Stressreaktionen ....................................................

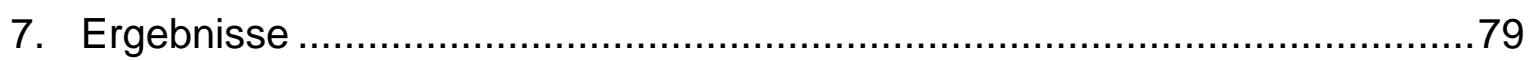

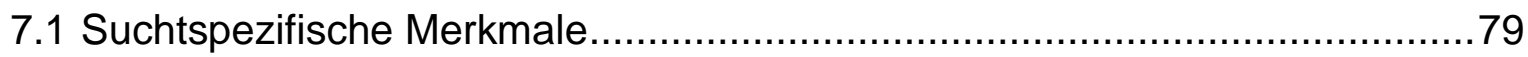

7.2 Psychopathologie (Angst, Depression, einschneidende Lebensereignisse,

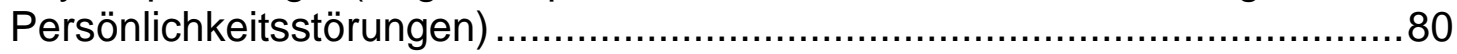

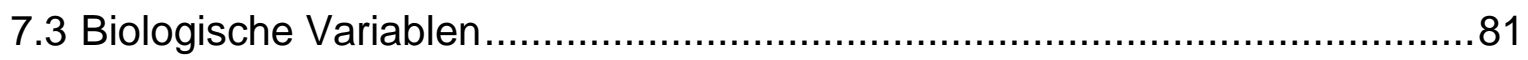

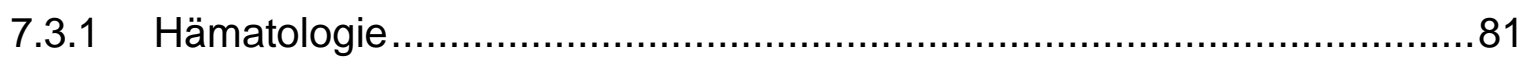

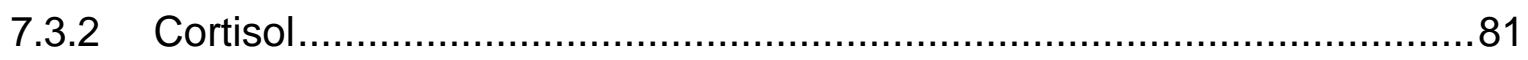

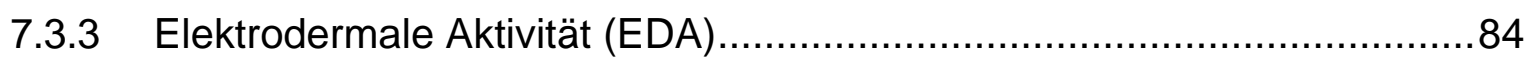

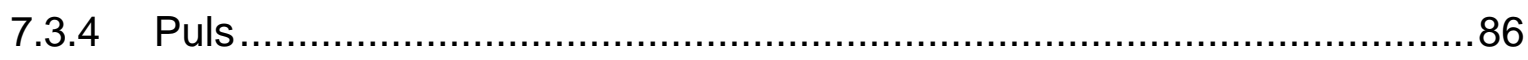

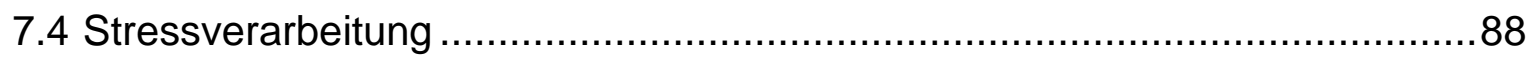

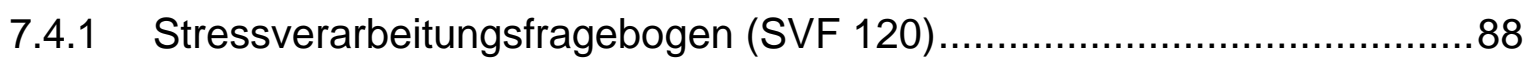

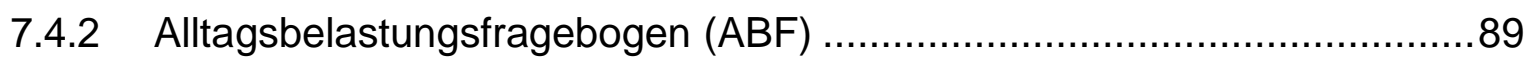


7.4.3 Trierer Inventar zum chronischen Stress (TICS) ............................. 89

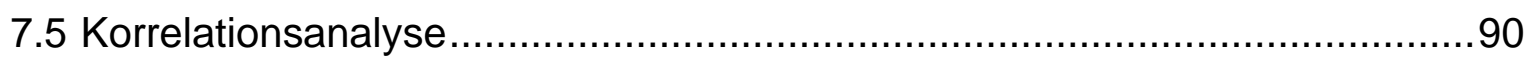

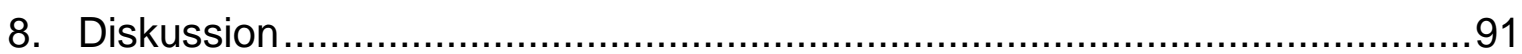

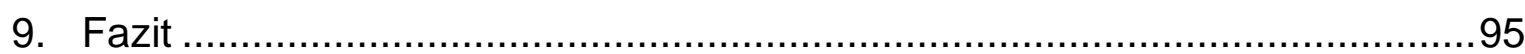

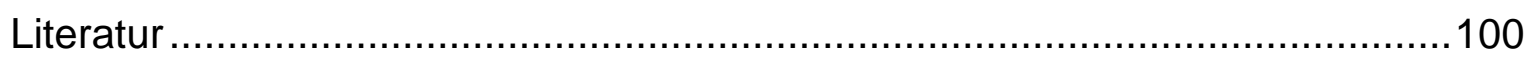

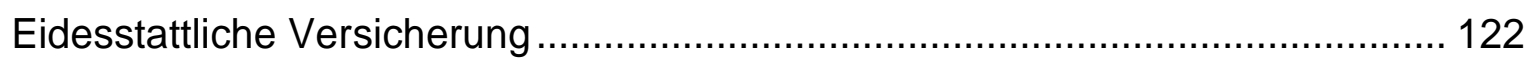

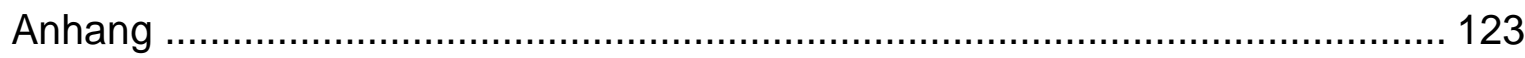




\section{Abkürzungsverzeichnis}

ABF

ACTH

ADH-4

ADHS

AUDIT

AVP

AWMF

BDI II

CAMP

CDT

CHRM-2

CPT

$\mathrm{CRH}$

CRHR1

DHS

DSM-IV und 5

EDA

EuropASI

FSH

GABA

GABRA-2

GABS

Gamma- GT

GOT

GPT

GR

HHGA

HHNA

HHSA

HHWA

ICD-10

ILE
Alltagsbelastungsfragebogen

Adrenokortikotropes Hormon

Alkoholdehydrogenase- 4

Aufmerksamkeits-Defizit-Hyperaktivitäts-

Syndrom

Alcohol Use Disorders Identification Test

Arginin-Vasopressin

Arbeitsgemeinschaft der Wissenschaftlichen Medizinischen Fachgesellschaften

Becks-Depressions-Inventar II

Cyclisches Adenosinmonophosphat

Carbohydrat-defizientes Transferrin

Acetylcholinerger Rezeptor Muscarin-2

Cold Pressor Test

Corticotropin-Releasing-Hormon

Corticotropin-Releasing-Hormon-

Rezeptor 1

Deutsche Hauptstelle für Suchtfragen

Diagnostisches und Statistisches Manual

Elektrodermale Aktivität

European Addiction Severity Index

Folikelstimulierendes Hormon

Gamma-Aminobuttersäure

Gamma-Aminobuttersäure A Rezeptor Alpha- 2

Göttinger Abhängigkeitsskala

Gamma-Glutamyltransferase

Glutamat-Oxalacetat-Transaminase

Glutamat-Pyruvat-Transaminase

Glukokortikoidrezeptor

Hypothalamus-Hypophysen-Gonaden-Achse

Hypothalamus-Hypophysen-Nebennierenrinden-Achse

Hypothalamus-Hypophysen-Schilddrüsen-Achse

Hypothalamus-Hypophysen-Wachstumshormon-Achse

International Classification of Diseases

Inventar zur Erfassung lebensverändernder Ereignisse 


\begin{tabular}{|c|c|}
\hline LC & Locus coeruleus \\
\hline $\mathrm{LH}$ & Luteinisierendes Hormon \\
\hline MaCS & Mannheimer Craving Scale \\
\hline MC2R & Melanocortin-2 Rezeptor \\
\hline mPFC & medialer präfrontaler Cortex \\
\hline MCV & mean corpuscular volume \\
\hline MR & Mineralokortikoidrezeptor \\
\hline NAA & N-Acetyl-Aspartat \\
\hline NTS & Nucleus tractus solitarii \\
\hline POMC & Proopiomelanocortin \\
\hline PTBS & Posttraumatische Belastungsstörung \\
\hline PVN & Paraventrikulärer Kern des Hypothalamus \\
\hline SADQ & Severity of Alcohol Dependence Questionnaire \\
\hline SAM & Sympatho-adrenomedulläres System \\
\hline SESA & Skala zur Erfassung der Schwere der Alkoholabhängigkeit \\
\hline SKID II & Strukturiertes Klinisches Interview für DSM-IV \\
\hline STAI & State-Trait-Angstinventar \\
\hline SVF 120 & Stressverarbeitungsfragebogen 120 \\
\hline TICS & Trierer Inventar zum chronischen Stress \\
\hline $\mathrm{TRH}$ & Thyreotropin-Releasing-Hormon \\
\hline $\mathrm{TSH}$ & Thyreo-stimulierendes Hormon \\
\hline TSST & Trier Sozial Stress Test \\
\hline VmPFC & Ventromedialer präfrontaler Kortex \\
\hline VS & Ventrales Striatum \\
\hline VTA & Ventrales tegmentales Areal \\
\hline WHO & World Health Organization \\
\hline ZNS & Zentrales Nervensystem \\
\hline
\end{tabular}




\section{Zusammenfassung}

\section{Stressreagibilität bei Alkoholabhängigen unter Berücksichtigung von Abstinenzdauer und Krankheitsschwere}

Hintergrund: Bei der Entstehung und Aufrechterhaltung von Alkoholabhängigkeit spielt Stress als Risikofaktor eine wichtige Rolle. Die Berücksichtigung von Stressreaktions- und Stressverarbeitungsmechanismen bei Alkoholabhängigen ist für die Aufrechterhaltung von Abstinenz im Alltag von Relevanz. Ebenso wichtig ist die Berücksichtigung von Krankheitsschwere, die ihrerseits Einfluss auf die Abstinenz, insbesondere auf das Verlangen nach Alkohol, ausübt. In zahlreichen Studien wurde die Abstinenzdauer, nicht aber die Krankheitsschwere ausreichend berücksichtigt. Ziel: Untersucht wird, ob neben der Abstinenzdauer auch die Schwere der Alkoholabhängigkeit einen Einfluss auf die Stressreagibilität und Stressverarbeitung hat. Zudem soll geprüft werden, bei welchen Patienten sich der Einfluss zeigt. Die Ergebnisse könnten mehr Klarheit zur komplexen bidirektionalen Beziehung zwischen Alkoholabhängigkeit und Stress bringen, aber auch für die Behandlung von Betroffenen nützlich sein. Methoden: Im Querschnittsdesign wurden 64 Alkoholabhängige und 30 gesunde Probanden untersucht. Die Patientenstichprobe wurde nach Abstinenzdauer von 7 und 70 Tagen in jeweils zwei Gruppen aufgeteilt. Ebenso nach Krankheitsschwere in leicht-mittelschwer und schwer. Mit dem Trier Sozial Stress Test (TSST) wurden alle Probanden akutem Stress ausgesetzt. Erhoben wurden Parameter wie Cortisol im Speichel, elektrodermale Aktivität (EDA), Puls, suchtspezifische Variablen (Schwere der Abhängigkeit, Trinkdauer, Trinkmenge, Suchtdruck, Leberenzyme, familiäre Vorgeschichte, Anzahl der Therapien, Rauchen, u. v. m.), psychopathologische Variablen (Angst, Depression, kritische Lebensereignisse, Persönlichkeitsstörungen), akute und chronische Stressverarbeitung, Alltagsbelastungen. Ergebnisse: Schwerkranke Patienten reagieren schwächer und etwas verzögert auf Stress, zeigen dafür aber eine verlängerte Stressreaktion im Vergleich $\mathrm{zu}$ leicht-mittelschwerkranken und Gesunden. Dies ist unabhängig von der Abstinenzdauer (7 oder 70 Tage). Schwerkranke zeigten stärkere Fluchtund Vermeidungsreaktionen, starkes Bedürfnis nach sozialer Unterstützung und die intensive gedankliche Weiterbeschäftigung als akute Stressbewältigungsstile. Als Zeichen chronischer Stressbelastung wurden erhöhte Werte in den Bereichen Mangel an sozialer Anerkennung, soziale Spannungen und Isolation festgestellt. Außerdem zeigten Schwerkranke stärkere familiäre Vorbelastung, stärkeren Suchtdruck, höhere Ängstlichkeit, längere Trinkdauer und mehr einschneidende Lebensereignisse als Leicht-mittelschwerkranke. Schlussfolgerungen: Für eine differenzierte Beurteilung von Stressreagibilität und Stressverarbeitung bei Alkoholabhängigkeit scheint neben Abstinenzdauer auch die Krankheitsschwere eine wichtige Rolle $\mathrm{zu}$ spielen. Weitere Anstrengungen sind nötig, um die Ergebnisse in die Behandlungsprogramme zu implementieren.

Schlüsselwörter: Alkoholabhängigkeit, Stress, Abstinenzdauer, Krankheitsschwere, Cortisol, EDA, Puls, TSST. 


\section{Abstract \\ Stress Responsiveness in Persons with Alcohol Addiction in Consideration of Abstinence Duration and Disease Severity}

Background: Stress increases the risk of developing and maintaining alcohol addiction. Mechanisms for reacting to, and coping with, stress in persons with alcohol addiction are an important factor enabling them to maintain abstinence in everyday life. Also of relevance for abstinence, and in particular craving for alcohol, is the severity of the dependence. Numerous studies have focused on the duration of abstinence, but not on the severity of the disease. Objective: This study investigates if, in addition to abstinence duration, also the severity of the alcohol addiction influences stress responsiveness and ability to cope with stress. Another objective is to ascertain in which patients such an influence can be found. The results can shed light on the complex bidirectional relationship between alcoholism and stress, but are also potentially useful in treating patients with this disease. Methods: In a cross-sectional design, 64 alcohol-dependent patients and 30 healthy controls were examined. The alcoholics sample was divided into two groups according to a duration of abstinence of seven days and 70 days. Each group was further divided into a subgroup of individuals with mild to moderate alcohol addiction and a subgroup of patients with severe alcohol addiction. Using the Trier Social Stress Test (TSST), all participants were subjected to acute stress. Parameters measured were salivary cortisol, skin conductance response, heart rate, addiction-related variables (addiction severity, duration of alcohol abuse, drinking quantity, craving, liver enzymes, family history of alcoholism, number of therapies, smoking), psychopathological variables (anxiety, depression, live events, personality disorders), acute and long-term stress coping, and level of strain on everyday life. Results: In patients with severe addiction, the reaction to stress is weaker and slightly retarded, but also of a longer duration than that patients with mild and moderate addiction and of healthy controls. This is independent of the length of abstinence ( 7 days and 70 days). In severely ill patients, strategies for coping with acute stress differed from those of the other groups in that they consisted in stronger flight and avoidance responses, a stronger need for social support, and a more intense mental preoccupation. Increased levels of social tension, lack of social recognition, and isolation indicated chronic stress. Patients with severe alcohol addiction also showed a more pronounced familial predisposition, stronger craving, increased anxiety, a longer history of alcohol abuse, and a higher frequency of incisive life events than patients with mild to moderate alcohol addiction. Conclusions: The results indicate that for a more differentiated evaluation of stress responsiveness and stress coping in persons with an alcohol addiction not only length of abstinence, but also severity of disease have to be taken into account. Further research is necessary to adjust treatment accordingly.

Keywords: alcohol addiction, stress, abstinence duration, disease severity, cortisol, skin conductance, heart rate, TSST. 


\section{Einführung}

Alkohol ist für viele Menschen ein gut schmeckendes und wohltuendes Genussmittel. Man findet ihn bei fast allen gesellschaftlichen Anlässen, er gehört einfach dazu, wenn es fröhlich werden soll, locker und gemütlich. Vereinfacht gesagt ist Alkohol schon längst gesellschaftsfähig geworden und in seiner gesellschaftsbildenden Funktion akzeptiert. Bei vielen Menschen entfaltet Alkohol seine Wirkung wie ein Medikament. Der Trinkende fühlt sich entspannt und beschwingt, sediert und zum Schlafen stimuliert. Andererseits ist der Alkohol aber nicht nur in großen, sondern auch in geringen Mengen toxisch. Insbesondere chronischer Alkoholkonsum kann alle Lebensbereiche betreffen und zu erheblichen körperlichen, psychischen und sozialen Schäden führen. Allerdings entstehen Alkoholmissbrauch und Alkoholabhängigkeit nicht im luftleeren Raum, sondern auf der Basis neurobiologischer Einflüsse sowie im Kontext familiärer Entwicklungsbedingungen, der Alkoholverfügbarkeit und des außerfamiliären sozialen Umfelds. Das alltägliche Leben eines Mitteleuropäers ist in den letzten Jahrzehnten schneller und um ein Vielfaches komplexer geworden. Diese Veränderungen werden mit immer größer empfundener Stressbelastung begleitet. In dem vielschichtigen Prozess der Entstehung und Aufrechterhaltung von Alkoholmissbrauch und Alkoholabhängigkeit, in dem sich die genetische, psychische und soziale Ebene gegenseitig beeinflussen, spielt der Stress, als eine Art Vermittler, eine wichtige Rolle (Schepis et al., 2011; Becker, 2017; Keyes et al., 2011). Die Menschen reagieren auf ein- und denselben Stressreiz unterschiedlich, deshalb ist ihre individuelle Reagibilität auf Umweltreize für den Zusammenhang mit Alkoholmissbrauch- und Abhängigkeit nicht nur für die Medien und die breite Öffentlichkeit von Relevanz, sondern auch für die medizinisch-psychologischen Forschungsbemühungen. Die Annahme, dass Stresserfahrungen zu einer Zunahme 
des Alkoholmissbrauchs führen, gibt es schon lange (Sayette, 1999), aber die zugrundeliegenden Mechanismen sind noch nicht vollständig geklärt (Becker, 2017). In der einschlägigen Literatur finden sich Studien zum Themenbereich der Abhängigkeitsschwere und Stressreagibilität, die sehr heterogene Durchführungsmethoden verwendet haben, mit zum Teil extrem kleinen Stichproben und einem sehr heterogenen Patientenkollektiv. Entsprechend fielen die Ergebnisse sehr unterschiedlich aus und lassen sich kaum generalisieren.

In der eigenen Studie wurde versucht, den Einfluss der Abhängigkeitsschwere und der Abstinenzdauer auf die Stressreagibilität und Stressverarbeitung differenziert herauszuarbeiten (Tolic \& Soyka, 2018). Zunächst werden hier die beiden Phänomene Alkoholabhängigkeit und Stress in ihren wichtigsten Aspekten näher erläutert. Danach wird die eigene publizierte Studie vorgestellt, die Ergebnisse diskutiert und mit anderen empirischen Studien in Beziehung gesetzt. Der publizierte Artikel ist zu finden unter: https://www.thieme-connect.de/products/ejournals/pdf/10.1055/s-0043119798.pdf).

\section{Alkoholabhängigkeit}

\subsection{Begriffsklärung und Diagnostik im Kontext einer zeitlichen Perspektive und} der kategorialen Vielfalt: Alkoholismus, Missbrauch, Abhängigkeit und schädlicher Gebrauch

Die Geschichte des Alkoholkonsums ist fast so alt wie die Menschheit selbst. In der Antike wurde Wein ritualisiert getrunken, verehrt und in manchen Gegenden sogar vergöttert. Im Mittelalter wurde die Wirkungsweise des Alkohols immer differenzierter beschrieben, einerseits in geringen Mengen als Arzneimittel und andererseits in höheren Mengen als Gift (Schott, 2004). Im 19. Jahrhundert kam es zur Bildung von 
Vereinen, die zum Ziel hatten, mehr Bewusstheit, mehr Mäßigung und Enthaltsamkeit im Umgang mit Alkohol zu erreichen. In diesem Zeitrahmen, etwa Mitte des 19. Jahrhunderts, tauchte der Begriff „Alkoholismus“ auf, war sehr unscharf formuliert und umfasste verschiedenste Symptombilder eines problematischen Trinkverhaltens. Erst durch die einflussreichen Arbeiten der Forschergruppe um Edwards und Gross (1976), die Kernsymptome einer Alkoholabhängigkeit von den Folgen des Alkoholkonsums abgrenzten, wurde auch die Trennung zwischen Alkoholmissbrauch und Abhängigkeit vollzogen. Diese Unterscheidung bildete die Grundlage für die Diagnosekriterien der weltweit anerkannten Klassifikationssysteme; die International Classification of Diseases (ICD-10; Deutsche Ausgabe Dilling et al. 1993) und Diagnostisches und Statistisches Manual (DSM-IV, Saß et al., 1996) der American Psychiatric Association. Im Mai 2013 erschien das DSM in seiner 5. Auflage. Heutzutage sind unter dem Alkoholismus vorwiegend Alkoholmissbrauch und Alkoholabhängigkeit gemeint. Allerdings findet man in der ICD-10 statt des Missbrauchsbegriffs Kriterien des schädlichen Gebrauchs (F 10.1). Praktisch wieder ein anderer Begriff, der aber in etwa die gleiche Bedeutung beinhalten sollte wie der Missbrauchsbegriff. Die Kriterien des schädlichen Gebrauchs werden in der ICD-10 wie folgt beschrieben (Auszug):

„Konsum psychotroper Substanzen, der zu Gesundheitsschädigung führt. Diese kann als körperliche Störung auftreten, etwa in Form einer Hepatitis nach Selbstinjektion der Substanz oder als psychische Störung, z. B. als depressive Episode durch massiven Alkoholkonsum“.

Für die Diagnosestellung des schädlichen Gebrauchs nach ICD-10 (F 10.1) ist also eine klare Schädigung der körperlichen oder psychischen Gesundheit erforderlich. Gleichzeitig dürfen die Kriterien einer Alkoholabhängigkeit nicht erfüllt sein. 
Im Grunde genommen beschreiben die Kriterien des schädlichen Gebrauchs kaum das spezifische Verhalten, sondern eher seine Folgen.

Das klinische Bild einer Alkoholabhängigkeit hingegen variiert in der Ausprägung und zeichnet sich durch den Vorrang des Alkoholtrinkens gegenüber anderen Verhaltensweisen sowie durch Fortsetzung des Konsums trotz schädlicher Folgen aus. Die ICD-10 führt folgende 6 Kriterien für das Abhängigkeitssyndrom (hier Alkohol) auf:

- Ein starker Wunsch oder eine Art Zwang, die psychotrope Substanz zu konsumieren;

- Verminderte Kontrollfähigkeit bezüglich des Beginns, der Beendigung und der Menge des Konsums;

- Körperliches Entzugssyndrom bei Beendigung oder Reduktion des Konsums, nachgewiesen durch substanzspezifische Entzugssyndrome oder durch die Aufnahme der gleichen oder einer nahe verwandten Substanz, um Entzugssyndrome zu mildern oder zu vermeiden;

- Nachweis einer Toleranz. Um die ursprünglich durch niedrige Dosen erreichten Wirkungen der Substanz hervorzurufen, sind zunehmend höhere Dosen erforderlich;

- fortschreitende Vernachlässigung anderer Vergnügen oder Interessen zugunsten des Substanzkonsums, erhöhter Zeitaufwand, um die Substanz zu beschaffen, zu konsumieren oder sich von den Folgen zu erholen;

- anhaltender Substanzkonsum trotz des Nachweises eindeutiger schädlicher Folgen, wie z. B. Leberschädigung durch exzessives Trinken, depressive 
Verstimmungen infolge starken Substanzkonsums oder drogenbedingte Verschlechterung kognitiver Funktionen, wobei sich der Konsument über die Art und das Ausmaß der schädlichen Folgen bewusst ist oder davon auszugehen ist.

Die Diagnosestellung erfolgt, wenn irgendwann während der letzten 12 Monate gleichzeitig drei oder mehr der aufgeführten Kriterien vorhanden waren.

In der ersten Auflage des Diagnostischen und Statistischen Manuals Psychischer Störungen, DSM-5 (Deutsche Version; Falkai \& Wittchen, 2015) wurde die Kategorie substanzbezogener Störungen in „Sucht und verwandte Störungen“ umbenannt. Der formale Wechsel von römischen zu arabischen Ziffern (DSM-IV zu DSM-5) kann sicherlich als geringste Veränderung betrachtet werden. Die im DSM-IV geführte Unterscheidung zwischen Missbrauch und Abhängigkeit wurde aufgegeben und als einzige Diagnose die Substanzgebrauchsstörung mit unterschiedlichen Ausprägungsgraden eingeführt. Das hat wahrscheinlich viel größere Auswirkungen für die Diagnostik und die Behandlung substanzbezogener Störungen. Der dimensionale Ansatz ermöglicht das Einbeziehen von Frühstadien einer Alkoholkrankheit. Es gibt praktisch nur noch eine Einstufung nach Schweregrad, wie "leicht“, "mittel“ oder „schwer“. Somit könnten deutlich mehr Menschen mit Behandlungsbedarf erfasst werden. Es wurden folgende diagnostische Kriterien der alkoholbezogenen Störung eingeführt:

- Wiederholter Substanzgebrauch, der zum Versagen bei wichtigen Verpflichtungen in der Schule, bei der Arbeit oder zu Hause führt,

- Wiederholter Substanzgebrauch in Situationen, in denen es Aufgrund des Konsums zu einer körperlichen Gefährdung kommen kann, 
- Fortgesetzter Substanzgebrauch trotz ständiger oder wiederholter sozialer oder zwischenmenschlicher Probleme,

- Toleranzentwicklung charakterisiert durch ausgeprägte Dosissteigerung oder verminderte Wirkung unter derselben Dosis,

- Entzugssymptome oder deren Linderung beziehungsweise Vermeidung durch Substanzkonsum,

- Einnahme der Substanz in größeren Mengen oder länger als geplant,

- Anhaltender Wunsch oder erfolglose Versuche, den Substanzgebrauch zu verringern oder zu kontrollieren,

- Hoher Zeitaufwand für Beschaffung und Konsum der Substanz oder um sich von ihren Wirkungen zu erholen,

- Aufgabe oder Einschränkung wichtiger Aktivitäten aufgrund des Substanzkonsums,

- Fortgesetzter Konsum trotz körperlicher oder psychischer Probleme,

- Starkes Verlangen nach dem Konsum (Craving).

Die Anzahl der erfültten Kriterien bestimmt die Schwere der Störung und ihre Klassifikation (2 bis 3 erfüllte Kriterien: milde Störung, 4 bis 5 Kriterien: moderate Störung, mindestens 6 Kriterien: schwere Störung). Die Aufhebung der kategorialen Unterscheidung in Missbrauch und Abhängigkeit wurde durch empirische Studien begründet, wonach diese unterschiedliche Schweregrade derselben Störung sind. Die Erkenntnisse aus den Studien können gleichzeitig als Vorteil des neuen Diagnosekonzepts gesehen werden. Bemängelt wird allerdings, dass durch die Einbeziehung unscharfer Kriterien des schädlichen Gebrauchs die bis dato in ihren 
wesentlichen Punkten klar beschriebene Diagnose einer Alkoholabhängigkeit weitgehend aufgelöst wird (Maier et al., 2015). Die bevorzugte Suchtdiagnostik ist also eine kategoriale (ICD-10), mit der das Ziel verfolgt wird, das aktuelle Krankheitsbild einer entsprechenden Krankheitskategorie richtig zuzuordnen. Allerdings sind solche Grenzen auf der biologischen Ebene sehr schwierig vorzunehmen. Die Diagnose der Alkoholabhängigkeit umfasst eine sehr inhomogene Patientengruppe, in der sich noch weitere psychische Störungen in Form von Doppeldiagnosen befinden. Die Lösung des Problems der Heterogenität versuchte man in den Siebziger- und Achtzigerjahren durch die Entwicklung von Typologien zu lösen. Alleine für sich stellen aber auch diese keine weiterführende Lösung dar, weil am Ende der diagnostischen Prozesse immer noch zahlreiche Patienten sich nicht zu der jeweiligen Typologie zuordnen lassen. Eine dimensionale Diagnostik sollte vielmehr von einem Einzelfall ausgehen und in einer umfassenden Form nicht nur die Krankheit selbst, sondern die Gesamtheit des Leidens erfassen. Diese berücksichtigt Entstehungsbedingungen der alkoholbezogenen Störung wie die aufrechterhaltenden Faktoren gleichermaßen. Diese können die Störung wesentlich beeinflussen, wie beispielsweise Eigendynamik der Suchtstörung, Stress am Arbeitsplatz oder in der Familie sowie weitere sekundäre Leiden. Jede Erkrankung ummantelt den Betroffenen wie in einer Blase voller unerzählter Erlebnisse, Gedanken, Vorstellungen, Bilder. Die Semipermeabilität dieser Blase wird weitgehend von dem Patienten selbst bestimmt, aber auch zu einem anderen Teil von den ihn umgebenden sozialen Faktoren. Diese kann man sehr schön an dem Umgang der sozialen Umgebung mit dem Leiden des Patienten ablesen. In der praktischen Arbeit kommt es nicht selten vor, dass man einem Patienten begegnet, der stärker an der Stigmatisierung leidet als an der Symptomatik der alkoholbezogenen Störung selbst (Glas et al., 2014). In einem behandlungsorientierten diagnostischen Ansatz können sowohl Symptomgenese- und prozessorientierte als 
auch für den einzelnen Patienten bedeutungsorientierte Elemente vorhanden sein. Unter Einbeziehung von Ressourcen des Patienten lässt sich mit diesem Ansatz eine ausdifferenzierte diagnostische Basis schaffen, die die Erfolgswahrscheinlichkeit einer medikamentösen- und psychotherapeutischen Behandlung erhöht. Das zentrale Ziel besteht somit sowohl in der umfassenden Erfassung des defizitären Verhaltens als auch in der Entdeckung und Nutzung von Ressourcen des Patienten, mit denen eine Veränderung zum abstinenten und eigenständig geführten Leben ermöglicht wird.

\subsubsection{Weitere Begriffe und Definitionen}

Bei der Durchsicht der Literatur findet man, neben den bereits in der ICD-10 und DSMU-5 erläuterten Kategorien, zahlreiche weitere Begriffe im Bereich alkoholbezogener Störungen. Beispielsweise der Begriff des riskanten Alkoholkonsums (hazardous drinking). Bei diesem ist die Wahrscheinlichkeit erhöht, durch exzessiven Alkoholkonsum Schaden zu erleiden. Auf der Basis mehrerer epidemiologischer Studien hat die WHO selbst die Definierung dieses Begriffs vorgeschlagen. Laut Studienergebnissen erhöht sich bei einem täglichen Alkoholkonsum über die von der WHO definierten Grenzen das Risiko für alkoholassoziierte Schäden oder Folgeerkrankungen deutlich (Übersichtsarbeiten bei Rehm et al., 2017, 2016; Rehm et al., 2003; sowie weitere Studien; Burger et al., 2000; Inoué et al., 2001; Edwards, 1997; Seitz et al., 2008). Weitere Begriffe wie exzessives Trinken oder problematisches Trinken werden ebenfalls verwendet, um einen erhöhten und potenziell schädlichen Konsum zu beschreiben. Es gab aber auch Vorschläge, auf den häufig verwendeten Begriff des Alkoholismus zu verzichten (Heather \& Robertson, 1997). 


\section{2 Ätiologie}

Bisher existiert kein einheitliches Störungsmodell für die Entstehung einer Alkoholabhängigkeit. Die eindimensionalen Hypothesen (z. B. Persönlichkeit, Genetik, familiäre Belastung) haben wenig empirische Akzeptanz gefunden, weshalb ein biopsychosoziales Modell geeigneter erscheint, die Entwicklung und Aufrechterhaltung der Alkoholabhängigkeit zu beschreiben (Kruse et al., 2000). Aus dem Zusammenspiel dreier Faktorenkomplexe, nämlich dem biologischen (Genetik), der Eigenwirkung des Alkohols und dem Einfluss psychosozialer Variablen kann man heute im Wesentlichen die Entwicklung einer Alkoholabhängigkeit erklären. Die Ergebnisse zahlreicher Familienuntersuchungen zeigen, dass Söhne von Alkoholikern ein viermal höheres Risiko haben an Alkoholismus zu erkranken als Söhne nicht alkoholkranker Eltern (Schuckit, 2014; Rietschel \& Treutlein, 2013; Schuckit, 2009, 2000; Prescott \& Kendler, 1999; Goodwin, 1992). So wird sowohl in den Adoptionsstudien (z. B. Cloninger et al., 1981; Cadoret et al., 1995) als auch in den Zwillingsstudien (z. B. Prescott \& Kendler, 1999) ein Einfluss genetischer Faktoren von ca. 50 \% angegeben (Schuckit, 2000; True et al., 1999). Für die alkohol- und drogenbezogenen Störungen gemeinsam wird der genetische Einfluss sogar auf $\sim 60 \%$ geschätzt (Schuckit, 2014). Der hohe genetische Anteil scheint sowohl mit dem Beginn als auch mit der Schwere der Alkoholabhängigkeit im Zusammenhang zu stehen (Kapoor et al., 2016; Enoch, 2013; Irwin et al., 1990). Die hier erwähnten Studien dienen lediglich als Beispiele für die zahlreichen wissenschaftlichen Untersuchungen im Bereich des Einflusses der genetischen Prädisposition auf die Entwicklung einer Alkoholabhängigkeit. Das Gleiche gilt für die Studien über die Eigenwirkung des Alkohols als wichtigem Faktor bei der Genese der Alkoholabhängigkeit. Diese Untersuchungen weisen auf eine besondere Gefährdung von denjenigen Menschen, bei denen Alkohol eine entspannende Wirkung erzielt, was 
sich elektroneurophysiologisch nachweisen lässt (z. B. Rangaswamy \& Porjesz, 2014). Nach Schuckit und Mitarbeitern $(1996,2000)$ existiert eine weitere gefährdete Gruppe von Menschen, die akut nur wenig Alkoholwirkung verspüren, sodass ein natürliches Warnsignal fehlt. Welche Gene an der Entwicklung einer erhöhten Alkoholverträglichkeit und somit auch an einer Alkoholabhängigkeit beteiligt sind, ist immer noch nicht endgültig geklärt. Bisher konnten insbesondere die Veränderungen der Regulation an Gamma-Aminobuttersäure-A-Rezeptor Alpha-2 (GABRA2; Soyka et al. 2008; Liebermann et al. 2015), der Genvarianten der Alkoholdehydrogenase 4 (ADH4; Preus et al., 2011) und Genen acetylcholinerger Rezeptoren Muscarin-2 (CHRM2) mit Alkoholismus in Zusammenhang gebracht werden. In einer koreanischen Studie konnte sogar ein Zusammenhang zwischen CHRM2 und der Schwere der Alkoholabhängigkeit gefunden werden (Jung et al., 2011). Der Alkohol entfaltet seine Wirkung an zahlreichen Rezeptoren und steht in Wechselbeziehung mit mehreren Neurotransmittersystemen (Banerjee, 2014). Zu diesen zählen insbesondere opioiderge, serotonerge, gabaerge, glutamaterge sowie dopaminerge Transmittersysteme (Übersicht bei Spanagel, 2009; Tabakoff \& Hoffman, 2013). In einer stark vereinfachten Form kann man sagen, dass Alkohol die Inhibition an GABA-Synapsen verstärkt und gleichzeitig die Exzitation an Glutamatsynapsen reduziert. Außerdem finden Veränderungen am mesolimbischen Belohnungszentrum durch Stimulierung von $\mu$-Opioid-Rezeptoren statt oder es kommt zu einer alkoholiniziierten Ausschüttung von Enkephalin. Alkohol führt sowohl zu einer Erhöhung des Serotoninspiegels als auch zu einer verstärkten Aktivität von Dopamin im mesolimbischen System (z. B. Johnson, 2008). In der Vermittlung von Belohnungseffekten sowohl für die natürlichen Verstärker (Essen, Trinken, Sex) als auch von Alkohol und Drogen spielt das Dopamin als Neurotransmitter eine entscheidende Rolle (Schmidt \& Schaible, 2005). Im Gehirn sind die mesolimbische 
und die mesokortikale Dopaminprojektion sehr verbreitet. Sie umfassen Verbindungen vom ventralen tegmentalen Areal (VTA) zum limbischen System (Amygdala, Hippocampus, Nucleus Accumbens) sowie den präfrontalen, orbitofrontalen und cingulären Kortexregionen (Gardner, 2011). Beim Alkoholkonsum erleben die Menschen subjektiv positive Effekte, die der Alkohol u. a. über einen schnellen Anstieg von Dopamin entfaltet. Chronischer Alkoholmissbrauch, Alkoholabhängigkeit und Entzug sind dagegen mit einer verminderten dopaminergen Aktivität assoziiert (Oswald et al., 2005; Volkow et al., 2003). Diese Effekte bewirken, dass der Reiz, hier Alkohol, durch wiederholte Einnahme aus seinem Kontext hervorgehoben wird und für das Bewusstsein immer zugänglicher wird (Oswald et al., 2005; Volkow et al., 2003). Der wiederholte Konsum führt aber auch zu dauerhaften, teilweise irreversiblen, struktur-synaptischen Veränderungen im Gehirn, die für die kognitiven Funktionen des Lernens und der Gedächtnisbildung bzw. zur Langzeitpotenzierung zuständig sind (z. B. Kalivas \& O'Brien, 2008; Saal et al., 2003). Präklinische Studien weisen auf interagierende Wirkung von Cortisol mit den Belohnungseffekten der abhängigkeitserzeugenden Substanzen hin (Marinelli \& Piazza, 2002; Belujon \& Grace, 2011; Saal et al., 2003). Glukokortikoide und Stress interagieren mit dem dopaminergen Belohnungssystem und erhöhen die Anfälligkeit für die Abhängigkeitsentwicklung (Marinelli \& Piazza, 2002; Oswald et al., 2005).

Die wichtigsten Variablen aus dem psychischen und sozialen Bereich werden hier nur erwähnt. Eine vollständige Berücksichtigung dieser Faktoren würde den Rahmen dieser Arbeit sprengen. Ausführlicher bei Soyka \& Küfner, 2008. Die Fokussierung auf neurobiologische Aspekte der Entstehung einer Alkoholabhängigkeit bedeutet nicht, dass diese einen Hoheitsanspruch gegenüber den psychischen oder sozialen Faktoren haben. Für die Entwicklung einer Alkoholabhängigkeit sind einerseits frühe Lernprozesse und die Vorbilder (häufig sogar elterlicher Alkoholkonsum), andererseits 
die Persönlichkeitsmerkmale wie beispielsweise Affektlabilität, Selbstunsicherheitund die Neigung zu depressiv-dysphorischer Stimmung von Relevanz. Insbesondere der Stress kann als wichtiger Faktor in der Entstehung und Aufrechterhaltung einer Alkoholabhängigkeit angesehen werden (z. B. Stephens \& Wand, 2012; Koob, 2008). In mehreren Studien wird er sogar als einer der bedeutendsten Faktoren bei der Entstehung und Aufrechterhaltung von alkoholbezogenen Störungen angesehen (Schepis et al, 2011; Becker, 2017; Koob et al., 2014; Koob, 2008). Spezifische Faktoren, wie Suchtverlangen, Belohnung, stressige Erlebnisse in der Kindheit, Traumata, Genetik und die Persönlichkeitsmerkmale modulieren die Effekte des stressinduzierten Alkoholmissbrauchs (Breese et al., 2011; Sinha, 2008; Dawson et al., 2005; Thomas et al., 2011). Auch Langzeitstudien belegen, dass verschiedenste Stressoren mit erhöhtem Alkoholmissbrauch in Verbindung stehen (z. B. Keyes et al., 2011). Für weitere Interaktionen zwischen Alkohol und Stress s. Einführung in dem veröffentlichten Artikel (Tolic \& Soyka, 2018). Die Komorbidität mit anderen psychischen Störungen (s. weiter unten) insbesondere Depression, Psychotrauma, Angst, Psychose und ADHS ist hoch (z. B. Preus et al., 2016; Sullivan et al., 2005; Veenstra et al., 2006; Fein, 2015). Hinzu kommen die Persönlichkeitsstörungen, besonders die emotional-instabile, dissoziale sowie ängstlich-vermeidende Persönlichkeitsstörung (Agrawal et al., 2013; Preus et al., 2009). Schließlich existieren in der Umwelt des Betroffenen zahlreiche Konstellationen, die für die Entwicklung von Alkoholabhängigkeit von Bedeutung sind wie z. B.: Herkunftsfamilie, Schule, Gruppen, Konformitätsdruck („Cool-sein“), früh erlerntes Verhalten, Befindlichkeitsstörungen durch Alkohol zu bekämpfen (hier häufig in Folge von Missbrauch, Misshandlung und Vernachlässigung in der Kindheit, aber auch eigene psychopathologische Auffälligkeiten im Kindes- und Jugendalter wie Ängste, Unsicherheiten, Depression, Integrationsschwierigkeiten, nicht gemeisterte 
Entwicklungsaufgaben, inneffektive Bewältigungsversuche, Arbeitssituation, finanzielle Situation, Verfügbarkeit von Alkohol, Preise, Medien u. v. a. m.

\subsection{Epidemiologie}

Risikofaktor Alkohol

Ein regelmäßiger, dauerhafter und in hohen Mengen praktizierter Alkoholkonsum ist mit zahlreichen körperlichen und psychischen Schäden assoziiert. Aus epidemiologischer Sicht zählt Alkoholmissbrauch zu den relevantesten Risikofaktoren, deren Kosten-Nutzen-Bilanz negativ ist (z.B. Küfner \& Kraus, 2002; Klingemann \& Gmel, 2001). Alkoholabhängigkeit ist eine chronisch-rezidivierende Erkrankung, die zu den häufigsten psychischen Störungen gehört. Nach Schätzungen der Deutschen Hauptstelle für Suchtfragen e. V. (DHS) sind 3,38 Millionen Menschen in Deutschland in den letzten zwölf Monaten von einer Alkoholgebrauchsstörung betroffen gewesen (Missbrauch 1,61 Mio.; Abhängigkeit 1,77 Mio. DHS, Jahrbuch Sucht, 2018). Der Gesamtverbrauch an alkoholischen Getränken im Jahr 2017 betrug 133,8 Liter pro Kopf der Bevölkerung. Der Durchschnittskonsum der Mehrheitsbevölkerung (15-65jährige) beträgt 14 Liter Reinalkohol. Allein im Jahr 2016 wurden in den Krankenhäusern 322.608 Behandlungsfälle mit einer psychischen oder verhaltensbezogenen Störung durch Alkohol diagnostiziert, was als zweithäufigste Einzeldiagnose in der Krankenhausstatistik geführt wird (DHS, Jahrbuch Sucht 2018). Diese Diagnose lag bei den Männern sogar auf dem ersten Platz der Hauptdiagnosen. Etwa 74.000 Todesfälle pro Jahr werden durch Alkoholkonsum oder den kombinierten Konsum von Alkohol und Tabak verursacht. Die DHS gibt an, dass die direkten und indirekten Kosten des Alkoholkonsums in Deutschland auf ca. 40 Mrd. Euro beziffert werden. Demgegenüber stehen etwa 3,17 Mrd. Euro staatlicher Einnahmen aus alkoholbezogenen Steuern. Die hohe Anzahl an Betroffenen mit entsprechenden 
Folgekosten verdeutlicht, neben der Notwendigkeit der genaueren Erforschung von Entstehungsbedingungen und der Entwicklung differenzierter Diagnostik, auch den Bedarf an stärker individualisierten effektiven Therapien (Mann, 2010).

\subsection{Somatische und psychische Folgeschäden des Alkoholismus}

Wie aus den epidemiologischen Daten ersichtlich, gehen alkoholbezogene Störungen mit erheblichen körperlichen, psychischen und sozialen Folgen für den Betroffenen, seine Familie und die Gesellschaft einher. Alkoholmissbrauch und Abhängigkeit können zu einem ganzen Spektrum organischer Schäden und syndromaler Beeinträchtigungen führen (ausführlicher bei Rehm et al., 2017; Singer \& Teyssen, 2005). Fast jedes Organ kann in Folge eines Alkoholmissbrauchs akut (z. B. Folgen einer Intoxikation) oder chronisch (z. B. dekompensierte Leberzirrhose) geschädigt werden. Als Beispiel wird hier aus methodischen Gründen und im Zusammenhang mit der durchgeführten Studie (Ausschlusskriterium) der Kater („alcoholic hangover“, wissenschaftlich „Veisalgie“) als Folge einer Alkoholintoxikation kurz vorgestellt. In den ersten 24 Stunden nach Alkoholkonsum bestehen bei Alkoholabhängigen noch zahlreiche pathophysiologische Erscheinungen, weshalb aus methodischen Gründen keine objektivierbaren Untersuchungen zur Stressreaktivität durchführbar sind. Beim alkoholinduzierten Kater handelt es sich um den meist vorkommenden Symptomkomplex eines exzessiven Alkoholkonsums (Verster et al., 2010). Nach einem Alkoholexzess können zahlreiche Symptome auftreten, die für den Betroffenen unangenehm sind. Zu den drei häufigsten Symptomen zählen Kopfschmerzen, Durst und Müdigkeit. Slutske und Mitarbeiter konnten in einer großen epidemiologischen Untersuchung an 1200 nicht abstinenten Collegestudenten Katersymptome und ihre Frequenz erfassen (Slutske et al., 2003). Der Häufigkeit nach und neben bereits erwähnten Symptomen wurden folgende Katersymptome erfasst: trockener Mund, 
Niedergeschlagenheit, Übelkeit und Erbrechen, Konzentrationsstörungen, Licht- und Geräuschempfindlichkeit, vermehrte Schweißneigung, Magen- und Muskelschmerzen, depressive Verstimmung, Reizbarkeit, schneller Pulsschlag, Tremor, Durchfall, gerötete und trockene Augen. (Übersicht bei Verster et al., 2010). Meistens treten die Symptome Stunden nach Ende des Alkoholkonsums ein, wobei der Alkohol zu dem Zeitpunkt oft vollständig eliminiert ist (Verster et al., 2010). Nach seiner Aufnahme über Magen- und Dünndarmschleimhäute wird der Alkohol vorwiegend in der Leber über das Enzym Alkoholdehydrogenase zu Azetaldehyd abgebaut. Die toxischen Effekte des Azetaldehyds werden sowohl für die typischen Katersymptome als auch für die zahlreichen Organschäden einschließlich Tumorentwicklung bei Alkoholabhängigkeit verantwortlich gemacht (Guo \& Ren, 2010; Seitz \& Becker, 2007).

Andere vom chronischen Alkoholmissbrauch betroffenen Organe und Organsysteme unterscheiden sich stark hinsichtlich des Ausmaßes der alkoholassoziierten Organschäden. Die genauen Ursachen für die interindividuellen Unterschiede sind noch nicht geklärt, ebenso wie die Tatsache, dass nicht jede Person, die Alkohol konsumiert, auch erkrankt. Der Bereich der alkoholassoziierten Organschäden ist sehr umfangreich, weshalb auf entsprechende ausführlichere Literatur verwiesen wird (Rehm et al., 2017; Singer \& Teyssen, 2005). Sowohl Alkohol als auch Stress entfalten ihre Wirkungen im endokrinen System. Deswegen und auch wegen der komplexen Wechselwirkungen zwischen den beiden wird dieses System gesondert und etwas ausführlicher vorgestellt (s. u.). In einem ebenfalls bedeutsamen Ausmaß existieren zahlreiche psychische und soziale Folgen des chronischen Alkoholmissbrauchs. Diese reichen von Auswirkungen auf die Arbeitsfähigkeit und die Stressbewältigung des Betroffenen, seine Gedächtnisleistungen bis hin zu schwerwiegenden Folgen für die Familie und die Gesellschaft. Insbesondere schwerkranke Alkoholabhängige zeigen 
erhöhte Werte in den Bereichen Mangel an sozialer Anerkennung, soziale Spannungen und Isolation (Tolic \& Soyka, 2018). Diese Werte stehen als Zeichen starker und chronischer Stressbelastung. Ausführlicher über psychische und soziale Folgen des Alkoholismus z. B. bei Soyka \& Küfner, 2008. Kap.5.

\subsection{Alkoholismus und Auswirkungen auf das endokrine System}

Zusammen mit dem Nervensystem sorgt das endokrine System für die lebensnotwendige Kommunikation zwischen den Organen im Körper und für die Aufrechterhaltung der Homöostase. Fast jedes Organ und jede Zelle im Organismus wird vom endokrinen System beeinflusst. Sowohl moderater als auch chronischer Alkoholmissbrauch kann unterschiedliche Auswirkungen auf das Endokrinium haben. Alkoholmissbrauch beeinflusst verschiedene Hormonsysteme wie die HypothalamusHypophysen-Nebennierenrinden-Achse (HHNA), die Hypothalamus-HypophysenGonaden-Achse (HHGA), die Hypothalamus-Hypophysen-Schilddrüsen-Achse (HHSA) und die Hypothalamus-Hypophysen-Wachstumshormon-Achse (HHWA). Außerdem werden andere Hormone wie beispielsweise Prolaktin und die Bauchspeicheldrüse (Pankreas) durch Alkoholmissbrauch beeinflusst.

\subsubsection{Alkohol und Hypothalamus-Hypophysen-Nebennierenrinden-Achse (HHNA)}

Zahlreiche Effekte der akuten Alkoholwirkung stehen mit hypothalamischen, aber auch mit extrahypothalamischen Vorgängen der Stressregulation in Verbindung. Eine Blutalkoholkonzentration von 0,8 \%o geht mit einer hypothalamischen Aktivierung und Erhöhung des Cortisolspiegels, des adrenokortikotropen Hormons (ACTH) und des Norepinephrin einher (Magrys et al., 2013; Allen et al., 2011). Akuter Alkoholkonsum aktiviert also die HHNA, was zu einer dosisabhängigen Erhöhung des zirkulierenden ACTH, Cortisols sowie der Glukokortikoiden führt (Richardson et al., 2008). Allerdings unterliegen die Effekte interindividuellen Unterschieden. So zeigen 
beispielsweise Alkoholabhängige mit einer positiven familiären Alkoholanamnese eine Hyperreaktivität der HHNA (Söderpalm et al., 2011; Uhart et al., 2006; Zimmermann et al., 2004). In der Trinkphase zeigen Alkoholabhängige eine erhöhte Aktivierung der HHNA, was sich in einem Tagesprofil erhöhter Cortisolwerte zeigt, ebenso bei einem Alkoholrückfall (Wand \& Dobs, 1991; Adinoff et al., 2003; Junghanns et al., 2003; 2005). In der frühen Abstinenzphase hingegen zeigen Alkoholabhängige eine abgeschwächte Aktivität der HHNA, die sich bei einem experimentellen Stressparadigma ebenfalls zeigt (Stephens \& Wand, 2012; Lovallo et al., 2000; Tolic \& Soyka, 2018). Eine hochgradige Hyperaktivität der kortikostriatalen Regionen, insbesondere des ventromedialen präfrontalen Kortex (VmPFC) und des ventralen Striatums (VS) konnten bei Alkoholikern in der frühen Abstinenzphase gefunden werden (Blaine \& Sinha, 2017). Die abgeschwächte HHNA-Aktivität kann bei Alkoholabhängigen monatelang andauern und gilt in bestimmten Fällen als Prädiktor für Alkoholrückfälle (z. B. Adinoff et al., 2005, Junghanns et al., 2005). Die HHNA-Aktivität spielt nicht nur bei einem Alkoholrückfall, sondern auch bei der Entstehung von Alkoholverlangen eine wichtige Rolle (Blaine et al., 2016; Sinha, 2011; Fox et al., 2007; Sinha et al., 2009) und wird mit Rückfällen bei abstinent lebenden Alkoholabhängigen in Zusammenhang gebracht (Sinha, 2011; Adinoff et al., 2005; Breese et al, 2005; Junghanns et al., 2003). Alkoholabhängige greifen in solchen Situationen $\mathrm{zu}$ unterschiedlichen maladaptiven Stressbewältigungsstrategien (Mattoo et al., 2009). Auch neuere Studien weisen darauf hin, dass dysfunktionale Veränderungen der HHNA mit dem Schweregrad der Abhängigkeit im Zusammenhang stehen (Blaine \& Sinha, 2017). Zwischen Suchtverlangen und der Höhe des Plasmacortisols konnte ein negativer Zusammenhang gefunden werden (Blaine \& Sinha, 2017; O'Malley et al., 2002). Diese Tatsache konnte im pharmakotherapeutischen Bereich genutzt werden, indem 
mittels des Opiatantagonisten Naltrexon und gleichzeitig erhöhten ACTH- und Cortisolspiegeln das Rückfallrisiko reduziert wird (Kiefer et al., 2006). In einer sehr anschaulichen Übersichtsarbeit und Metaanalyse konnte, wenn auch knapp, für das Acamprosat (Campral®) und für Naltrexon eine signifikante Wirksamkeit in der Aufrechterhaltung der Abstinenz nachgewiesen werden (Jonas et al., 2014). Für Disulfiram (Antabus $\AA^{\circ}$ ) konnte hingegen keine signifikante Wirksamkeit gefunden werden. Eine Normalisierung der HHNA erfolgt nach einer Abstinenzdauer von ca. 12 Wochen (Ehrenreich et al., 1997). Eine andere Studie geht von einer 8-wöchigen Alkoholabstinenz aus (Coiro et al., 2007).

Über längerfristige Auswirkungen des Alkohols auf die HHNA ist leider wenig bekannt. In einer Studie wurden Alkoholabhängige mit einer durchschnittlichen Abstinenz von 22 Tagen und noch einmal mit 117-tägiger Abstinenz untersucht. Nach 117 Tagen zeigten die Probanden eine stärkere Cortisolaufwachreaktion, was als potenzieller Hinweis für veränderte HHNA-Aktivität bewertet wurde (Junghanns et al., 2007). In einer anderen Studie wurden Alkoholiker, die durchschnittlich 3,5 Jahre abstinent lebten, mit "Sozialtrinkern“, die als Kontrollprobanden dienten, in einem experimentellen Laborstresstest verglichen. Es konnten keine Unterschiede in der ACTH und HHNA-Stressantwort zwischen den Stichproben gefunden werden (Munro et al., 2005). Die erwähnten Studien haben aber sehr heterogene Ergebnisse erzielt. Häufig hatten sie zum Ziel, lediglich die Zusammenhänge zwischen Stress und Alkoholverlangen zu untersuchen. Die unterschiedlichen Ergebnisse überraschen nicht, wenn man genau analysiert, wie groß die methodischen Unterschiede, insbesondere bei der Stressinduktion, sind. 
Insgesamt aber konnten pathogenetische Auswirkungen des Alkoholismus auf die HHNA-Aktivität in zahlreichen Studien nachgewiesen werden. Eine ausführlichere Darstellung über Auswirkungen des Alkoholmissbrauchs auf die HHNA findet sich im veröffentlichten Artikel (Tolic \& Soyka, 2018) sowie bei mehreren Übersichtsarbeiten (z. B. Blaine \& Sinha, 2017; Stephens et al., 2014; Stephens \& Wand, 2012). Ausführliche Darstellung der HHNA und ihre Funktion s. weiter unten im Kapitel Stress.

Alkohol und andere Hormonsysteme

Die pathologischen Wirkungen von Alkohol auf HHSA wurden durch klinische Studien ebenfalls bestätigt. Ein momentaner Alkoholkonsum hat kaum Auswirkungen auf die Schilddrüsenfunktion. Bei Patienten mit chronischem Alkoholmissbrauch konnte eine abgeschwächte Antwort des Thyreo-stimulierenden Hormons (TSH) auf das Thyreotropin-releasing-Hormon (TRH) gefunden werden (Pienaar et al., 1995). Die abgeschwächte Antwort stand auch mit der Schwere der Entzugssymptomatik im Zusammenhang. Die Ergebnisse bezüglich der peripheren Schilddrüsenhormone T3 und T4 sind widersprüchlich. Ein chronischer Alkoholmissbrauch kann eine Dysfunktion der HHGA verursachen. Im Akutstadium führt Alkoholmissbrauch zu einer Erhöhung von FSH bei Frauen als auch zur Verringerung von Testosteronspiegeln bei Männern (Übersicht bei Rachdaoui \& Sarkar, 2013). Chronischer Alkoholmissbrauch führt häufig bei beiden Geschlechtern zu schweren Dysfunktionen der HHGA, die Fertilitätsstörungen, irregulären Menstruationszyklus, Hypogonadismus, und sexuelle Funktionsstörungen umfassen (Rachdaoui \& Sarkar, 2013). Akuter und chronischer Alkoholismus hat schließlich eine schädliche Wirkung auf die HHWA. Beide Formen des Alkoholismus führen zu einer verminderten Freisetzung von Wachstumshormonen (Rachdaoui \& Sarkar, 2013). 


\subsection{Alkoholabhängigkeit und multipler Substanzgebrauch}

\section{(Mehrfachabhängigkeit)}

Die Komorbidität mit anderen substanzbezogenen Störungen wie Drogen- oder Nikotinabhängigkeit ist häufig. Alkohol- und Tabakabhängigkeit sind die häufigsten substanzbezogenen Störungen. Ihr Konsum und die daraus entstehenden Folgeschäden werden bei weitem permissiver betrachtet als diejenigen von illegalen Drogen. Der Tabakkonsum, insbesondere ein früh beginnender, ebnet nach Meinung vieler Experten den Weg für einen späteren Alkohol- und Drogenmissbrauch. Die Prävalenz von Nikotinabhängigkeit unter Alkoholabhängigen ist sehr hoch und steht im Zusammenhang mit der Trinkmenge und dem Schweregrad der Alkoholabhängigkeit (Grant et al., 2004a). Es existieren Anhaltspunkte über genetische Gemeinsamkeiten in der Entwicklung von Nikotin- und Alkoholabhängigkeit. Sowohl einige Humanstudien (True, et al., 1999) als auch experimentelle Tieruntersuchungen brachten wertvolle Ergebnisse (Crabbe \& Lovinger, 2005). Eine große Mehrheit der Alkohol- und Drogenabhängigen kann nur schwer längerfristig nikotinabstinent leben. Sie sind meist stärker nikotinabhängig und zeigen höhere Serumnikotinkonzentrationen als andere Raucher (z. B. Hurt et al., 1995). Die Mehrfachabhängigkeit von unterschiedlichen Substanzgruppen kann als Folge einer generellen psychischen und/oder biologischen Anfälligkeit betrachtet werden. Entsprechend konnten Prädiktoren für die Abhängigkeitsentwicklung sowohl im psychosozialen als auch biologischen Bereich gefunden werden (s. Kap. 2.2 Ätiologie). Hierbei sind die Lernprozesse auf der Basis einer klassischen oder operanten Konditionierung zu erwähnen, die das Suchtverhalten deutlich löschungsresistent machen. Diese Prozesse beinhalten gewohnte Konsumierung mehrerer Substanzen, Summierung der erwünschten Effekte und den Abbau 
unerwünschter Nebenwirkungen der einen Substanz durch die Einnahme einer anderen. Viele Erklärungsmodelle beziehen sich auf die gleichartige Beeinflussung des „Belohnungssystems“ im Gehirn. Alkohol, Opiate, Amphetamine, Kokain und Nikotin führen sowohl direkt als auch indirekt zur dopaminergen Stimulierung im mesokortikolimbischen System (z. B. Koob \& Volkow, 2010; Koob et al., 2014; Dani \& Harris, 2005). Insgesamt werden durch diese Befunde multiple Gemeinsamkeiten, Wechselwirkungen und synergetische Effekte bei Mehrfachabhängigkeiten deutlich.

\subsection{Alkoholabhängigkeit und Komorbidität mit psychischen Störungen ohne}

\section{Substanzgebrauch}

Im klinischen Alltag begegnet man häufig komplexen Situationen, wenn zu einer bestehenden Alkoholabhängigkeit eine weitere schwere Erkrankung hinzukommt wie beispielsweise eine Posttraumatische Belastungsstörung (PTBS), Psychose, Borderline-Persönlichkeitsstörung, Depression oder Suizidalität. Über die Gründe für die Komorbiditäten kann man in verschiedene Richtungen und auf unterschiedlichen somatopsychischen Ebenen diskutieren. Ein Grund kann darin bestehen, dass der Patient durch die Abhängigkeitserkrankung sehr belastet ist. Ein anderer Grund kann in der alkoholinduzierten Veränderung von denjenigen Hormonellen- und Neurotransmittersystemen liegen, die auch in der Genese anderer psychischer Störungen eine wichtige Rolle spielen (Ho et al., 2011; Kienast \& Heinz, 2006; Kienast et al., 2008; Heinz et al., 2011). Liegt zu einer bestehenden Alkoholabhängigkeit eine weitere psychische Störung vor, spricht man aus psychodiagnostischer Sicht von einer Doppeldiagnose. In der klinischen Praxis deuten Doppeldiagnosen häufig auf die besondere Komplexität der Therapie hin. Zuverlässige Studien mit repräsentativen klinischen Stichproben geben an, dass über $30 \%$ der Patienten mit einem potenziell schädlichen Alkoholkonsum in ihrem Lebenslauf mindestens eine weitere psychische 
Störung erleiden (z. B. Lieb \& Isensee, 2002). Umgekehrt entsteht bei zahlreichen Patienten mit anderen psychischen Störungen eine sekundäre Alkoholabhängigkeit, häufig im Rahmen einer Symptomentlastung. Epidemiologische Untersuchungen haben ein zweifach erhöhtes Risiko für Angststörungen bei Menschen mit Alkoholabhängigkeit im Vergleich zur Allgemeinbevölkerung nachgewiesen (Agosti \& Levin, 2006). So zeigen beispielsweise Menschen mit einer PTBS im Vergleich mit der Allgemeinbevölkerung eine wesentlich höhere Wahrscheinlichkeit für stoffgebundene Abhängigkeitserkrankungen (Übersicht bei Schäfer \& Najavits, 2007). Speziell zwischen PTBS und alkoholbezogenen Störungen wird die Komorbidität auf $15 \%$ geschätzt (Driessen et al., 2008) und werden mit einem höheren Grad an Krankheitsschwere in Verbindung gebracht (McCarthy \& Petrakis, 2010). Die mehrfach bestätigten Zusammenhänge zwischen PTBS und Alkoholismus (Debell et al., 2014) sind vielschichtig und konzentrieren sich sowohl auf die neurobiologische als auch auf die verhaltenspsychologische Ebene der Komorbidität und des Stressgeschehens (aktuelle Übersicht z. B. Gilpin \& Weiner, 2017). Die hohe Zahl an PTBS-Diagnosen bei alkoholabhängigen Patienten wird, wie bei anderen psychischen Störungen, auf multikausale Ursachenmodelle zurückgeführt. Die beim Trauma immer wieder entstehenden negativen Emotionen werden mit verstärktem Verlangen nach Alkohol in Zusammenhang gebracht (Coffey et al., 2006). Ein wesentlicher Grund besteht in der großen Anzahl und Frequenz von frühen Traumatisierungen, vor allem in Form von sexueller oder physischer Gewalt (Übersicht bei Simpson \& Miller, 2002; Schäfer et al., 2017). Die traumatischen Symptome können dann auf den oben genannten Ebenen sehr früh die Fähigkeit zur Stressregulation erheblich schwächen und damit die Entwicklung einer Alkoholabhängigkeit begünstigen (Enoch, 2011). Schwerkranke Alkoholabhängige weisen eine höhere Anzahl einschneidender Lebensereignisse als auch eine verlängerte neuroendokrine Stressreaktion nach Stressexposition auf 
(Tolic \& Soyka, 2018). Der Nachweis existiert wohl schon längst, dass reduzierte Emotionsregulationsfähigkeit mit der Entwicklung stoffgebundener Abhängigkeiten in Verbindung steht (Übersicht bei Aldao et al., 2010). Im Verlauf einer Alkoholabhängigkeit entwickeln sich neben PTBS noch häufiger die depressiven Störungen (King et al., 2003). So konnte in einer repräsentativen Studie herausgefunden werden, dass $40,7 \%$ der Personen mit problematischem Alkoholkonsum zusätzlich mindestens eine Störung aus dem affektiven Formenkreis aufweisen, die unabhängig vom Substanzkonsum entstand (Grant et al., 2004b). Menschen mit Alkoholabhängigkeit zeigen eine zweifach erhöhte Prävalenz für Störungen aus dem depressiven Formenkreis im Vergleich zur Allgemeinbevölkerung. Die Studien zeigen allerdings relativ große Unterschiede in der Studienmethodik wie beispielsweise bei eingesetzten Erhebungsinstrumenten, was sich darauffolgend in den unterschiedlichen Prävalenzraten niederschlägt (z. B. Soyka \& Lieb, 2004; Lai et al., 2015). Ein Alkoholentzug kann beispielsweise Symptome verursachen, die einer psychischen Störung gleichen, diese bilden sich aber innerhalb von ein paar Wochen wieder zurück (Übersichtsarbeiten bei Boden \& Fergusson, 2011; Schuckit, 2006; Conner et al., 2009). Evidenzbasierte Behandlungsprogramme zur Behandlung von alkoholbezogenen Störungen und Depression existieren schon länger, sollten allerdings in Form integrativer Behandlungsansätze auch erfolgreich implementiert werden (McGovern et al., 2010). Nicht weniger komplex gestaltet sich eine Behandlung Alkoholabhängiger mit komorbider Borderline-Persönlichkeitsstörung. Im klinischen Alltag jedenfalls kommt diese Komorbidität häufig vor. Mit $57 \%$ geben Studien hohe Prävalenzraten für Persönlichkeitsstörungen bei Alkoholgebrauchsstörungen generell an und speziell für die Borderline-PS $13 \%$ (Zikos et al., 2010). Die Komorbidität substanzbezogener Störungen mit Persönlichkeitsstörungen wird mit $60 \%$ ohnehin als sehr hoch eingeschätzt (Zanarini 
et al., 2004). Bei solchen Patienten zeigt sich im klinischen Alltag die ganze Summe des Ineinandergreifens der dysfunktionalen Störungsmuster. Neben der Schwierigkeit mit der Einhaltung von Regeln sowie der allgemein eingeschränkten Selbstkontrolle stellt die hohe Suizidrate (Preuss et al., 2006; Driessen, 2004) bei dieser Patientengruppe ebenfalls ein großes Problem dar (Übersichtsarbeit bei Wilcox et al., 2004). Nicht weniger schwierig gestaltet sich die Behandlung von Alkoholabhängigen mit einer komorbiden Aufmerksamkeitsdefizit-Hyperaktivitätsstörung (ADHS). Mit einer Prävalenz zwischen $3 \%$ und $5 \%$ zählt die Störung zu den häufigen psychischen Störungen und zeichnet sich durch eine hohe Persistenz (50-60\%) bis ins Erwachsenenalter aus (Faraone et al., 2006; Biedermann et al., 2000). Für das Störungsbild generell ist die hohe Komorbiditätsrate von bis zu $50 \%$ kennzeichnend (Sullivan \& Rudnik-Levin, 2001). Diese enorm hohe Komorbiditätsrate ist sowohl für den Schweregrad als auch für die Chronizität des Krankheitsverlaufs von hoher Bedeutung. Zu den häufigsten komorbiden Störungen bei ADHS zählen beispielsweise Alkoholmissbrauch, Angststörungen, Depression und Störungen des Sozialverhaltens. Ein früher Beginn des Alkoholmissbrauchs wird ebenfalls häufig beobachtet, genauso wie ein Multisubstanzkonsum (Gillberg et al., 2004; Molina et al., 2000). Ein früher Beginn gilt als wesentlicher Prädiktor für erhöhten Alkoholkonsum (Ellickson et al., 2001). Bei gleichzeitigem Vorliegen von beiden Störungen erhöht sich das Risiko für die Entwicklung weiterer psychischer Störungen deutlich, v. a. für Angst- und depressive Störungen (Willens et al., 2005). Auch höhere Schweregrade des Alkoholund Drogenmissbrauchs sowie schlechtere Behandlungsergebnisse werden bei gleichzeitigem Vorliegen einer ADHS beobachtet (Biedermann et al., 1998).

Bei Störungen mit Substanzabhängigkeit ist das Suizidrisiko stark erhöht (Harwood et al., 2006; Schneider et al., 2006). Das Suizidrisiko ist bei beiden Geschlechtern mit Alkoholismus signifikant erhöht und insbesondere bei Alkoholabhängigen im mittleren 
Lebensalter sehr hoch (Schneider et al., 2009). Bei Alkoholabhängigen beträgt das Lebenszeitsuizidrisiko etwa 8 \% und bleibt über die Lebenszeit konstant (Inskip et al., 1998). Das Suizidrisiko ist besonders dann erhöht, wenn eine Komorbidität mit affektiven Störungen vorliegt (Schneider et al., 2006). Bei stationär psychiatrischen Patienten erhöhten insbesondere Persönlichkeitsstörungen und die Depression das Suizidrisiko. Betrachtet man die Ergebnisse im Bereich der Suizidversuche, so ergeben sich ebenfalls klar erhöhte Risikowerte bei Alkoholkranken. Die Studien zeigen, dass Depression, Stress sowie vorangegangene Suizidversuche die relevantesten Risikofaktoren für suizidales Verhalten bei Alkoholabhängigen darstellen (Preus et al., 2003; Yaldizli et al., 2010; Kelly et al., 2001). Alkoholabhängige mit suizidalem Verhalten in der Anamnese weisen höhere Werte für impulsives und aggressives Verhalten auf (Koller et al., 2002). Betrachtet man die aktuelle Studienlage zur Komorbidität von Alkoholismus und psychotischen Störungen, so zeigen die Untersuchungsergebnisse ein häufiges gemeinsames Vorkommen der beiden Störungen auf. In einer deutschen klinisch-epidemiologischen Studie an 2337 Patienten mit Schizophrenie fanden die Forscher eine Lebenszeitprävalenz von $29,4 \%$ für Abhängigkeitserkrankungen (Schnell et al., 2010). Die höchsten Komorbiditätsraten von ca. $40 \%$ wiesen stationäre Patienten auf.

Wie oben schon erwähnt, gilt die Behandlung von Patienten mit Doppeldiagnosen als schwierig, so dass manche Autoren sogar von einer "mission impossible" sprechen (Chow et al., 2012). 


\subsection{Schwere der Alkoholabhängigkeit}

Bei der Durchsicht wissenschaftlicher Literatur zum Thema Abhängigkeitsschwere findet man zahlreiche Modellvorschläge und Typisierungskonstrukte, wie eine Schweregradeinschätzung erfolgen könnte (z. B. Babor et al.,1992, Cloninger et al., 1981). Allerdings erfolgt die Auswahl der Kriterien sehr uneinheitlich, was eine Definierung des Begriffes erschwert. Wie bereits bei der Diagnostik erwähnt, stellen die Forschungsarbeiten von Edwards und Gross (1976) die wichtigste Basis sowohl für die internationalen Klassifikationssysteme ICD-10 und DSM-5 als auch für die Erfassung der Schwere einer Alkoholabhängigkeit dar. Sie haben erstmals das Konstrukt des Alkoholabhängigkeitssyndroms ausführlich beschrieben und die Kernsymptomatik von den Folgestörungen des Konsums getrennt dargestellt. Die beiden Autoren erwähnten die Variabilität der Kernsymptomatik hinsichtlich des Auftretens der Symptome (vorhanden/nicht vorhanden) und der Intensität der Ausprägung. Hier wird klar eine Komponente des Schweregrads der Symptomatik angesprochen im Sinne ,je mehr und je intensiver die Symptome, desto höher der Schweregrad“" Die ICD-10 berücksichtigt für die Diagnosestellung keine Schweregradeinteilung. Im Gegensatz zur ICD-10 ist im DSM-5 weitgehend eine Schweregradeinteilung bei der diagnostischen Einschätzung vorgesehen. Man könnte fast konstatieren, dass wir mit dem DSM-5 diagnostisch-forschungsmäßig wieder dort sind, wo wir schon 1976 waren. Die Forschungsarbeiten der beiden Autoren legten aber auch einen Grundstein für die Entwicklung verschiedener psychologischer Testverfahren. Einige wurden auf der Basis des Abhängigkeitssyndroms aufgebaut. Einige weitere Fragebögen wurden später aber auch auf der Basis weiterer theoretischer Konstrukte aufgebaut und dienten vorwiegend der Diagnosestellung und der Messung des Schweregrades der Alkoholabhängigkeit. An dieser Stelle werden 
die wichtigsten standardisierten Testverfahren, die zur Beurteilung des Schweregrades der Alkoholabhängigkeit dienen, kurz vorgestellt.

\subsubsection{European Addiction Severity Index (EuropASI)}

Beim EuropASI (Gsellhofer et al., 1993) handelt es sich um ein multidimensionales und halbstrukturiertes Interview, das häufig sowohl im klinischen als auch im Forschungsbereich für Diagnostik und Evaluation substanzbezogener Störungen eingesetzt wird. Die deutsche Version wurde nach der amerikanischen Originalversion (McLellan et al., 1980) entwickelt und erfasst insgesamt sieben Lebensbereiche eines Betroffenen. Es besteht aus 150 Items, mit denen Infos aus den Problembereichen körperlicher Zustand, Arbeits- und Unterhaltssituation, Alkoholgebrauch, Drogengebrauch, rechtliche Situation, Familien- und soziale Beziehungen und psychischer Status erfragt werden. Die Schweregrad-Ratings werden für jeden Bereich getrennt auf einer Skala beurteilt und ein Schweregrad-Profil erstellt. Die Skala reicht von 0 (keine Behandlung erforderlich) bis 9 (Behandlung absolut erforderlich). Die Ratings dienen als Norm dafür, inwieweit eine adäquate Behandlung erforderlich ist. Die deutsche Version des EuropASI wurde auf der Basis von drei Stichprobenuntersuchungen entwickelt. EuropASI wird als geeignetes Instrument für die Zuordnung von Patienten zu adäquaten Therapiesettings und zur Bestimmung des Schweregrads der Alkoholabhängigkeit angesehen (Schmidt et al., 2007). Aus diesem Grund wurde EuropASI in der eigenen Studie eingesetzt.

\subsubsection{Skala zur Erfassung der Schwere der Alkoholabhängigkeit (SESA)}

Die SESA wurde 1992 von Ulrich John und Mitarbeitern als Papier-BleistiftFragebogen entwickelt (John et al., 2001). Das Ziel war, die Kernmerkmale des Abhängigkeitssyndroms auf der Basis von Selbstaussagen der Probanden zu erfassen. Sie wurde auf der Basis von drei Studien mit insgesamt 774 Probanden 
entwickelt und erfüllt die meisten Kriterien des Alkoholabhängigkeitssyndroms nach Edwards und Gross (1976). Die SESA besteht aus sieben Subskalen, und zwar: Einengung des Trinkverhaltens (E), körperliche Entzugssymptome (K), Vermeidung von Entzugssymptomen (V), psychische Entzugssymptome (Verlangen) (P), Toleranzsteigerung $(\mathrm{T})$, extreme Toleranzsteigerung (Te) und Toleranzumkehr (Tu). Bei der Auswertung werden die Rohwerte der einzelnen Subskalen in gewichtete Werte umgewandelt. Diese werden wiederum in Prozentränge oder T-Werte umgewandelt und es wird ein Gesamttestwert ermittelt. In der klinischen Praxis lässt sich der Gesamtscore grob in eine leichte, mittlere und hohe Ausprägung der Abhängigkeit einteilen und bewerten. Konkret werden Prozentränge bis 25 als leichte, 25-75 als mittlere und über 75 als schwere Abhängigkeit interpretiert. Schließlich lassen sich die Prozentränge der einzelnen Subskalen in einem Ausprägungsprofil abbilden. Die Durchführung ist relativ einfach und ökonomisch. Vor der Durchführung sollte aber die Diagnose der Alkoholabhängigkeit durch ICD-Kriterien feststehen. Neben EuropASI wurde SESA in der eigenen Studie zur Schweregradbestimmung eingesetzt.

\subsubsection{Severity of Alcohol Dependence Questionnaire (SADQ)}

Mit dem SADQ (Stockwell et al. 1979) wurde zum ersten Mal versucht, den Schweregrad des von Edwards und Gross beschriebenen Abhängigkeitssyndroms zu erfassen. Die Skala erfragt die letzte Trinkepisode, erfasst aber nicht alle Symptome des Abhängigkeitssyndroms nach Edwards und Gross. Der Fragebogen besteht aus 20 Items und wird in fünf Subskalen eingeteilt: körperliche Entzugserscheinungen, affektive (psych.) Entzugserscheinungen, Craving und entzugserleichterndes Trinken, typische Tageskonsummenge, Wiederherstellung des Syndroms nach einer 
Abstinenzperiode. Sie konzentriert sich relativ stark auf körperliche Symptomatik der Alkoholabhängigkeit.

\subsubsection{Göttinger Abhängigkeitsskala (GABS)}

Die Göttinger Abhängigkeitsskala wurde als deutsche Version des SADQ von Jacobi et al. 1987 entwickelt. Als Selbstbeurteilungsfragebogen mit 20 Items dient die Skala der Erfassung des Schweregrades der Alkoholabhängigkeit. Sie erfasst folgende Abhängigkeitsmerkmale; Einengung des Trinkverhaltens, übergroße Bedeutung des Alkohols, erhöhte Alkoholtoleranz, wiederholt auftretende Entzugssymptome, Trinken zur Beseitigung der Entzugssymptomatik, unwiderstehliches Verlangen zu trinken sowie das erneute Auftreten des Syndroms nach Abstinenzphasen.

Insgesamt lässt sich feststellen, dass alle vorgestellten Testverfahren mehrdimensionale Instrumente sind, die auf unterschiedlichen Stufen die Alkoholabhängigkeit und ihren Schweregrad beurteilen. Der EuropASI Fragebogen und die SESA basieren jeweils auf sehr umfangreichen Untersuchungen und weisen hohe Testgütekriterien auf. Deshalb kamen diese bei der vorliegenden Studie zum Einsatz.

In manchen Studien wurde zur Bestimmung des Schweregrads ein ganz anderes Kriterium benutzt, nämlich die Trinkmenge. Allerdings ist die Grenze zwischen einem problematischen oder gefährlichen Alkoholkonsum schwer zu bestimmen. In einer Übersichtsarbeit teilen Bühringer et al. 2002 die Konsumenten in vier Risikobereiche auf. Zu einer Gruppe mit niedrigerem Risiko gehören Männer mit einem täglichen Konsum von $30 \mathrm{~g}$ reinem Alkohol (Frauen $20 \mathrm{~g}$ ). Zu der Kategorie des problematischen Alkoholkonsums gehören Männer, die täglich 30 bis $60 \mathrm{~g}$ Alkohol trinken (Frauen 20 bis $40 \mathrm{~g}$ ). In der Kategorie des schädlichen Konsums sind diejenigen, die täglich 60 bis $120 \mathrm{~g}$ Alkohol trinken (Frauen 40 bis $80 \mathrm{~g}$ ). In der 
Kategorie des Hochrisikokonsums befinden sich schließlich diejenigen, die mehr als 120 g. Alkohol täglich konsumieren (Frauen 80 g). Der Schweregrad wird leider auf Grundlage unterschiedlicher Basisangaben der Trinkmenge bei mehreren Studien verwendet (Adinoff et al., 2017), je nachdem, welches Forschungsziel die jeweilige Studie verfolgt. Wenn es beispielsweise um Effekte des langjährigen hochriskanten Alkoholkonsums geht, wurden bei mehreren Studien die Trinkmengen als Schweregradkriterium benutzt. So konnten beispielsweise in einer Studie mit 46 chronisch stark trinkenden Alkoholikern (Heavy Drinker, 80-100 „drinks“/Monat) und 52 moderat trinkenden Alkoholikern (Light Drinker, 35-45 „drinks“/Monat) signifikant geringere N-Acetyl-Aspartat-(NAA)-Konzentrationen in der weißen Substanz des Frontalhirns bei stark trinkenden im Vergleich zu moderat trinkenden Alkoholikern in einer MR-spektroskopischen Untersuchung gefunden werden (Meyerhoff et al., 2004). Auch eine Korrelation zwischen Schweregrad des Trinkens und den Konzentrationen der Metaboliten Kreatin und Cholin konnte bei schwerkranken Alkoholikern in dieser Studie festgestellt werden. Eine ähnliche neuere Studie konnte einen signifikanten negativen Zusammenhang zwischen der Schwere der Alkoholabhängigkeit und der Dichte der grauen Substanz finden (Bullock et al., 2017). Eine weitere ähnliche Untersuchung wurde von Andreas Bartsch und Mitarbeitern zur Frage der frühen cerebralen Regeneration während der Abstinenzphase bei Alkoholabhängigen durchgeführt (Bartsch et al., 2007).

Eine umfassende Beurteilung des Schweregrads auf der Basis der Trinkmenge, auch unter Berücksichtigung von Trinkmustern, Anzahl von Entzugsbehandlungen oder Suchtdruck, ist aus klinischer Sicht völlig unzureichend. Denn Alkoholabhängige zeigen nicht nur eine schwere Krankheitssymptomatik, wenn sie hohe Dosen an Alkohol trinken. Auch weitere Symptome müssen berücksichtigt werden. So zeigen Patienten mit einem höheren Schweregrad der Alkoholabhängigkeit stärkeres 
Verlangen nach Alkohol (Yoon et al., 2006) als auch längere Trinkdauer (Tolic \& Soyka, 2018).

Es können schließlich auch standardisierte Biomarker bei der Beurteilung der Schwere alkoholbezogener Störungen hilfreich sein. (Niemelä, 2016). Aber auch sie können lediglich Hinweise auf den Krankheitsschweregrad liefern und sind als alleiniges Kriterium ebenfalls unzureichend. Ein möglicher Weg könnte in einer Kombination aus psychologischen Testverfahren (z. B. SESA, EuropASI) sowie konventionellen (z. B. Gamma- GT, GOT, GPT, MCV) und neueren Biomarkern (z. B. CDT) bestehen, um konsistentere Ergebnisse zu bekommen. Diese können dann dem jeweiligen Schweregrad der Abhängigkeit zugeordnet werden. Auch Stress spielt bei dem Schweregrad der Alkoholabhängigkeit eine wichtige Rolle. Neuere Studien weisen darauf hin, dass dysfunktionale Veränderungen der HHNA mit dem Schweregrad der Abhängigkeit assoziiert sind (Blaine \& Sinha, 2017). Je höher die chronische Stressbelastung, desto höher wird meist der Schweregrad der Alkoholabhängigkeit und die Trinkintensität (Trinkmenge) bei einem Rückfall (Adinoff et al., 2017). Umgekehrt: je höher der Schweregrad der Alkoholabhängigkeit, desto höher die chronische Stressbelastung und das Flucht- und Vermeidungsverhalten (Tolic \& Soyka, 2018). Die Studie von Boschloo et al. (2012) zeigt, nur als Beispiel hier erwähnt, die Wichtigkeit des Schweregrads als Prädiktorvariable sowohl für die Aufrechterhaltung als auch für das Rückfallrisiko bei einer Alkoholabhängigkeit. Deshalb ist seine Bestimmung für die Durchführung individualisierter Therapien relevant. Es stellt sich aber gleichzeitig die Frage, wie man den Schweregrad ökonomisch am besten erfassen und sinnvoll in einen diagnostischen und therapeutischen Prozess integrieren kann. In der Zukunft wird sich zeigen, welche weitere Methoden, vielleicht epigenetische, genauere Ergebnisse liefern und 
somit die Beurteilung der Abhängigkeitsschwere erleichtern können (Brückmann et al., 2016). Der Bedarf nach zuverlässigen Instrumenten dürfte sich nicht vermindern angesichts der Tatsache, dass das DSM- 5 seine kategoriale Einteilung in Missbrauch und Abhängigkeit aufgehoben hat und die Kriterien auf der Basis des Schweregrads der Substanzgebrauchsstörung eingeführt hat.

\subsection{Abstinenzdauer}

Alkoholabstinenz als freiwilliger oder unfreiwilliger Verzicht auf Alkohol ist Aufgrund der unterschiedlichen Zeiträume und unterschiedlicher Genauigkeit hinsichtlich der Trinkmenge (absolute Abstinenz vs. ein Glas zum Geburtstag erlaubt) nicht leicht definierbar. In der einschlägigen Literatur finden sich bei den Studien unterschiedliche Angaben hinsichtlich der Abstinenzdauer bei Untersuchungsteilnehmern. Diese reichte von zwei bis sechs Wochen (Junghanns et al., 2003) bis zu 3,5 bis 5 Jahren (Munro et al., 2005). In einer Studie betrug die Abstinenzdauer bei einer Probandengruppe sogar nur 24 Stunden (Starcke et al., 2013). Eine allgemeinverbindliche Regelung existiert nicht. Ohne Komplikationen bleibt ein Alkoholentzugssyndrom nur "wenige Tage“ bestehen und bildet sich innerhalb einer Woche zurück (Soyka \& Küfner, 2008, s. 171). Unter Berücksichtigung weiterer Einschlusskriterien wurden demnach solche Patienten mit 7-tägiger Abstinenz als geeignet eingestuft und in die vorliegende Studie aufgenommen. Mit einer Normalisierung der Hypothalamus-Hypophysen-Nebennierenrinden-Achse (HHNA) rechnet man bei Alkoholabhängigen nach etwa 8-wöchiger (Coiro et al., 2007), spätestens aber nach 12-wöchiger Abstinenz (Ehrenreich et al., 1997). Es besteht eine hohe Wahrscheinlichkeit, dass die Normalisierung der HHNA-Aktivität mit der Abstinenzdauer im Zusammenhang steht (Stephens et al., 2014). Studienergebnisse 
weisen darauf hin, dass die Abstinenzdauer auch mit der Höhe der Cortisolkonzentration in positivem Zusammenhang steht (Adinoff et al., 2005; Kiefer et al., 2002; Starcke et al., 2013). Bei der Normalisierung der HHNA-Funktion bei Alkoholabhängigen spielen verschiedene Faktoren eine wichtige Rolle, wie Schweregrad der Alkoholabhängigkeit, evtl. Kindheitstraumata, Schwere der Rückfallsymptomatik, Genetik und die individuellen Reaktionsmuster auf Stress (Schäfer et al., 2010; Bernardy et al., 1996). Der genauere Ablauf, wie sich die HHNA-Funktion wieder normalisiert bzw. unter welchen Bedingungen, ist immer noch unklar. In Anbetracht dieser Befunde und der Einteilung in der Deutschland üblichen Dauer stationärer Entwöhnungsbehandlungen für Alkoholabhängige als medizinische Rehabilitation Abhängigkeitskranker (ca. 6 bis 15 Wochen) wurde so auch die zweite Gruppe der Patienten mit einer 70-tägigen Abstinenz in die eigene Studie aufgenommen (Tolic \& Soyka, 2018). In der frühen Abstinenzphase, insbesondere vor Beendigung einer stationären Entzugs- (ca. 7 bis 14 Tage) oder Entwöhnungsbehandlung (ca. 42 bis 105 Tage), werden die Patienten mit zahlreichen, manchmal auch recht stressigen Herausforderungen des alltäglichen Lebens konfrontiert. In der frühen Abstinenzphase konnte verminderte Stressreagibilität bei Alkoholabhängigen (Junghanns et al. 2003; Tolic \& Soyka, 2018) und ein erhöhtes Rückfallrisiko festgestellt werden (Junghanns et al. 2003). In dieser Phase ereignen sich offenbar auch die meisten Alkoholrückfälle (Sinha et al. 2007; Blaine et al., 2016).

\section{Therapie}

Alkoholbezogene Störungen werden in ihrer Entstehung auf der Basis eines bio-psycho-sozialen Bedingungsmodells verstanden. Dementsprechend ist eine sorgfältige Analyse und Beeinflussung in allen betroffenen Bereichen sinnvoll und 
notwendig. Die Behandlung besteht meist aus einer Kombination allgemeinmedizinischer, suchtmedizinischer, psychotherapeutischer, psychopharmakotherapeutischer, sozialtherapeutischer und zahlreicher anderer Maßnahmen (z. B. Sporttherapie, Ergotherapie, Musiktherapie). Je nach Art und Schwere der alkoholbezogenen Störung sowie meist noch vorhandener und sehr relevanter Patientenmerkmale (Kompetenzen, Ressourcen) wird entsprechend der Therapieumfang bestimmt (Geyer et al., 2006). Die therapeutischen Versorgungsmöglichkeiten von Patienten mit alkoholbezogenen Störungen in Deutschland sind sehr komplex. Vereinfacht können diese in einem Phasenmodell mit vier Phasen der Behandlung dargestellt werden. In der Kontakt- und Motivationsphase steht der Aufbau und Festigung der Behandlungsmotivation im Vordergrund. Hinzu kommen Beratung, Anamneseerhebung sowie medizinische- und psychologische Diagnostik mit dem Ziel, einen Behandlungsplan zu erstellen. Die Phase der Akutbehandlung (Entzug) ist durch die Behandlung von Alkoholintoxikation, der Entzugssymptome und Vorbeugung von Komplikationen gekennzeichnet. Die Entwöhnungsphase hat zum Ziel, durch umfassende Maßnahmen der Rückfallvermeidung die Abstinenz langfristig zu stabilisieren. Die Entwöhnungsbehandlungen werden mittels integrierter Therapieelemente in ambulanten, teilstationären und stationären Settings durchgeführt. Schließlich sollten in der Nachsorgephase die gelernten Inhalte in den Alltag transferiert werden und eine berufliche Wiedereingliederung erfolgen.

Pharmakotherapie

Eine medikamentöse Therapie findet sowohl in der Akutbehandlung als auch in der Langzeittherapie alkoholbezogener Störungen ihre Anwendung. Insbesondere in der Akutbehandlung sind Medikamente häufig unverzichtbar und werden zur Linderung 
der Entzugssymptome als auch zur Vorbeugung von Komplikationen eingesetzt (AWMF: S3-Leitlinie, 2016). Zum Einsatz kommen meist Clomethiazol, Benzodiazepine, Clonidin, Antiepileptika und Antipsychotika (AWMF: S-3 Leitlinie, 2016). Für die Indikation einer Langzeittherapie der Alkoholabhängigkeit sind in Deutschland drei Medikamente zugelassen, nämlich Acamprosat, Naltrexon und Nalmefen. Die antagonistische Wirksamkeit von Acamprosat an Glutamat-Rezeptoren ist gut erforscht. Obwohl seine Wirksamkeit statistisch als bewiesen gilt, wird sie aber als gering bezeichnet (Jonas, et al., 2014). In der Behandlungsgruppe mit Acamprosat blieben $9 \%$ mehr Patienten abstinent als in der Placebo-Gruppe (Jonas et al., 2014). Eine noch geringere Wirksamkeit wird dem Opiatantagonisten Naltrexon zugeschrieben. Es gibt Hinweise, dass seine Wirksamkeit vorwiegend auf Reduktion von exzessivem Trinken basieren könnte (Pettinati et al., 2006). Die sorgfältig durchgeführte Metaanalyse von Jonas und Mitarbeitern besagt, dass in der Naltrexon-Gruppe nur $5 \%$ mehr Patienten abstinent blieben als in der PlaceboGruppe (Jonas et al., 2014). Bei Nalmefen handelt es sich ebenfalls um einen Opiatantagonisten, der aber nicht zur Aufrechterhaltung der Abstinenz, sondern zur Reduktion der Trinkmenge zugelassen wurde. Die Studienlage zu Nalmefen ist wesentlich dünner als zu Acamprosat und Naltrexon. Seine Wirksamkeit wird als sehr gering eingeschätzt (Mann et al., 2013). Die Zulassung für Disulfiram (Antabus®) ist 2013 erloschen.

Psychotherapeutische Methoden

Eine Psychotherapie beabsichtigt, das Erleben und Verhalten einer Person zu verändern mit dem Ziel, dysfunktionale Gedanken, Gefühle, Erwartungen und Reaktionen abzubauen und defizitäre Bewältigungskompetenzen aufzubauen (Chambless \& Ollendick, 2001). Somit kann der betroffenen Person zu einer 
Verbesserung der psychischen und körperlichen Gesundheit sowie zur besseren Integration in der Gesellschaft geholfen werden. Die wichtigste Voraussetzung für eine erfolgreiche Psychotherapie ist eine vertrauensvolle und tragfähige therapeutische Beziehung (Horvath \& Luborsky, 1993; Horvath et al., 2011). Oft beeinflussen die spezifischen Fähigkeiten des Therapeuten das Behandlungsergebnis in höherem Ausmaß als die verwendete Methode (Andreasson \& Öjehagen, 2003). Das Ziel besteht darin, dass der oft von außen (Familie, Arbeitgeber) erzeugte Veränderungsdruck in eine persönliche Änderungsmotivation umgewandelt wird. Nach dem Erreichen einer Abstinenz in der Postakutphase besteht das größte Ziel in der Rückfallprävention mittels Verbesserung des Selbstmanagements im Umgang mit Alkohol. Dies geschieht vor allem durch gezielte Stabilisierung der Abstinenzmotivation als auch durch Aufbau erfolgreicher Steuerungsfähigkeit hinsichtlich Alkoholgedanken und Alkoholverlangen. Auch motivierende Gespräche als Interaktionsstil sollten eingesetzt werden (Miller \& Rollnick, 1999). Die Ausarbeitung der Risikosituationen gehört ebenso dazu wie der Aufbau alternativer Verhaltensweisen. Nicht selten haben Alkoholkranke die Überzeugung, dass ein abstinentes Leben gar nicht möglich ist, weil sie sich nicht fähig fühlen, ohne Alkohol zu leben. Diese Überzeugung ist häufig die Grundlage für die geringe Änderungsmotivation. Deshalb wird der Aufbau der Änderungsmotivation als wichtiger Bestandteil der Psychotherapie angesehen (Veltrup, 2002). Die Effektstärke von Psychoedukation als alleinige Intervention ist gering und sollte nur als integraler Bestandteil einer komplexen Behandlung angeboten werden (Miller \& Wilbourne, 2002). Im Gegensatz dazu finden Methoden und Techniken der kognitiven Verhaltenstherapie die häufigste Anwendung. Sie sind erfolgreich und werden deshalb empfohlen (Morgenstern \& Longabaugh, 2000; Durham et al., 2005). Die Verhaltenstherapie kann sowohl für die Behandlung von alkoholbezogenen Störungen 
als auch für die Therapie komorbider psychischer Störungen und zur Rückfallprophylaxe angewandt werden (Rist, 2002; Morgenstern \& Longabaugh, 2000). Ihre Anwendung erzielt gute Ergebnisse, auch wenn der Schweregrad der Alkoholabhängigkeit hoch war (Roos et al., 2017). Der Einsatz individualisierter Therapien wird grundsätzlich als noch erfolgreicher beschrieben (Litt et al., 2009). Ebenso wirksam ist das soziale Kompetenztraining, das sehr gut mit anderen Therapiemodulen kombinierbar ist und sollte aus diesem Grund im Rahmen einer integrativen Therapie nicht fehlen (Miller \& Wilbourne, 2002). In der Phase der Postakutbehandlung können auch weitere Therapien erfolgreich angewendet werden, wie beispielsweise die psychodynamischen Therapien. Sie sollen insbesondere bei weniger stark Abhängigen gute Ergebnisse erbringen (Kadden et al., 2001). Zur Verbesserung hoher kognitiver Funktionen (Aufmerksamkeit, Planung, Flexibilität) können Übungen der Ergo- und Arbeitstherapie einen wichtigen Beitrag leisten und den Patienten für die berufliche Wiedereingliederung gut vorbereiten (Deister, 2005). Alkoholkranke ohne Arbeit oder gesicherte Wohnverhältnisse zeigen schlechtere Therapieergebnisse (Henkel, 2011). Sozialtherapeutische Interventionen haben deshalb zum Ziel, die Befähigung des Patienten, seine sozialen Verhältnisse eigenverantwortlich zu regeln. In einem ganzheitlichen Therapieprogramm sollten Körpertherapien (Entspannungsverfahren, Sport) ebenso wie die Paar- und Familientherapie nicht fehlen. Sie sollten aber nicht als alleinige Therapiemethode angewandt werden (Miller \& Wilbourne, 2002). Die sich nach einer Entwöhnungsbehandlung anschließende ambulante Nachsorge ergänzt die stationäre Therapie und kann in einer stationären oder ambulanten Form durchgeführt werden. Sie dient der Aufrechterhaltung der Abstinenz, der Stärkung der Selbstwirksamkeit als auch dem Transfer der erlernten Verhaltensweisen ins allägliche Leben. Sie kann beispielsweise in Form einer professionellen Behandlung und/oder in Form 
regelmäßige Selbsthilfegruppenbesuche als auch betreutem Wohnen erfolgen. Inzwischen existieren verschiedene Modelle und Abfolgen stationär-ambulanter und ambulant-stationärer Behandlungen. Für die gemeindenahe Behandlung und Betreuung von Betroffenen sorgen außerdem zahlreiche niedergelassene Ärzte und Psychotherapeuten. Wenn eine Abstinenz vorliegt, das Therapiesetting als geeignet erscheint und die suchttherapeutischen Kompetenzen vorhanden sind, dann können die niedergelassenen Ärzte und Psychotherapeuten langfristig bedeutsame Hilfen für die Betroffenen von alkoholbezogenen Störungen anbieten (Hapke et al., 2003). Schließlich kann die Teilnahme an einer Selbsthilfegruppe für die Betroffenen hilfreich sein.

\section{Stress}

Das Wort „Stress“ geht auf das lateinische „stringo“ und „stringere“ zurück, was so viel wie „straff anziehen“ und „zusammenziehen“ bedeutet. Der Begriff an sich existierte auch zwischen 1900 und 1950, aber in diesem Zeitraum wurde kaum zum Thema Stress publiziert. Erst in der zweiten Hälfte des 20. Jahrhunderts, etwa ab den 60er-Jahren, nahmen die Stressstudien deutlich an Zahl zu. In den 70er-Jahren kam es zu einer ganzen Flut an Veröffentlichungen zum Thema Stress. So wurde Stress innerhalb von wenigen Jahrzehnten zu einer festen Größe der medizinischen und psychologischen Forschung. Es handelt sich also um ein verhältnismäßig junges Konzept, was sehr eng mit unserer modernen Zeit und unserer Lebensweise verknüpft ist. In der Physik und den Ingenieurwissenschaften wird der Begriff benutzt, um Beanspruchungen von Materialien zu beschreiben. In der Alltagssprache wird der Begriff "Stress" meist mit bedrohlichen und allgemein unangenehmen Gefühlszuständen assoziiert. Er kann als Oberbegriff sowohl für den 
stressauslösenden Reiz (Stressor) und die darauffolgende Stressreaktion als auchfür die psychobiologischen Mediatorvariablen verwendet werden, wobei im alltäglichen Sprachgebrauch eine solche Unterscheidung eher selten vorgenommen wird. In der wissenschaftlichen Literatur findet man sogar innerhalb eines Fachbereichs recht unterschiedliche Stressdefinitionen. Aus diesem Grund gibt es Vorschläge, den Terminus Stress nur auf diejenigen Ereignisse zu begrenzen, die als unkontrollierbar oder unvorhersehbar wahrgenommen werden und eine physiologische und behaviorale Stressreaktion im Organismus auslösen (Koolhaas et al., 2011). Die Autoren empfehlen, bei rein physischen Belastungen nicht vom Stress zu sprechen. Insgesamt besteht in der Fachwelt eine Einigkeit darüber, dass der Stress einen Versuch des Organismus darstellt, sich den verschiedenen Belastungen anzupassen. Gerade durch die Anpassung an gesellschaftliche Veränderungen und Veränderungen in der Arbeitswelt steigt die psychische Belastung beim Menschen in den letzten Jahrzehnten stetig an (Landsbergis et al., 2014).

\subsection{Stressmodelle}

Bereits 1897 konnte der amerikanische Physiologe Walter Bradford Cannon bei den Tierversuchen beobachten, dass bei Furcht die peristaltischen Wellen des Magens plötzlich aufhörten. Er interessierte sich für die Physiologie der Emotionen und ging in seinen Arbeiten davon aus, dass menschliche Handlungen ihren Ursprung in Emotionen haben (Brown \& Fee, 2002). So prägte er den Begriff der „Fight or Flight-Reaktion“, nachdem er in Tierexperimenten beobachtet hatte, dass unterschiedliche Reize (z. B. Kälte oder Hitze) bei Versuchstieren zu einer körperlichen Anpassungsreaktion führten, die sich in Form von Erhöhung von Blutdruck und Herzfrequenz zeigten. Er ging davon aus, dass der Organismus physiologisch nach einem über verschiedene Regelkreise steuernden Gleichgewicht 
(Homöostase) strebt. Unter Homöostase verstand Cannon die Grundannahme, dass es für alle physiologischen Systeme (z. B. Körpertemperatur, Blutdruck, Herzfrequenz, usw.) einen Sollwert gibt, der durch entsprechende Regulationsmechanismen aufrechterhalten werden soll (Brown \& Fee, 2002). Cannon sprach von Störreizen, die die Homöostase des Organismus verändern und dadurch eine physiologische Alarmreaktion zur Wiederherstellung des Gleichgewichts auslösen. Die physiologischen und neuromuskulären Reaktionen auf die Störreize wurden von Cannon nicht weiter unterteilt. Der kanadische Endokrinologe Hans Selye ging in seinen Studien zur Reaktion auf einen andauernden (chronischen) Stressor einen wichtigen Schritt weiter. Er formulierte ein unter dem Namen "Allgemeines Adaptationssyndrom" bekannt gewordenes Stresskonzept (Selye, 1950). In diesem unterteilt er die Reaktion auf einen Stressor in drei Phasen, nämlich Alarmphase (Alarmreaktion), Widerstandsphase und Erschöpfungsphase. Die Einwirkung des Stressors bewirkt nach Selye $(1950,1998)$ zunächst eine Alarmreaktion, die sich beispielsweise in Form einer Hypertrophie verschiedenster Organe oder Absenkung der Körpertemperatur innerhalb von 6-48 Stunden manifestiert. Bleibt die Einwirkung des Stressors weiter aufrecht, geht die Alarmreaktion in die Widerstandsphase über. Diese zeichnet sich durch erhöhte Toleranz gegenüber dem Stressor aus, die aber als Anpassung zeitlich klar begrenzt ist. Beim Fortbestehen des Stressreizes bricht die Anpassung zusammen und der Organismus erleidet in der darauffolgenden Erschöpfungsphase anhaltende, teilweise irreversible Schäden. Aber der Stress ist komplexer als nur die Störung der Homöostase (Miller \& O'Callaghan, 2002). Diese Befunde basierten auf tierexperimentellen Untersuchungen und sind daher nur bedingt auf den menschlichen Organismus übertragbar. Die Kritik wurde natürlich noch deutlicher, als man entdeckte, dass auch eine Antizipation der Bedrohung (z. B. bei Prüfungen) ausreicht, eine 
physiologische Stressantwort auszulösen. Deshalb wurde das Konzept der Homöostase durch Sterling und Eyer (1988) erweitert und in das Konzept der „Allostase“ umbenannt. Dieses beschreibt im Wesentlichen, dass es für die einzelnen physiologischen Parameter keinen bestimmten Sollwert gibt, sondern dass sich die physiologischen Prozesse dem aktuellen Zustand des Organismus anpassen (z. B. Bewegung, Schlaf). Das Konzept wurde später von der Forschergruppe um Bruce McEwen weiterentwickelt (McEwen, 1998, McEwen, 2003, McEwen, 2004). Einen anderen Ansatz verfolgte die Forschergruppe um Holmes und Rahe, 1967. Diese zwei Autoren postulierten, dass nicht nur akut negative Ereignisse Stress auslösen, sondern auch einschneidende Lebensereignisse (Life Events) wie beispielsweise Heirat oder die Geburt des Kindes. Die individuelle Wahrnehmung und die Bewertung des Stressreizes spielt bei der Entstehung von Stressreaktion eine wichtige Rolle. Schließlich gewannen die psychologischen Variablen in der Stressforschung immer mehr an Bedeutung. So konnte Richard Lazarus in seinem Transaktionsmodell weitgehend die Komplexität des Stressphänomens abbilden und stellte die Person sowohl als reaktive (auf Umweltreize) als auch handelnde (auf die Umwelt einflussnehmende) Instanz dar (Lazarus \& Folkman, 1984). Der Mensch ist nach Lazarus und Folkman (1984) seinen eigenen oder Umweltstressoren keineswegs passiv ausgesetzt, sondern setzt sich mit diesen aktiv auseinander. Ob ein Reiz überhaupt zu einem Stressreiz wird, hängt von der Bewertung ab. Die kognitive und die emotionale Antwort auf einen Stimulus hat auch einen entscheidenden Einfluss darauf, ob überhaupt und wie intensiv eine neuroendokrine Stressreaktion ausgelöst wird. Wenn die Person die Situation als subjektiv bedeutsam einschätzt, weil sie beispielsweise wichtige Lebensbereiche, Ziele und Bedürfnisse bedroht und zu der Einschätzung kommt, dass mit den vorhandenen Ressourcen die Situation nicht bewältigt werden kann, dann entsteht Stress. Erst die 
subjektiv wahrgenommene Diskrepanz zwischen den Stimuluseigenschaften einerseits und den eigenen Fähigkeiten und Ressourcen andererseits führt nach dem Transaktionsmodell zu Stress. Die Entwicklung des Transaktionsmodells regte die Stressforschung in beträchtlichem Ausmaß an. Der Forschergruppe um Dickerson und Kemeny ist es beispielsweise gelungen, einen noch differenzierteren Ansatz zu entwickeln. Sie postulierten, dass der Mensch dann Stress erlebt, wenn er eine Anforderung als unkontrollierbar einschätzt und/oder von anderen Personen während einer Aufgabe negativ bewertet werden kann (Dickerson \& Kemeny, 2004). Wenn beide Aspekte in einer Anforderung vorhanden sind, könnten diese die stärkste und längste Stressreaktion im Körper hervorrufen. Diese Erkenntnis ist sowohl für die therapeutischen Anwendungen in der Praxis (Expositionsbehandlung) als auch für die experimentelle Stressinduktion in der Laborforschung von Bedeutung. Diese Methode führt zu einer signifikanten Erhöhung des Cortisolwertes (Dickerson \& Kemeny, 2004; Kudielka \& Kirschbaum, 2005; Kudielka et al., 2009).

\subsection{Stressreaktion: Darstellung des Ablaufs und der beteiligten biologischen Stressachsen}

Wie im vorangegangenen Abschnitt dargestellt, versucht der menschliche Organismus mittels Stressreaktionen sich wechselnden Bedingungen in seiner Umwelt anzupassen. Für diese lebensnotwendigen Anpassungen sorgen zwei bedeutende Stresssysteme; das sympatho-adrenomedulläre System (SAM) und die Hypothalamus-Hypophysen-Nebennierenrinden-Achse (HHNA). Ihre Aktivierung kann durch verschiedenste psychosoziale und physische Stressoren erfolgen. 


\subsubsection{Das sympatho-adrenomedulläre System (SAM)}

Folgt man den oben beschriebenen Forschungsarbeiten, strebt der Organismus in der Auseinandersetzung mit den Reizen aus der Innen- und Außenwelt nach einer Homöostase. Eine Stressreaktion auf diese Reize erfolgt sowohl auf zentralnervöser als auch peripherphysiologischer Ebene. Je nach Beschaffenheit des Stressors werden bei der Stressreaktion unterschiedliche Organe und Organsysteme aktiviert. So auch das vegetative Nervensystem mit seinen beiden Nervensträngen Sympathikus und Parasympathikus. Der Sympathikus übernimmt die leistungsfördernde und der Parasympathikus eine ruhefördernde und erholende Funktion. Diese Funktionen erfolgen über unterschiedliche Nervenfasern mit unterschiedlichen Überträgersubstanzen. Die präganglionären Fasern des Sympathikus, d. h. diejenigen, die vom ZNS zum Ganglion führen, sind cholinerg und die postganglionären, die zum Effektororgan führen, vorwiegend adrenerg. Die über sensorische Organe aufgenommenen Informationen werden zunächst auf der Ebene des limbischen Systems, insbesondere im Thalamus, verarbeitet, wo ein erstes rudimentäres Bild der Situation entsteht. Vom Thalamus aus gelangen die Informationen zum Neokortex (Hirnrinde), wo sie in einem komplexeren Vorgang verarbeitet werden. Wenn der Reiz als potenziell bedrohlich eingeschätzt wird, erfolgt eine Involvierung der Amygdala, wo die neuronale Erregung eine affektive Färbung erhält. Von der Amygdala aus bestehen direkte neuronale Verbindungen zum Locus coeruleus (LC), wo es zur vermehrten Ausschüttung von Noradrenalin und zu einer Stimulierung des sympatho-adrenergen Systems kommt (Diorio \& Meaney, 2007). Eine Stressreaktion entsteht. Die Amygdala ist mit zahlreichen Hirnarealen, auch über die Corticotrope-Releasing-Hormon-Neurone (CRH-Neurone), verbunden (Koob, 2008) und ist an der Beeinflussung der SAM ebenso beteiligt wie der mediale 
präfrontale Cortex (mPFC). Im weiteren Verlauf der Stressreaktion wird aus den Vesikeln sympathischer Nervenendigungen Noradrenalin freigesetzt, womit periphere Effektororgane aktiviert werden und schließlich auch das Nebennierenmark stimuliert wird, Katecholamine, Adrenalin (80\%) und Noradrenalin (20\%) in die venöse Blutbahn freizusetzen (Birbaumer \& Schmidt, 2010). Außerdem wird über afferente Bahnen und vorwiegend über den Nervus Vagus, der Nucleus Tractus Solitarius (NTS) im Hirnstamm aktiviert. Dieser befindet sich in einem Netzwerk wichtiger noradrenerger, adrenerger und peptiderger Verbindungen, insbesondere zum LC und paraventrikulären Kern des Hypothalamus (Übersicht bei Herman et al., 2016; Ulrich-Lai \& Herman, 2009). Bei den noradrenergen Prozessen im Cortex wird dem NTS und dem Hypothalamus eine zentrale Rolle zugeschrieben (Herman et al., 2016; Ulrich-Lai \& Herman, 2009). In Stresssituationen kann der paraventrikuläre Kern des Hypothalamus mit seiner Verbindung über NTS zum LC Einfluss auf den Sympathikus ausüben und die Freisetzung von Noradrenalin im Cortex in Abhängigkeit von Schweregrad und Dauer des Stressors regulieren. NTS und LC sind aber nicht nur bei der Regulierung des SAM, sondern auch der HHNA beteiligt (Ulrich-Lai \& Herman, 2009). Im Rahmen der Stressreaktion werden auch weitere Neurotransmitter im Cortex ausgeschüttet wie Corticotropin-Releasing-Hormon (CRH), Serotonin und Dopamin. So übernimmt das CRH mit dem LC/-Noradrenalin-Sympathikus-System die wichtigste Rolle bei der Regulierung des SAM. Über interaktive und Synergieeffekte der beiden Stressachsen siehe weiter unten.

Die Aktivität des sympatho-adrenomedullären Systems (SAM) gilt bei der Stressreaktion als eine Art "erste Reaktionsstufe" im Organismus und ist gleichermaßen an der Stressreaktion beteiligt wie die HHNA (Kirschbaum et al., 1993). Diese Alarmfunktion dauert meist kurz, nur wenige Minuten und bereitet den Organismus auf eine effiziente „Kampf oder Flucht“-Reaktion vor (Habib et al., 2001; 
Herman et al., 2016; Ulrich-Lai \& Herman, 2009). Unter anderem kommt es zur Erhöhung der Atemfrequenz, Bronchodilatation, Vasokonstriktion in den Hautpartien, aber Vasodilatation in den Arterien der Effektororgane (für die notwendige Arbeit der großen Muskeln), Energiemobilisierung, Erhöhung des Herzschlags, aber auch zur Unterdrückung der Verdauung und der Sexualfunktionen (Herman et al., 2016; Kaltsas \& Chrousos, 2007, Habib et al., 2001).

\subsubsection{Hypothalamus-Hypophysen-Nebennierenrinden-Achse (HHNA)}

Ihre Funktionsweise wurde erstmalig 1936 von Hans Selye beschrieben, als er bei seinen Experimenten die Stressreaktionen an Ratten untersuchte. Bei der Hypothalamus-Hypophysen-Nebennierenrinden-Achse (HHNA) handelt es sich um eine teilweise selbstregulierende endokrine Einheit, die bei essentiellen physiologischen Funktionen und grundsätzlich bei der Aufrechterhaltung der homöostatischen Prozesse eine wichtige Rolle spielt. Wird der Stimulus im Frontalhirn für den betroffenen Organismus als bedrohlich bewertet, dann erfolgen die Stressantworten mit der Aktivierung der SAM und meist auch der HHNA. Der Beginn der HHNA-Aktivierung erfolgt im paraventrikulären Kern des Hypothalamus (PVN), wo in den neuroendokrinen Zellen das Corticotropin-Releasing-Hormon ( $\mathrm{CRH}$ ) und Arginin-Vasopressin (AVP) gebildet und sezerniert werden. Dem $\mathrm{CRH}$ wird sowohl die größte physiologische Aktivierungsfunktion für die HHNA als auch in der Folge die wichtigste regulatorische Funktion der Stressreaktion beim Menschen zugeschrieben (Dedic et al., 2018). In der parvozellulären Region des PVN wird die CRH-Aktivität durch verschiedene Neurotransmittersysteme moduliert, wie GABA, Opioide, Glutamat und Serotonin (Stephens et al., 2014). Über das portale Blutgefäßsystem gelangt das $\mathrm{CRH}$ zum Hypophysenvorderlappen und bindet in der kortikotropen Zellregion an den Cortiocotrope-Relasing-Hormon-Rezeptor 1 (CRHR1). Dort stimuliert CRH die 
Freisetzung des adrenocorticotropen Hormons $(\mathrm{ACTH})$ in den Blutkreislauf (Hermann et al., 2016). ACTH ist als Derivat des Vorläuferhormons Proopiomelanocortin (POMC) ein aktives Peptid, das in der Nebennierenrinde die Synthese und Freisetzung von Cortisol als dominantestem Glukokortikoidhormon beim Menschen stimuliert (Kaltsas \& Chrousos, 2007; Kirschbaum \& Hellhammer, 1999). Das freigesetzte Cortisol ermöglicht eine Anpassung an die vorliegenden Stressbedingungen. Die Anpassung erfolgt in Abhängigkeit von der Intensität der Stressreizwirkung und in entsprechender Länge. Etwa eine Stunde hält sich die Cortisolkonzentration im Blut aufrecht. Schätzungsweise wird die basale Konzentration des Cortisols 60-90 Minuten nach Wegfall des Stressors wieder erreicht (De Kloet et al., 2005). Die Höhe der CortisolKonzentration im systemischen Blutkreislauf sorgt nicht nur für die lebensnotwendigen Anpassungen, sondern erzeugt gleichzeitig auch die Hemmung der HHNA-Aktivität. In diesem Sinne reguliert sich das System im Rahmen einer negativen Rückkopplung durch die Bindung an spezifische Rezeptoren im Gehirn selbst (de Kloet et al., 2018; Joëls, 2018). Diese negative Feedbackaktivität beginnt bei hoher CortisolKonzentration in der stressreaktiven Phase mit der Bindung des Cortisols an den niedrigaffinen Glukokortikoidrezeptor (GR), auch „Typ-2 Rezeptor“ genannt (Myers et al., 2012; Habib et al., 2001). Nur die Glukokortikoidrezeptoren (GR) scheinen größtenteils an der negativen Rückkopplung beteiligt zu sein (Myers et al., 2012; Habib et al., 2001; Charmadari, Tsigos \& Chrousos, 2005). Bei niedrigerer Cortisolkonzentration in den stressfreien Phasen und bei Aufrechterhaltung der zirkadianen Rhythmik erfolgt die Cortisolbindung hingegen an die hochaffinen Mineralokortikoidrezeptoren (MR), auch „Typ-1 Rezeptor“ genannt (Habib et al., 2001; Myers et al., 2012). Die negative Rückkopplung erfolgt an anatomisch unterschiedlichen Regionen des Gehirns, vorwiegend aber bei der Hemmung der $\mathrm{CRH}-$ Sekretion im PVN und in der Hippocampus-Region. Ähnliches gilt für die Region 
im Hypophysenvorderlappen, wo die Freisetzung des ACTH gehemmt wird (z. B. Herman et al. 2016; Lupien et al., 2009; Ulrich-Lai \& Herman, 2009). Der PVN des Hypothalamus mit CRH-Neuronen unterhält Verbindungen zu subkortikalen Bereichen des Großhirns, Thalamus, Amygdala, Hirnstamm, zum Bettkern („,bed nucleus“) der Stria terminalis, zum mPFC, zum NTS sowie zu zahlreichen anderen Hirnregionen und bleibt bei der Aktivierungsphase der HHNA und des Beginns einer Stressreaktion, als auch bei der darauffolgenden Inhibierung derselben maßgeblich beteiligt (Herman et al., 2016; Ulrich-Lai \& Hermann, 2009; Koob, 2008). Im PVN laufen alle Informationen der Stresssysteme zusammen, weshalb ihm die relevanteste physiologische Funktion der zentralen und peripheren Stressorganisation zugeschrieben wird. Die Stressreaktion bei der HHNA erfolgt auf der hormonellen Ebene und ist langsamer als die bei SAM. Sie gilt als „zweite Reaktionsstufe“ bei einer Stressreaktion und hält die notfallbedingte Anpassungsreaktion aufrecht.

Betrachtet man die deutlich unterschiedlichen Formen und Intensitäten der einzelnen Stressoren, erfolgt auch die Cortisolreaktivität hinsichtlich ihrer Konzentration im Blut entsprechend unterschiedlich. Cortisol zirkuliert im Blutkreislauf und entfaltet seine Wirkung bei zahlreichen physiologischen Prozessen und auf unterschiedlichen Ebenen. Beispielsweise werden sympathoadrenerge Prozesse des SAM bei der Freisetzung von Katecholaminen unterstützt, die gespeicherte Energie in Form von Glukoneogenese und Lipolyse mobilisiert sowie die Aktivitäten des Immunsystems unterdrückt (Habib et al., 2001; Ulrich-Lai \& Herman, 2009). Längerfristig kann es bei häufigeren und sehr belastenden Erlebnissen zur stärkeren Aktivierung der HHNA und verstärkten Cortisolstressantwort kommen, was zu einer katabolischen Stoffwechsellage und der Aktivierung proinflammatorischer Zytokine führen kann. Diese stressbedingten Veränderungen stellen Belastungen für den Organismus dar und können die Entwicklung von verschiedenen Erkrankungen beeinflussen, wie 
beispielsweise Krankheiten des Stoffwechsels oder psychische Störungen wie der Alkoholabhängigkeit (McEwen, 2004, Enoch, 2011).

\subsubsection{Determinanten der HHNA-Aktivität}

Die stressbedingten Veränderungen der HHNA-Aktivität, aber auch diese in den stressfreien Phasen, sind für das Überleben eines Menschen wichtig. Es stellt sich die Frage nach den Faktoren, die eine HHNA-Aktivierung beeinflussen. Zur Beantwortung dieser Frage konzentrieren sich die meisten Studien auf Genetik, frühkindliche Entwicklungsbedingungen, Epigenetik und die aktuellen Stressbedingungen. Der genetische Anteil mit multiplen Genvariationen scheint eine bestimmende Rolle zu spielen. So wird der heritabile Anteil des Einflusses auf die HHNA-Aktivität auf $62 \%$ geschätzt (Bartels et al., 2003). Eine vergleichbar starke Wirkung auf die HHNAAktivität scheinen auch die frühkindlichen Lebenserfahrungen auszuüben. Berichtet wird über signifikante Probleme bei Kindern mit psychischen Traumata in der Kindheit.

Diese äußern sich in Form stark veränderter HHNA-Aktivität bei unterschiedlichen physischen und psychischen Erkrankungen (z.B. Heim et al., 2010). Bei nicht wenigen erwachsenen Personen mit kindlichen Traumata wird über abgeschwächte Cortisolreaktion bei Stress berichtet (Carpenter et al., 2011; Lovallo et al., 2012) und mit verlangsamter Rückkehr auf das Niveau vor dem Stress bei alltäglichen Stressbelastungen gerechnet (z. B. Juster et al., 2010). Studien über epigenetische Einflüsse auf die HHNA-Aktivität existieren insbesondere im Zusammenhang mit frühkindlichen Traumata und ihren epigenetischen Auswirkungen auf die HHNAAktivität (Klengel et al., 2013; McGowan et al., 2009). Ferner können jedoch zahlreiche weitere Faktoren die Aktivität der HHNA beeinflussen, wie Geschlecht, Tageszeit, Alkohol, Rauchen, Essgewohnheiten, körperliche Erkrankungen, psychische Störungen, u. v. m. (Übersichtsarbeit bei Kudielka, Hellhammer \& Wüst, 2009). Schließlich unterliegen die HHNA-Aktivität und die Freisetzung von Cortisol einer 
biologischen und zirkadianen Rhythmik mit mehreren Phasen pulsatiler Sekretion (George et al., 2017; Herman et al., 2016; Young et al., 2004).

\subsubsection{Wechselwirkungen zwischen den Stresssystemen der HHNA und SAM}

Die beiden wichtigsten Stresssysteme, SAM und die HHNA, sind in einer hoch komplexen Art und Weise miteinander vernetzt. Sie interagieren miteinander über zahlreiche neuronale Verbindungen, aber auch über Neurotransmitter als Überträgersubstanzen der jeweiligen Stressachsen. Auf peripherer Ebene wird von einer verminderten lipolytischen Aktivität von Adrenalin und Noradrenalin berichtet, wenn die Freisetzung von Cortisol gestört ist (Sapolsky et al., 2000). Im Gehirn steigert Stress die Aktivierung adrenerger Neurone im LC und NTS (SAM-Achse). Über Nervenverbindungen aktivieren diese die neuronale Aktivität im PVN mit der Folge einer $\mathrm{CRH}-$ Freisetzung (HHNA-Achse). Die Ausschüttung von $\mathrm{CRH}$ verstärkt wiederum das LC/-Noradrenalinsystem (SAM-Achse). Für einen Überblick siehe Tsigos et al., 2016.

\subsection{Stress, frühe Stresserfahrungen und Folgen für die Gesundheit: Stress als Initiator und Moderator}

Das Erleben von Stress hängt grundlegend vom Ausmaß der Vulnerabilität und der Resilienz ab. Welche Vulnerabilitätsfaktoren und welche Resilienzeigenschaften bei einer Person vorliegen, wird u. a. durch genetische Prädispositionen, prä-, peri- und postnatale Stresserfahrungen, frühkindliche Schädigungen, Erziehung, Ausbildung, Persönlichkeitseigenschaften, individuellen Lebensstil und die sozioökonomische Situation beeinflusst. Diese Variablen gelten als ein Teil der potenziell gesicherten Risikofaktoren für die Stressvulnerabilität (z. B. Egle, 1997; Wille et al., 2008; Barnes et al. 2009). Sie können die Art und Häufigkeit des Auftretens von Stressoren beeinflussen, aber auch ihre subjektive Bewertung determinieren und damit auch die 
möglichen gesundheitlichen Folgen. Gesundheitsschädliche Verhaltensweisen wie beispielsweise Alkoholmissbrauch, Rauchen und ungesunde Ernährung können indirekt den Stress und die Vulnerabilität verstärken. Auf der anderen Seite können potenziell kompensatorisch wirkende Resilienzfaktoren wie beispielsweise sicheres Bindungsverhalten, dauerhafte gute Beziehung zu mindestens einer primären Bezugsperson, Großfamilie und kompensatorische Elternbeziehungen die pathogene Wirkung der Risikofaktoren reduzieren. Wenn diese empirisch gesicherten Schutzfaktoren hinreichend vorhanden sind, dann können sie die Stressresistenz für die zukünftig belastenden Ereignisse erhöhen (Egle, 2002). Stress ist an der Entstehung und Aufrechterhaltung zahlreicher Störungen beteiligt. Insbesondere Missbrauch, Misshandlung sowie körperliche und emotionale Vernachlässigung in der Kindheit als schwerwiegende stressvolle Erfahrungen können die Vulnerabilität für verschiedene psychische Störungen bedeutend erhöhen (Egle, 2015). Diese Stresserfahrungen erhöhen die Wahrscheinlichkeit, an einer häufigen psychischen Störung zu erkranken wie beispielsweise Depression, Angststörung, Substanzabhängigkeit, um das 2,5- bis 5,5-fache (Kendler et al., 2000). Das kumulative Einwirken der schwerwiegenden frühen Stresserfahrungen hinterlässt sowohl entwicklungspsychologisch als auch neurobiologisch "Narben“ bei der betroffenen Person. In einer sehr groß angelegten und sorgfältig durchgeführten Studie an mehr als 13000 Menschen in Kalifornien konnten Felitti und Mitarbeiter einen Nachweis über das kumulative Einwirken von frühen Stressfaktoren erbringen (Felitti et al., 1998). Sie konnten zeigen, dass das kumulative Einwirken von vier und mehr frühen Stressfaktoren in der Kindheit im Vergleich zu deren vollständigem Fehlen zu einem 4- bis 12-fach erhöhtem Risiko für Alkoholismus, Drogenmissbrauch, Depression und Suizidversuch führt. Diese Verhaltensweisen können als dysfunktional-ineffektive Bewältigungsversuche bewertet werden, beispielsweise 
niedriges Selbstwertgefühl, aber auch die erhöhte Stressanfälligkeit zu kompensieren. Anhaltender Stress oder sich häufig wiederholende Stresseinwirkungen führen im Laufe des Lebens über erhöhte Konzentration an Glukokortikoiden zu Schädigungen am Hippocampus und damit einhergehenden Störungen im Kurzzeitgedächtnis (Lupien et al., 1998). Die in diesem Zusammenhang erhöhte Konzentrationen insbesondere an Noradrenalin, aber auch Dopamin, führen zu Schädigungen der Amygdala-Kerne (Roozendaal, et al., 2009). Die Schädigungen im Hippocampus haben ihre Konsequenzen für die weitere Freisetzung des Cortisols bzw. sorgen für die Störungen bei der negativen Rückkopplung (Roozendaal, 2009). Hinzu kommen Störungen bei der assoziativen Verknüpfung von Erlebnisinhalten.

Im Erwachsenenalter ist der Stress ebenfalls an der Entstehung und Aufrechterhaltung zahlreicher Erkrankungen beteiligt. Nach einem einschneidenden Lebensereignis erleben Menschen häufig ihre erste depressive Episode. Kausale Zusammenhänge zwischen einschneidenden Lebensereignissen und der Depression gelten schon längst als nachgewiesen (z. B. Hammen, 2005; Kendler et al., 1999). Das Vorliegen einer schweren medizinischen Erkrankung wird häufig als schwerer Stressor bewertet und geht nicht selten mit einer Depression einher. Beispielsweise wurden in einer Metaanalyse bei Krebspatienten hohe Raten an schweren depressiven Episoden diagnostiziert (Übersicht bei Massie, 2004). Chronischer Stress steht im Zusammenhang mit verlangsamter Wundheilung und Erholung von der Operation, abgeschwächten Antikörperreaktionen auf Impfungen und reduzierter Immunität auf virale Infekte (Kiecolt-Glaser et al., 2002). Bei kardiovaskulären und Atemwegserkrankungen gibt es ebenfalls Hinweise für eine Beteiligung des Stresses an der Entstehung und Aufrechterhaltung (Kemeny \& Schedlowski, 2007; Krantz \& McCeney, 2002; Cohen et al., 1998). Bei Grippe beispielsweise verursachen 
proinflammatorische Zytokine Symptome wie Müdigkeit, Antriebslosigkeit, Appetitlosigkeit, die bei einer Depression auch vorkommen. Die proinflammatorischen Zytokine können aber auch, in Abwesenheit einer Infektion, Krankheitsverhalten („sickness behavior“) wie beim Vorhandensein einer solchen verursachen (Dantzer, 2001). Allerdings gibt es zunehmende Beweise dafür, dass psychologische Stressoren, vermittelt über einen ähnlichen Mechanismus, ebenfalls „sickness behavior“ auslösen können (Simmons \& Broderick, 2005). Akuter psychologischer Stress führt zu einem Anstieg von proinflammatorischen Zytokinen und Symptomen negativer Stimmung bei gesunden Personen (Steptoe et al., 2007). Obwohl Stress immunsuppressive Effekte verursacht, wird er interessanterweise mit der Entstehung von Autoimmunkrankheiten sowie anderen klinischen Bildern, bei denen verstärkte Entzündungen zentrales klinisches Merkmal darstellen, trotzdem in Verbindung gebracht (Kemeny \& Schedlowski, 2007; Harbuz et al., 2003).

\subsection{Burnout}

Unter dem Begriff „Burnout“ wurde in den letzten Jahren vermehrt über eine Zunahme von psychischen Störungen berichtet, die wiederum im Zusammenhang mit belastenden Arbeits- und Lebensbedingungen stehen (Hall et al., 2016). Der Begriff geht auf den Psychoanalytiker Herbert J. Freudenberger zurück, der 1974 erstmals Burnout als Zustand ausführlich beschrieb (zitiert nach Schaufeli, 2017). Freudenberger beschrieb den psychophysischen Abbau der Mitarbeiter von psychosozialen Hilfsorganisationen. Damals handelte es sich meist um ehrenamtliche Mitarbeiter, die als Idealisten mit unrealistisch hohen altruistischen Zielen arbeiteten. Heutzutage sind solche Mitarbeiter eher die Ausnahmen (Farber, 2000). Das typische Burnout-Profil sieht heute ganz anders aus und wird von dem sozioökonomischen Druck bestimmt, „immer größer werdende Ansprüche anderer zu erfüllen, oder sich 
der Konkurrenz zu stellen, besser als andere in einem Unternehmen zu sein, oder angetrieben zu sein, immer mehr Geld zu machen“, ... usw. (Farber, 2000). Beim Burnout handelt es sich nicht um ein umschriebenes Krankheitsbild nach der ICD-10. Es ist also keine eigenständige psychische Diagnose. In der ICD-10 wird Burnout als „Ausgebranntsein“ und „Zustand der totalen Erschöpfung“ mit dem Diagnoseschlüssel Z73.0 erfasst. Das bedeutet, dass es sich um einen Faktor handelt, der den Gesundheitszustand beeinflusst und zur Inanspruchnahme des Gesundheitswesens führt. Eine Z-Verschlüsselung darf nach ICD-10 nur im Zusammenhang mit anderen Diagnosen verwendet werden. Das Burnoutsyndrom ist als Konstrukt sehr fragil mit einer inkohärenten Struktur und weist sehr große Überlappungen mit der Depression auf (Bianchi et al., 2015). Aus diesen Gründen wird dieses Konstrukt hier nur in kurzen Zügen erwähnt. Die Frage stellt sich aber trotzdem, warum ein Burnout auftritt? Wodurch wird die Balance zwischen Anforderungen in der Lebenswelt und der Bewältigungskompetenz gestört? Ist es vielleicht eine Folge von Vulnerabilität und der Betroffene kommt rasch an seine Grenzen, weil die Bewältigungsressourcen schnell erschöpft sind? Diese und weitere Fragen im Bereich der Stressbelastung werden wahrscheinlich Gegenstand zukünftiger klinischer Forschungsbemühungen sein.

\subsection{Stress und Resilienz}

Wie oben schon erwähnt, existieren potenziell protektiv oder kompensatorisch wirksame Faktoren, die vor Stresseinwirkung „schützen“ und die Vulnerabilität für belastende Erfahrungen mildern. Der Begriff „Resilienz“ hat in den letzten Jahren ebenfalls an Bedeutung gewonnen. Es bezieht sich auf die Fähigkeit einer Person, sich an akuten Stress oder chronische Belastungen anzupassen (Feder et al., 2009). In einer sehr ansprechenden Übersichtsarbeit werden von Feder und Mitarbeitern mehrere psychosoziale Faktoren genannt, die als psychobiologisch und 
molekulargenetisch untermauert mit Resilienz assoziiert sind (Feder et al., 2009). Es werden Faktoren genannt in etwa wie: sich der Angst sowie den Herausforderungen zu stellen und aktive Bewältigung dieser, Optimismus und positive Gefühle, kognitive Neubewertung, Neuausrichtung und Akzeptanz, soziale Kompetenz und soziale Unterstützung, Lebenssinn, moralischer Kompass, Sinn und Spiritualität. Betont wird, dass Resilienz kein statischer Zustand und keine Abwesenheit von somatischen Symptomen oder Psychopathologie ist. Sie ist mehr ein aktiver Prozess, den man durch die Verbesserung von günstigen Verhaltensweisen und Lebensbedingungen (Schutzfaktoren) fördern kann (Feder et al., 2009). Studien zeigen, dass Menschen widerstandsfähiger werden können. Als Beispiel wird die psychotherapeutische Behandlung, insbesondere die kognitive Verhaltenstherapie, als Methode erwähnt, die den Optimismus verstärkt und durch die Neubewertung der stressigen Ereignisse, diese in einem neutraleren oder sogar positiveren Licht erscheinen lassen (Feder et al., 2009).

\subsection{Stressinduktionsmethoden}

Nachdem das Phänomen Stress mit unterschiedlichen Stresskonzepten vorgestellt und die Stressreaktion mit den beiden wichtigsten Stresssystemen, SAM und HHNA, erläutert wurde, werden im nächsten Abschnitt und in kurzen Zügen Methoden der Stressinduktion vorgestellt. In der veröffentlichten Originalarbeit wird zwar auf Stressinduktion eingegangen, aber nur in einem notwendig kurzen Format.

Generell existieren zahlreiche Stressoren physischer und psychischer Natur, die in der Lage sind, eine Stressreaktion zu erzeugen. Je nach Eigenschaft können sie unterschiedlich eingeteilt werden. Hinsichtlich der Wirkungsdauer eines Stressreizes können sie in akute und chronische Stressoren eingeteilt werden. Bei der Durchsicht wissenschaftlicher Literatur zeigt sich hinsichtlich stressinduzierender Methoden eine 
weitgehende Aufteilung in zwei Gruppen. In der ersten Gruppe werden Stressoren vorgestellt, die unter wirklichkeitsgetreuen Lebensbedingungen eine Stressreaktion erzeugen und können unter dem Oberbegriff der lebensnahen naturalistischen Stressoren zusammengefasst werden. Ein prominentes Beispiel für diese Stressorengruppe ist die Leistungsbeurteilung in der Schule oder einer Prüfungssituation. Dabei konnten unmittelbar vor der Prüfung signifikant stärkere Cortisolstressantworten bei den Prüfungskandidaten im Vergleich zu Kontrollgruppe beobachtet werden (z. B. Martinek et al., 2003).

Die zweite Stressorengruppe wird unter dem Oberbegriff Laborstressoren zusammengefasst. Unter kontrollierten Bedingungen bietet sich die Möglichkeit, unter Darbietung verschiedener Stressreize eine Stressreaktion im Labor zu erzeugen. Die Stressreize können in Form von Schmerz, Kälte, Hitze oder eines Medikamentes dargeboten werden. Weil sie meist direkt auf die Regionen im Hypothalamus Einfluss nehmen, haben sie somit entsprechend einen direkten Einfluss auch auf die Physiologie im Organismus (Hermann \& Cullinan, 1997). Einen bekannten Vertreter aus dieser Gruppe stellt der Cold Pressor Test (CPT) dar. Bei diesem Verfahren sollen die Teilnehmer, so lange sie es tolerieren können oder bis drei Minuten, ihre dominante Hand in ein eiskaltes Wasser $\left(0-1{ }^{\circ} \mathrm{C}\right)$ eintauchen (Velasco et al., 1997). Der CPT kann über die Thermo- und Schmerzrezeptoren eine Stressreaktion von beiden Stresssystemen auslösen, auch bei Alkoholabhängigen (Brady et al., 2006). Neben den Laborstressoren, die auf eine direkte Einwirkung auf physiologische Prozesse abzielen, existieren noch psychologische Stressoren. Die Stresssituation kann auch durch Induktion von Emotionen mithilfe von Filmen oder Bildern erzeugt werden. Die am häufigsten eingesetzten Verfahren sind aber diejenigen, bei denen situative Stressbedingungen so konstruiert werden, dass der Proband einer kritischen 
Beurteilung durch andere Personen ausgesetzt wird. Der effektivste und am weitesten verbreitete Test unter diesen ist der Trier Sozial Stress Test (TSST; Kirschbaum et al., 1993). Er gilt als zuverlässiger Aktivator der beiden Stresssysteme SAM und HHNA (Dickerson \& Kemeny, 2004). Unabhängig von manchen kritischeren Stimmen (Allen et al., 2014) gilt der TSST als „Goldstandard“ unter den Stressoren mit sozialer Interaktion (Thomas et al., 2012). Der Test wird nach einer standardisierten Versuchsanordnung durchgeführt. Er besteht, vereinfacht dargestellt, aus einem in freier Rede geführten Bewerbungsvortrag und einer anspruchsvollen Rechenaufgabe. Beide Aufgaben dauern jeweils fünf Minuten und werden vor einem aus mehreren Personen bestehenden Gremium durchgeführt. Die Probanden werden instruiert, dass sie sich so überzeugend wie möglich vor diesem sie bewertenden und in der Verhaltensanalyse geschultem Gremium präsentieren sollten. In den Forschungsarbeiten zum TSST werden die wichtigsten Aspekte erwähnt, die eine effektive Stressreaktion auslösen. Dazu zählt vor allem die subjektive Wahrnehmung der „Bedrohlichkeit“ unter sozialer Beurteilung wichtiger eigener Identitätselemente. Hinzu kommen auch die Neuheit und Unkontrollierbarkeit der Stresssituation, bzw. keine Möglichkeit zu haben, den Ausgang der Situation zu beeinflussen (Dickerson \& Kemeny, 2004). Bei der Cortisolstressantwort bestehen aber interindividuelle Unterschiede (Kudielka \& Kirschbaum, 2005; Kudielka et al., 2007), für deren Ursachen genetische Determinanten vermutet werden (Federenko et al., 2004). Bei wiederholter Durchführung reagieren die meisten Probanden mit einer abgeschwächten HHNA-Aktivität bzw. mit Habituation (Schomer et al., 2003). In der eigenen Studie reagierten alle Probanden auf TSST mit Erhöhung des Cortisols, weshalb seine Anwendung als erfolgreich bezeichnet werden kann (Tolic \& Soyka, 2018). 


\subsection{Erfassungen biologischer Variablen der Stressreaktion (Cortisol, EDA und Puls)}

\subsubsection{Cortisol}

Das Hormon Cortisol kann als physiologisches Bindeglied zwischen den neuronalen Prozessen im ZNS einerseits und physiologischen und endokrinologischen Prozessen der Peripherie andererseits angesehen werden. Cortisol ist der bekannteste Vertreter der Glukokortikoide und diese wiederum sind bekannteste Vertreter der Steroidhormone. Glukokortikoide sind hochgradig lipophil und können problemlos sowohl eine Zellmembran als auch die Blut-Hirn-Schranke passieren (Mason et al., 2010; Mason et al., 2008). Die Freisetzung von Glukokortikoiden wird durch den Glukokortikoid-Rückkopplungsmechanismus bei verschiedenen Aktivitätsgraden der HHNA kontrolliert (Myers et al., 2012). Diese Regulierung ist von großer Relevanz, weil zu hohe Cortisol-Konzentrationen zu pathologischen Veränderungen an verschiedenen Organen und Organsystemen führen können (Myers et al., 2012). Das ACTH wirkt an der „Zona fasciculata“ der Nebennierenrinde an Melanocortin-2Rezeptoren (MC2R). Dies führt zur Erhöhung des intrazellulären cyclischen Adenosinmonophosphat-Spiegels (cAMP), was einen schnellen Anstieg der Cholesterin-Biosynthese zur Folge hat (Hadley \& Haskell-Luevano, 1999). Das Cholesterin ist bekanntlich die Vorstufe von den meisten Steroiden und somit auch der Glukokortikoide. Cortisol wird also in der inneren Zone, auch „zona fasciculata“ genannt, der Nebennierenrinde gebildet. In den weiteren zwei Zonen der Nebennierenrinde, der „zona reticularis“ und der „zona glomerulosa“ werden Androgene und Mineralokortikoide produziert (Schandry, 2016; Pape et al., 2014). Es wird in mehreren Phasen pulsatil in den systemischen Blutkreislauf freigesetzt (George et al., 2017). Abgesehen von den situativen Schwankungen und 
interindividuellen Unterschieden zeigen die Cortisolwerte einen spezifischen Tagesverlauf (Clow, 2004; Pruessner et al., 1997; Adam \& Kumari, 2009). In der Nacht befinden sich die Werte auf einem Minimum, steigen aber in der zweiten Nachthälfte bis in die frühen Morgenstunden kontinuierlich an und sind 30-45 Minuten nach dem Erwachen an ihrem Maximum angelangt (Clow, 2004; Adam \& Kumari, 2009). Dieser normale Biorhythmus des Cortisols wird durch Fehlregulation der HHNA gestört (Clow, 2004; Clow et al., 2010; de Kloet, 2004). Wird der TSST als Stressinduktionsmethode verwendet, erreicht das Speichelcortisol sein Maximum erst 10-20 Minuten nach Beendigung des TSST-Paradigmas (Kudielka et al., 2007). Eine Erhöhung des Speichelcortisols von $2,5 \mathrm{nmol} / \mathrm{l}$ im Vergleich zum individuellen Basiswert eines Probanden wird als signifikanter Anstieg gewertet (Wüst et al., 2005). Für die laborchemischen Analysen kann das Cortisol aus Blut, Speichel oder Urin (als 24-h-Sammelurin) gewonnen werden. Eine valide und zuverlässige ebenso praxistaugliche Möglichkeit zur Beurteilung der HHNA-Aktivität ist die Bestimmung des Speichelcortisols (Hellhammer et al., 2009; Kirschbaum \& Hellhammer, 1994; Kirschbaum, 1991). Diese Methode ist in der Stressforschung beliebt und hat sich seit mindestens 20 Jahren sehr gut etabliert (Ryan et al., 2016). Nur etwa 5-15\% des im systemischen Blutkreislauf freigesetzten Cortisols ist ungebunden und somit in physiologisch aktiver Form (z. B. Gozansky et al., 2005; Aardal-Eriksson et al., 1998; Kirschbaum \& Hellhammer, 1994). Der Rest ist an Globuline und Albumine im Plasma gebunden. Obwohl es keine Richtlinien zur Messung des Speichelcortisols gibt, sollten bei der Bestimmung eines Tagesprofils mindestens drei Messungen erfolgen (Ryan, 2016). Seine Zuverlässigkeit als Parameter bestätigt Cortisol sowohl bei der Gewinnung aus dem Serum als auch aus dem Speichel, wobei das Speichelcortisol als physiologisch aktiv zirkulierendes Cortisol eine genauere Beurteilung der HHNAAktivität erlaubt (Gozansky et al., 2005). Die im Labor nachweisbaren Effekte finden 
sich vergleichsweise nicht im gleichen Umfang in den Feldstudien. Im Vergleich mit Laborsettings stehen bei solchen Studien weniger die kurzfristigen Cortisolstressantworten im Vordergrund als vielmehr das oben erwähnte Cortisoltagesprofil. Diese Forschungsbreite gibt dem Cortisol eine weitreichende Bedeutung. Diese wird in einer sehr anschaulichen Metaanalyse von 107 Studien eindrucksvoll bestätigt (Miller et al., 2007). Als zuverlässige Ergebnisparameter für die HHNA-Aktivität bei Alkoholabhängigen werden ACTH und Cortisol häufig bei Querschnittsstudien verwendet (Thomas et al., 2012). So auch das Cortisol bei der eigenen Studie. Bei Langzeitstudien mit Alkoholabhängigen ebenso (Ruttle et al., 2015).

\subsubsection{Elektrodermale Aktivität (EDA) und Puls}

Elektrodermale Aktivität (EDA) und Puls gelten als zuverlässige Parameter bei der Beurteilung der SAM-Achse (z. B. Davson et al., 2007; Sher et al., 2007; Brownley et al., 2000). Obwohl Puls, Herzratenvariabilität und die EDA-Messung in der Stressforschung weit verbreitet sind, dominiert die letztere auch noch in der Angstforschung (Phelps \& LeDoux, 2005). Beide Parameter haben mehrere gemeinsame neuronale Repräsentanzen, die vorwiegend im ventromedialen präfrontalen Cortex (wmPFC), temporal parahippocampalen Cortex, Thalamus und Hirnstamm lokalisiert sind (Eisenbarth et al., 2016). Bei der Darbietung von sozial bedrohlichen Reizen gibt es Hinweise darauf, dass diese keine einheitlichen sympathoadrenergen, sondern multiple Stressreaktionen in verschiedenen Körpersystemen hervorrufen (Eisenbarth et al., 2016). Beide Parameter, EDA und Puls, sind relativ einfach und schmerzfrei für die Versuchsperson erfassbar. Während der Durchführung von TSST wird durch die erlebte situative „Bedrohlichkeit“ eine hohe 
psychische und physiologische Erregung erzeugt, die den Sympathikus aktiviert. Dieser erzeugt eine starke Herzaktion und bewirkt eine stärkere Schweißproduktion in den Schweißdrüsen. Durch die erhöhte Schweißproduktion reduziert sich der Leitungswiderstand der Haut, währenddessen sich die Hautleitfähigkeit erhöht (Schandry, 2016). Die Schweißdrüsen unterliegen einer direkten Kontrolle des autonomen Nervensystems. Bei der EDA soll es sich um einen relativ genauen Parameter für die Evaluierung der SAM-Aktivität bei emotionalen Vorgängen handeln (Critchley, 2002).

Auf erhöhte elektrodermale Reaktivität bei Alkoholabhängigen wird in einer Studie von Demmel und Mitarbeitern hingewiesen (Demmel et al., 2000). In einer neueren Studie mit 32 abstinent lebenden Alkoholikern wurden abgeschwächte Reaktionen der EDA und Puls auf emotionale Reize im Vergleich zu gesunden Probanden beobachtet (Sawyer et al., 2015). Bei einer Studie mit Opiatabhängigen mit Stressparadigma (TSST) konnte eine signifikant stärkere Stressreaktion in Form von erhöhten Werten für Cortisol, Puls und EDA beobachtet werden (Back et al., 2015). Bei der eigenen Studie mit Alkoholabhängigen konnte ein ähnliches Ergebnis für schwerkranke Patienten gefunden werden (Tolic \& Soyka, 2018).

\section{Erhebungsinstrumente: Diagnostik und Psychopathologie}

In der durchgeführten Studie wurden mehrere standardisierte Erhebungsinstrumente verwendet. Zwei Tests, EuropASI und SESA, wurden bereits im Kapitel 2.4 zum Thema Schweregrad der Alkoholabhängigkeit vorgestellt. An dieser Stelle sollen die restlichen Testverfahren kurz vorgestellt werden, die in der Studie eingesetzt waren. 


\subsection{AUDIT-Fragebogen (Alcohol Use Disorders Identification Test)}

Es handelt sich um einen Selbstbeurteilungstest, bestehend aus zehn Fragen hinsichtlich der Trinkmenge, Trinkdauer und den Trinkgewohnheiten, entstanden in den 80er Jahren durch die Arbeitsgruppe um Thomas F. Babor auf Anregung der WHO. $\mathrm{Zu}$ jeder der zehn Fragen kann ein Punktwert zwischen 0 und 4 Punkte gewählt werden und somit eine Punktzahl zwischen 0 und 40 erreicht werden. Als Cut-off für einen Verdacht auf alkoholbezogene Störung gilt ein Wert von acht Punkten (WHO). Mit höherem Scorewert steigt die Wahrscheinlichkeit, dass bei dem Betroffenen eine Alkoholabhängigkeit vorliegt. Ein kritischer Wert liegt zwischen 15 und 20 Punkten. Der AUDIT-Fragebogen wird als zuverlässiges Screening-Instrument zur Diagnostik von alkoholbezogenen Störungen angesehen (z. B. Isaacson et al., 1994; Aertgerts et al., 2001).

\subsection{Mannheimer Craving Scale (MaCS)}

Dient der quantitativen Erfassung von Suchtdruck verschiedenster Substanzen. Wurde von der Arbeitsgruppe um Helmut Nakovics und Karl Mann entwickelt (Nakovics et al., 2009). Der Test besteht aus 12 Items und weiteren vier Zusatzitems. Zu jeder der 12 Fragen stehen fünf Antwortmöglichkeiten von 0 bis 4 zur Verfügung. Die vier Zusatzitems erfragen in drei visuellen Analogskalen die Intensität und die Häufigkeit des Cravings in den letzten sieben Tagen und ein Item erfasst die Abstinenzdauer. Durchs Addieren der ersten 12 Items erhielt man den MaCS-Score. Der Fragebogen hat hohe Messzuverlässigkeit und ist in der klinischen Forschung und Praxis gut anwendbar (Nakovics et al., 2009). In der durchgeführten Studie zeigten schwerkranke Patienten stärkeren Suchtdruck im Vergleich zu der Gruppe der leichtmittelschwer Kranken. 


\subsection{Stresstests}

\subsubsection{Der Stressverarbeitungsfragebogen 120 (SVF 120)}

Der Stressverarbeitungsfragebogen (Erdmann \& Janke, 2008) kann als eines der etabliertesten und im deutschsprachigen Raum am häufigsten eingesetzten Verfahren zur Erfassung der Stressverarbeitung gelten. Er besteht aus 120 Items, die in 20 Subskalen aufgeteilt sind. Diese sind: Bagatellisierung, Herunterspielen, Schuldabwehr, Ablenkung von Situationen, Ersatzbefriedigung, Selbstbestätigung, Entspannung, Situationskontrolle, Reaktionskontrolle, Positive Selbstinstruktion, Soziales Unterstützungsbedürfnis, Vermeidung, Flucht, Soziale Abkapselung, Gedankliche Weiterbeschäftigung, Resignation, Selbstbemitleidung, Selbstbeschuldigung, Aggression und Pharmakaeinnahme. Der Test erlaubt eine übergeordnete Auswertung in „Positiv-Strategien“ und „Negativ-Strategien“ der Stressverarbeitung. Die Auswertung erfolgt für jede Subskala getrennt und die Ergebnisse werden in einem Profil festgehalten. Die Normierung nach T-Werten sieht eine Punktzahl zwischen 40 und 60 als normal vor. Eine Punktzahl zwischen 20 und 40 gelten als unterdurchschnittlich und zwischen 60 und 80 als überdurchschnittlich. Der Fragebogen weist gute Testgütekriterien auf.

\subsubsection{Trierer Inventar zum chronischen Stress (TICS)}

Das Trierer Inventar zum chronischen Stress (TICS) von Schulz, Schlotz und Becker (2004) ist ein Fragebogen, der das subjektive Stresserleben auf der Basis komplexer Stresserfahrungen und aus heterogenen Belastungsquellen transparent erfasst. Es besteht aus 57 Items, die unterschiedliche chronische Stressbereiche abbilden und zehn Skalen zugeordnet sind. Diese sind: Arbeitsüberlastung, Soziale Überlastung, Erfolgsdruck, Unzufriedenheit mit der Arbeit, Mangel an sozialer Anerkennung, soziale Spannungen, soziale Isolation, chronische Besorgnis und die Screening-Skala zum 
chronischen Stress (SSCS), die mit 12 Items die chronische Stressbelastung global erfasst. Mit guten Testgütekriterien ist das TICS ein zentrales und zuverlässiges Instrument zur Messung chronischer Stressbelastung (Ditzen \& Nater, 2006).

\subsubsection{Inventar zur Erfassung Lebensverändernder Ereignisse (ILE)}

Beim ILE handelt es sich um ein Testverfahren, welches lebensverändernde Ereignisse der letzten zwei Jahre und das subjektive Erleben dieser erfasst. Es wurde 1981 von Dittman und Siegrist, später aber von Siegrist und Geyer weiterentwickelt (Siegrist \& Geyer, 2002). Es zeigt hohe Ähnlichkeiten mit der Social Readjustment Rating Scale (Holmes \& Rahe, 1967). Der ILE-Fragebogen erfasst, ob und wie oft ein Lebensereignis in den letzten zwei Jahren bei der zu untersuchenden Person aufgetreten ist. Pro Lebensereignis wird ein Belastungswert berechnet und aus allen Belastungswerten wird pro Person ein Gesamtbelastungssummenwert gebildet. Die Objektivität und die Validität als Testgütekriterien werden als erfüllt beschrieben (Siegrist \& Geyer, 2002).

\subsubsection{Alltagsbelastungsfragebogen (ABF)}

Der Test wurde von Harald Traue und Mitarbeitern entwickelt (Traue et al., 2000). Er wird häufig als die deutsche Version des amerikanischen Fragebogens Daily Stress Inventory (Brantley et al., 1987) betrachtet. Der ABF besteht aus 58 Items und erhebt die Häufigkeit, mittlere Belastung sowie den Summenwert von alltäglichen negativen Stressoren in den letzten 24 Stunden. Die Testgütekriterien werden als befriedigend angegeben und der Test insgesamt als geeignet für die Erfassung von alltäglichen Stressbelastungen beschrieben (Traue et al., 2000). 


\subsection{Psychopathologie}

\subsubsection{State-Trait-Angstinventar (STAI) und Becks-Depressions-Inventar II (BDI-II)}

In der eigenen Studie wurde die Angst als Variable mit dem State-Trait-Angstinventar in der deutschen Version von Laux und Mitarbeiter erhoben (Laux et al., 1981). Die Originalversion wurde 1970 von Spielberger und Mitarbeitern entwickelt (Spielberger et al., 1970). Der Fragebogen besteht aus zwei getrennten Skalen, die jeweils Zustandsangst (State-Angst) und habituelle Angst (Trait-Angst) erfassen. Jede Skala enthält jeweils 20 Feststellungen mit unterschiedlichen Intensitätsgraden. Der STAI wird seit vielen Jahren in Forschung und Praxis als zuverlässiges und etabliertes Instrument zur Erfassung von Ängsten verwendet. Die Testgütekriterien werden weitgehend als akzeptabel beurteilt (Barnes et al., 2002; Laux et al., 1981).

Die Depression wurde mittels Becks-Depressions-Inventar-II (BDI-II) erhoben. Es wurde ursprünglich entwickelt in den 80er Jahren von Beck und Mitarbeitern (Beck et al., 1961, zitiert nach Schmitt et al., 2010). In der vorliegenden Studie wurde die deutsche Version von Hautzinger und Mitarbeitern verwendet (Hautzinger et al., 1994, aktuelle Version Hautzinger et al., 2009). Beim BDI handelt es sich um ein sehr zuverlässiges und seit über 30 Jahren breit anwendbares Testverfahren zur Erfassung von Depression (Herzberg et al., 2008, Schmitt et al., 2010). Die Auswertung ist sehr einfach. Den einzelnen Items werden Werte zwischen 0 und 3 Punkten zugeordnet. Es können Summenwerte zwischen 0 und 63 Punkten erreicht werden.

\section{Strukturiertes Klinisches Interview für DSM-IV (SKID-II)}

Das Vorliegen einer Persönlichkeitsstörung (PS) in der Allgemeinbevölkerung wird hinsichtlich der Prävalenz auf 7-15\% geschätzt (Johnson et al., 2008). Ebenso häufig wird von einer hohen Komorbidität mit Alkoholabhängigkeit berichtet (Preuss et al., 2009). Aus diesen Gründen sollten diagnostische Bemühungen bei klinischen 
Untersuchungen eine Diagnostik von Persönlichkeitsstörungen beinhalten. In der vorliegenden Studie wurde das Strukturierte Klinische Interview für DSM-IV (SKID-II) verwendet (SKID-II; Wittchen et al., 1997). Beim SKID-II handelt es sich um ein zweiteiliges Testverfahren, das aus einem Fragebogenteil und einem Interviewteil besteht. Die 117 Items des Fragebogens werden vom Probanden ausgefüllt und erfassen 12 Persönlichkeitsstörungen. Danach wird das Interviewheft eingesetzt, um die bejahten Fragen mit dem Probanden zu bearbeiten und zu prüfen, ob die Kriterien für eine PS erfüllt werden oder nicht. Bei der Auswertung der Antisozialen Persönlichkeitsstörung werden lediglich Kriterien vor dem 15. Lebensjahr berücksichtigt. SKID-II gehört im klinischen Alltag zum Standardverfahren bei der Diagnostik von PS. Dem SKID-II werden gute Testgütekriterien zugeschrieben und es wird als „Goldstandard“ in der Diagnostik von PS bezeichnet (Smith et al., 2003).

An dieser Stelle erfolgt die Vorstellung der eigenen veröffentlichten Studie (Tolic \& Soyka, 2018) in ihren wichtigsten Teilbereichen, nämlich Methodik, Ergebnisse und die Diskussion.

\section{Methoden}

Die Zusammenhänge zwischen Alkoholabhängigkeit und Stress sind hochkomplex und nicht endgültig geklärt. Insbesondere die genauen Muster der wechselseitigen Beziehung zwischen unterschiedlichen Schweregraden der Alkoholabhängigkeit und der Stressreagibilität sind immer noch unklar. Das Gleiche gilt hinsichtlich der Abstinenzdauer.

\subsection{Ziel der Studie}

Die vorliegende Arbeit prüfte, ob neben der Abstinenzdauer auch die Krankheitsschwere bei der Stressreaktion eine Rolle spielt. Sie untersuchte, ob und in 
inwiefern sich Alkoholabhängige sowohl untereinander als auch von den gesunden Probanden im Hinblick auf unterschiedliche Reaktionsformen wie neuroendokrinologische, stressphysiologische sowie psychopathologische und testpsychologsiche Variablen unterscheiden. Ein weiteres Ziel bestand darin zu prüfen, ob und inwiefern sich Alkoholabhängige in ihrem Stressbewältigungsstil untereinander als auch von den gesunden Probanden unterscheiden. Ein weiteres Ziel war auch mehr Klarheit in der hochkomplexen bidirektionalen Beziehung zwischen Alkoholabhängigkeit und Stress zu gewinnen, die für die Entwicklung individueller Behandlungsansätze nützlich sein könnten. Diese Kontrollgruppenstudie im Querschnittdesign wurde von der Ethikkommission der Ludwig-MaximiliansUniversität genehmigt.

\subsection{Stichproben}

Insgesamt wurden 66 alkoholabhängige Patienten und 30 gesunde Probanden untersucht. Die Daten von zwei Alkoholabhängigen waren unvollständig und konnten nicht ausgewertet werden. Die Stichprobe der Alkoholabhängigen bestehend aus 64 Personen wurde hinsichtlich Abstinenzdauer und Schweregrad der Abhängigkeit in jeweils zwei Gruppen aufgeteilt. Durch die Aufteilung der Patienten in Untergruppen nach Abstinenzdauer und Krankheitsschwere wird ein guter Vergleich zwischen einer pathogenetischen Wirkung der Alkoholkrankheit auf Stressreagibilität und Stressverarbeitung möglich. Im Bezug auf die Abstinenzdauer wurden die Patienten in zwei Gruppen aufgeteilt. Die erste Gruppe mit 31 Patienten bildeten Probanden, die eine Alkoholabstinenz von sieben Tagen aufweisen (Gruppe A7). Die zweite Gruppe bestand aus 33 Patienten und wies eine Abstinenzdauer von 70 Tagen auf (Gruppe A70).

Die Aufteilung nach Schweregrad der Abhängigkeit wurde Anhand der erfassten Daten in den Fragebögen EuropASI und SESA durchgeführt (s. Abschnitt 
Schweregrad d. Abhängigkeit). Die beiden Instrumente, EuropASI und SESA, ermöglichen eine Aufteilung im Hinblick auf Krankheitsschwere in jeweils zwei Gruppen. Die Gruppe „leicht bis mittelschwer“ und „schwer“. So konnten von den 64 Patienten der Gesamtstichprobe, 35 Patienten als leicht-mittelschwer und 29 als schwer alkoholabhängig identifiziert werden.

Die Stichprobe der gesunden Probanden bestand aus 30 Personen (17 Männer und 13 Frauen) und ist mit der Patientengruppe (36 Männer und 28 Frauen) im Hinblick auf soziodemographische Merkmale (Alter, Schulbildung, usw.) vergleichbar $(p=.36$ und $p=.56$ ). Die durchschnittliche Schulbildung bei Alkoholabhängigen betrug 13,4 Jahre (SD \pm 2,3 Jahre). Knapp über die Hälfte der Patienten (52\%) lebte in einer festen Beziehung. Alleine lebten 35\% der Patienten. Mehr als die Hälfte der Patienten (59\%) waren berufstätig als Angestellte, Arbeiter oder Selbständige. Dauerhaft arbeitslos waren $32 \%$ der Patienten.

\subsection{Untersuchungsablauf}

Der Untersuchungsablauf wurde so konzipiert, dass alle Probanden in den gleichen Räumlichkeiten und zur gleichen Tageszeit untersucht worden sind. Für die jeweiligen Stichproben wurden Ein- und Ausschlusskriterien festgelegt (Kasten 1). Somit konnten viele Faktoren ausgeschlossen werden, die als kritische Einflussgrößen im Bezug auf eine Stressreaktion gelten. Insbesondere war es wichtig, die Stimulation der HHNA und die Ausschüttung von Cortisol vor der Untersuchung zu verhindern. Es gab z. B. keine Blutentnahmen. Zunächst wurde mit jedem Probanden ein Interview durchgeführt, in dem soziodemographische sowie andere relevante Daten erhoben wurden. Der Patientenfragebogen erfasste die wichtigsten demographischen Variablen, Krankheitsvorgeschichte, Trinkdauer, Trinkmenge sowie alle anderen gesundheitsbezogenen Variablen. Vor der Testung mussten 
Alkoholabhängige ärztlich untersucht worden sein und einen Nachweis für die Alkoholabstinenz erbringen. Vorwiegend konnten ihre Blutwerte vom stationären Aufenthalt, nicht älter als sieben Tage, genutzt werden. Alle Probanden mussten sich der Testung des Alkoholgehalts in der Atemluft (Alcomat) unterziehen. Die Stressinduktion erfolgte mittels Trier Sozial Stress Test (TSST) und die Stressreaktionen wurden anhand von Cortisolproben, Puls und elektrodermaler Aktivität (EDA) erfasst.

\begin{tabular}{|c|c|c|}
\hline Stichprobe & Einschlusskriterien & Ausschlusskriterien \\
\hline Alkoholabhängige & $\begin{array}{l}\text { Diagnose } \\
\text { Alkoholabhängigkeit, } \\
\text { Alter 20-65 Jahre, } \\
\text { Einverständnis zur } \\
\text { Teilnahme an der } \\
\text { Studie, ärztliche } \\
\text { Untersuchung, } \\
\text { Laborwerte. }\end{array}$ & $\begin{array}{l}\text { Schwere abhängigkeitsassoziierte Folgeerkrankungen (z. B. } \\
\text { dekompensierte Leberzirrhose, Sehstörungen, usw.), Schwere } \\
\text { neurologisch / psychiatrischen Erkrankungen, Drogen- und } \\
\text { Medikamentenabhängigkeit, Mangelnde deutsche Sprachkenntnisse, } \\
\text { Medikamenteneinnahme (v. a. zur Linderung von Entzugssymptomatik, } \\
\text { Benzodiazepine), keine Laborwerte oder älter als } 7 \text { Tage, Positiver } \\
\text { Alkoholtest (Alkohol in der Atemluft), BMI } \geq 40 \text {, Patienten, } \\
\text { die sich einer ärztlichen Untersuchung nicht unterzogen haben, } \\
\text { Probanden, die die Einverständniserklärung nicht unterschreiben. }\end{array}$ \\
\hline Kontrollgruppe & $\begin{array}{l}\text { Alter zwischen } 20 \text { und } \\
65 \text { Jahren, } \\
\text { Einverständnis zur } \\
\text { Teilnahme an der } \\
\text { Studie. }\end{array}$ & $\begin{array}{l}\text { Alkoholabhängigkeit oder eine andere Abhängigkeitserkrankung, } \\
\text { Einnahme von Beruhigungsmitteln, chronische somatische, neurologische } \\
\text { und/oder psychische Erkrankungen, positiver Alkoholtest (Alkohol in der } \\
\text { Atemluft), Einverständniserklärung nicht unterschrieben. }\end{array}$ \\
\hline
\end{tabular}

Kasten 1. Ein- und Ausschlusskriterien (Aus Tolic \& Soyka, 2018)

\subsection{Erfassungen der Stressreaktionen}

Cortisol im Speichel

Bei allen Probanden wurden Cortisolproben im Speichel entnommen. Zu den vorher festgelegten Zeitpunkten wurden insgesamt sechs Proben pro Person durchgeführt. Die Proben wurden zu folgenden Zeitpunkten entnommen: Bei der Ankunft als „Basalwert“ 10 min. vor Stressinduktion (T0), eine Minute vor Beginn der Stressinduktion (T1), eine Minute nach Stressinduktion (T2), fünf Minuten (T3), 30 Minuten (T4) und 90 Minuten nach Stressinduktion (T5). Unmittelbar nach der Durchführung der Stressinduktion, also bei T2, erhielten alle Probanden eine 
Ruhepause von fünf Minuten. In dieser Phase wurden jedem einzelnen Probanden auf einem PC-Bildschirm Ruhebilder gezeigt (Wald, Strand, Berge). Unmittelbar nach der Entnahme wurden alle Laborproben auf $-30{ }^{\circ} \mathrm{C}$ in einem Gefrierschrank bis zur laborchemischen Auswertung eingefroren.

Biologische Signale: Puls und elektrodermale Aktivität (EDA)

Die Erfassung von Puls und EDA erfolgte mit Hilfe von „Biosignalerfassungssystem Modul Multi 2000x-pert@“ von der Firma Psy Expert aus Mannheim. Beide Biosignale wurden von der Ankunft bis zur Beendigung der Untersuchung erfasst. Weitgehend angelehnt an die Durchführungsbedingungen des TSST wurden für die Erfassung fünf Messzeitpunkte festgelegt, und zwar bei TSST-Vorbereitung (T1), TSST-Rede (T2), TSST-Kopfrechnen (T3), in der Ruhephase (T4) und am Ende der Untersuchung (T5). Die Erhebungsinstrumente für die Diagnostik und Psychopathologie sind im Kapitel 4 dieser Dissertation vorgestellt.

\section{Ergebnisse}

Alle erhobenen Daten wurden mittels der Statistik- und Analyse-Software SPSS ausgewertet. Es wurden unterschiedliche varianzanalytische Auswertungen mit ANOVA einschließlich Messwiederholungen und Post-hoc-Tests nach Scheffé durchgeführt.

\subsection{Suchtspezifische Merkmale}

Hinsichtlich suchtspezifischer Merkmale ergaben sich zwischen den beiden Schweregradgruppen signifikante Unterschiede (s. Tabelle 1). Schwerkranke Patienten erlebten stärkeren Suchtdruck und wiesen eine längere Trinkdauer auf. Ebenso nahmen sie mehr stationäre Entzugs- und Entwöhnungsbehandlungen auf als leicht-mittelschwerkranke. Ein interessantes Ergebnis ergab sich im Hinblick auf die 
familiäre Vorgeschichte des Alkoholismus. Während 79,3\% der schwerkranken Alkoholabhängigen eine positive familiäre Vorgeschichte aufweisen, waren es bei den leicht-mittelschwerkranken nur 54,2\% $(p=.00)$. Unter den Schwerkranken befanden sich mehrere Personen mit einem richtig schweren Krankheitsverlauf insbesondere im Hinblick auf Trinkdauer, Suchtdruck und die Anzahl stationärer Entzugsbehandlungen.

\begin{tabular}{|c|c|c|c|}
\hline Merkmal & $\begin{array}{l}\text { Gruppe leicht-mittelschwer } \\
(\mathrm{N}=35)\end{array}$ & $\begin{array}{l}\text { Gruppe schwer } \\
(\mathrm{N}=29)\end{array}$ & Statistik \\
\hline Trinkdauer (Jahre) & $M=19,42( \pm 1,23)$ & $M=20,77( \pm 2,69)$ & $F(1,62)=13.33, p=.00$ \\
\hline Trinkmenge (Gramm/Tag) & $M=165,6( \pm 49,23)$ & $M=187,4( \pm 53,86)$ & $F(1,62)=2.859, p=.09$ \\
\hline Amb. Behandlungen & $M=1( \pm 1)$ & $M=1( \pm 1)$ & $F(1,62)=1.621, p=.20$ \\
\hline Stat. Entzugsbehandlung & $M=4( \pm 3)$ & $M=11( \pm 8)$ & $F(1,62)=15.94, p=.00$ \\
\hline Stat. Entwöhnungsbehandlung & $M=2( \pm 1)$ & $M=4( \pm 2)$ & $F(1,62)=7.049, p=.01$ \\
\hline Suchtberatung & $M=2( \pm 2)$ & $M=3( \pm 3)$ & $F(1,62)=3.284, p=.07$ \\
\hline Selbsthilfegruppe & $M=2( \pm 4)$ & $M=4( \pm 8)$ & $F(1,62)=1.535, p=.22$ \\
\hline Suchtdruck (MaCs) & $M=18,80( \pm 6,14)$ & $M=24,37( \pm 4,24)$ & $F(1,62)=17.11, p=.00$ \\
\hline Rauchen (Zigaretten/Tag) & $M=11,42( \pm 5,8)$ & $M=14,65( \pm 3,91)$ & $F(1,62)=1.053 . p=.30$ \\
\hline
\end{tabular}

*Mittelwertdifferenz ist auf der Stufe 0.05 signifikant. **Mittelwertdifferenz ist auf der Stufe 0.01 signifikant.

Tab. 1. Suchtspezifische Merkmale der Schweregradgruppen (aus Tolic \& Soyka, 2018)

7.2 Psychopathologie (Angst, Depression, einschneidende Lebensereignisse, Persönlichkeitsstörungen)

Betrachtet man die Ergebnisse der psychopathologischen Variablen so lässt sich feststellen, dass es lediglich zwischen Alkoholabhängigen und gesunden Probanden einen signifikanten Unterschied im Bereich Ängstlichkeit (STAl) gab ( $p=.00)$, zwischen den beiden Schweregradgruppen hingegen nicht $(p=.13)$. Im Bezug auf depressive Symptome weisen sowohl Alkoholabhängige als auch gesunde Probanden normale Werte im Becks-Depressions-Inventar (BDI) auf (s. Diagramm 1 im Anhang). Im Gegensatz dazu erlebten Alkoholabhängige mehr einschneidende Lebensereignisse (ILE) als gesunde Probanden $(p=.00)$. Ebenso zeigte die 
Subgruppe der Schwerkranken eine stärkere Belastung als leicht-mittelschwer $(p=.01)$. Hinsichtlich des Auftretens von Persönlichkeitsstörungen konnten keine wesentlichen Unterschiede zwischen den beiden Substichproben Alkoholabhängiger (schwer und leicht-mittelschwer) gefunden werden. In der Teilstichprobe der Schwerkranken konnte bei vier Patienten eine emotional instabile Persönlichkeitsstörung vom Borderline-Typus und bei zwei Patienten eine ängstlich vermeidende Persönlichkeitsstörung diagnostiziert werden. In der Teilstichprobe der Leicht-mittelschwer konnte bei zwei Patienten eine Borderline- und bei weiteren drei Patienten jeweils eine dependente, ängstlich vermeidende und eine narzisstische Persönlichkeitsstörung diagnostiziert werden. In der Stichprobe der gesunden Probanden konnten keine Persönlichkeitsstörungen diagnostiziert werden.

\subsection{Biologische Variablen}

\subsubsection{Hämatologie}

Im Bereich der Hämatologie (Gamma-Gt, GOT, GPT, MCV) haben sich signifikante Unterschiede in den Subgruppen Alkoholabhängiger ergeben. Zunächst der Vergleich im Hinblick auf Schweregrad. Die Schwerkranken mit einer Abstinenz von sieben Tagen (A7-schwer) zeigten signifikant höhere Leberenzym- und MCV-Werte als Patienten der Subgruppe A7-leicht-mittelschwer (z. B. Gamma-GT p=.00, GOT $\mathrm{p}=.03$ ). Zwischen A70-schwer und A70-leicht-mittelschwer ergaben sich keine signifikanten Unterschiede. Im Bezug auf Abstinenzdauer zeigten die Patienten der A7 Gruppe wie erwartet signifikant höhere Werte als die Gruppe A70 $(p=.01)$.

\subsubsection{Cortisol}

Alle Teilnehmer haben auf TSST als Stressinduktionsmethode mit signifikanter Erhöhung der Cortisolwerte reagiert. Zunächst wurden die Ergebnisse zwischen Alkoholabhängigen und gesunden Probanden verglichen. In beiden Stichproben 
weisen Probanden zu Beginn der Untersuchung normale Cortisolwerte im Speichel auf

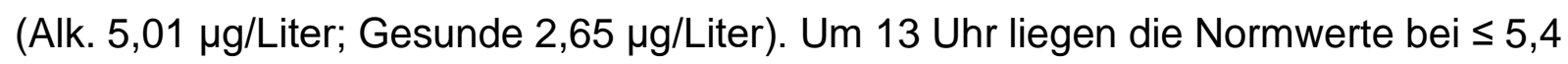

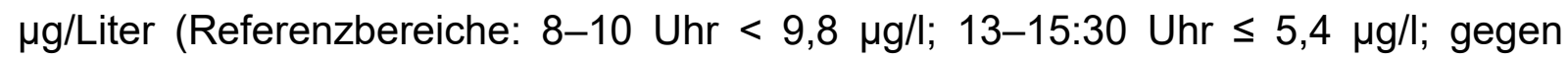
Mitternacht $<2 \mu \mathrm{g} / \mathrm{l})$. Bei allen sechs Messzeitpunkten bestand ein signifikanter Unterschied zwischen beiden Stichproben (T0 $p=.00 ;$ T1 $p=.00 ;$ T2 $p=.02 ;$ T3 $p=.00$; T4 $\mathrm{p}=.00 ; \mathrm{T} 5 \mathrm{p}=.00)$. Diese Ergebnisse machen deutlich, dass Aufgrund der unterschiedlichen Abstinenzdauer und der Krankheitsschwere bei Alkoholabhängigen eine differenziertere Auswertung nach Teilstichproben notwendig ist. Im zweiten Schritt wurden die Teilstichproben nach Schweregrad untereinander (schwer und leicht-mittelschwer) und mit gesunden Probanden verglichen. Schwerkranke Patienten zeigten zu Beginn der Untersuchung (T0) leicht erhöhte Cortisolwerte $(5,8 \mu \mathrm{g} / \mathrm{l})$ und diese sind signifikant höher als in den Teilstichproben Leicht-mittelschwer $(4,45 \mu \mathrm{g} / \mathrm{l}$, $p=.03)$ und Gesunde (2,65 $\mu g / l, p=.00)$. Beim Zeitpunkt T2, also nach der Stressinduktion, reagierten gesunde Probanden mit einer stärkeren Cortisolstressantwort als Patienten in den beiden anderen Teilstichproben. Zu diesem Messzeitpunkt bestehen keine signifikanten Unterschiede zwischen gesunden Probanden und den Schwerkranken $(p=.10)$ sowie zwischen gesunden und den Patienten der Teilstichprobe leicht-mittelschwer $(p=.69)$. Genau an dieser Stelle begannen schwerkranke Patienten eine Verzögerung in der Stressreaktion zu zeigen (s Diagramm 2). In der Ruhephase, also fünf Minuten nach Beendigung der Stressinduktion, zeigten sich die Unterschiede zwischen den Teilstichproben. Bei den gesunden Probanden sanken die Werte um mehr als zwei Mikrogramm pro Liter. Während dessen stiegen sie bei beiden Patientenstichproben noch weiter an (Mehrfachvergleiche, s. Tab. 2 im Anhang). Eine halbe Stunde nach Beendigung der Stressinduktion (T4) werden auch bei Alkoholabhängigen die Cortisolwerte rückläufig, jedoch langsamer als bei gesunden Probanden. Am Ende der Untersuchung (T5) 
sinken die Cortisolwerte sowohl bei gesunden Probanden $(4,06 \mu \mathrm{g} / \mathrm{l})$ als auch in der Patientengruppe leicht-mittelschwer $(5,42 \mu \mathrm{g} / \mathrm{l})$ fast auf das Ausgangsniveau zurück. Mit einem Ergebnis von 7,83 $\mu \mathrm{g} / \mathrm{l}$ liegen die Werte bei schwerkranken Patienten hingegen $2 \mu \mathrm{g} / \mathrm{l}$ höher als zu Beginn der Untersuchung und unterscheiden sich sehr signifikant von den anderen (Teil-)Stichproben ( $p=.00)$. Insgesamt lässt sich aber feststellen, dass sowohl Schwerkranke, mit einem Cortisolanstieg von 4,25 $\mu \mathrm{g} / \mathrm{l}$, als auch Leicht- mittelschwerkranke, mit einem Cortisolanstieg von 3,82 $\mu \mathrm{g} / \mathrm{l}$, eine abgeschwächte Stressreaktion im Vergleich zu gesunden Probanden $(6,71 \mu \mathrm{g} / \mathrm{l}$, $\mathrm{p}=.00)$ zeigen.

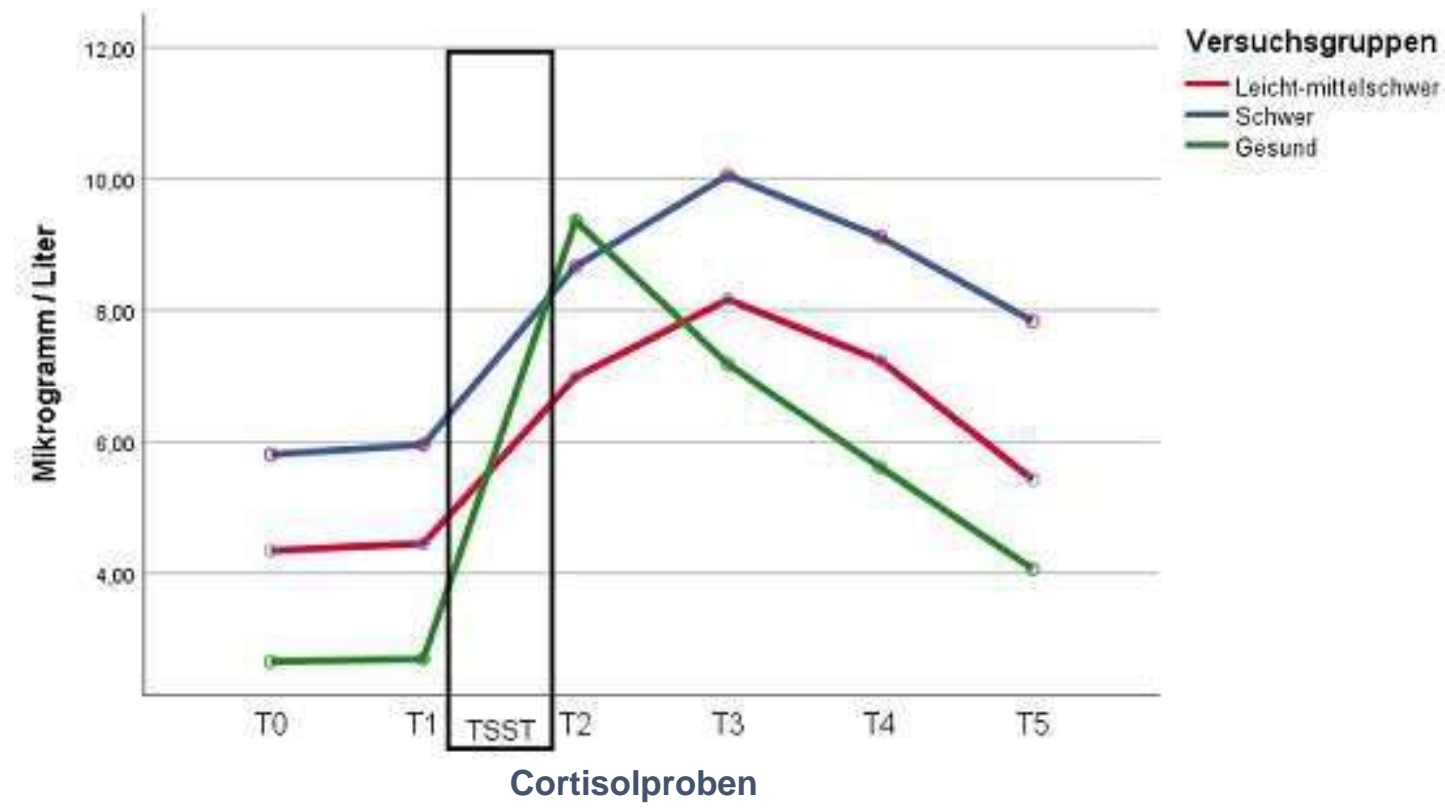

Diagramm 2. Cortisol: Verlaufskurven der Versuchsgruppen aufgeteilt nach Schweregrad (aus Tolic \& Soyka, 2018).

Bei dem dritten Schritt der Ergebnisauswertung erfolgte die Aufteilung der alkoholabhängigen Patienten sowohl nach Schweregrad als auch nach der Abstinenzdauer. Um eine genauere Interpretation der Ergebnisse zu gewährleisten, ist dieser noch differenziertere Auswertungsschritt erforderlich. 
-Aufteilung nach Schweregrad und Abstinenzdauer (A7-schwer, A7-leicht-mittelschwer, A70-schwer, A70-leicht-mittelschwer)

Bei einer gleichzeitigen Berücksichtigung der Abstinenzdauer und der Krankheitsschwere ergaben sich in der Auswertung nach ANOVA signifikante Unterschiede zwischen den Gruppen. Die Patienten der Teilstichprobe A7-schwer zeigten zu Beginn der Untersuchung erhöhte Cortisolwerte $(7,51 \mu \mathrm{g} / \mathrm{l})$. Mittels Posthoc-Auswertung nach Scheffé unterscheiden sie sich von der Teilstichprobe A7-leichtmittelschwer $(4,88 \mu \mathrm{g} / \mathrm{l})$ signifikant und von allen anderen Teilstichproben sehr signifikant $(p=.00)$. Beim Messzeitpunkt T1 ergaben sich hingegen keine signifikanten Unterschiede zwischen A70-schwer und A70-leicht-mittelschwer sowie zwischen diesen beiden Subgruppen und den gesunden Probanden. Insbesondere bei der letzten Speichelprobe (T5) zeigten Patienten der Teilstichprobe A7-schwer mit einem Ergebnis von 9,09 $\mu \mathrm{g} / \mathrm{l}$ deutlich höhere Cortisolwerte als A7-leicht-mittelschwer $(6,36 \mu g / l ; p=.00)$, A70-schwer $(6,76 \mu g / l ; p=.02)$ als auch A70-leicht-mittelschwer $(4,54 \mu \mathrm{g} / \mathrm{l})$ und gesunde Probanden (jeweils $p=.00)$. Die Teilstichprobe A70-schwer zeigte signifikant höhere Werte im Vergleich zu A70-leicht-mittelschwer $(p=.04)$ und mit Gesunden $(p=.00)$. Bei allen anderen Messzeitpunkten ergaben sich signifikant höhere Cortisolwerte bei schwerkranken Patienten, also bei A7-schwer und bei A70schwer, unabhängig von der Abstinenzdauer (s. Tabelle 3 im Anhang).

\subsubsection{Elektrodermale Aktivität (EDA)}

Die Elektrodermale Aktivität (EDA) aktiviert zuverlässig das sympathoadrenomedulläre System. Sie gilt als sensible physiologische Reaktionsgröße für die Neuheit eines Reizes. Zu Beginn der Untersuchung zeigten Alkoholabhängige höhere EDA-Basiswerte (Messzeitpunkt 1) gemessen in Mikrosiemens ( $\mu S$ ) als gesunde Probanden ( $p=.04)$. Normwerte liegen zwischen 1 und $15 \mu \mathrm{S}$. Für diese Studie ist die 
Höhe des Leitwertes von sekundärer Bedeutung. Seine Veränderung in Abhängigkeit von der Stressinduktion ist viel mehr von Relevanz. Bei der freien Rede (2) und beim Kopfrechnen (3), also während der Stressinduktion, stiegen sowohl bei den Alkoholkranken als auch bei den gesunden Probanden die Werte rasant an. Sie unterscheiden sich zu diesen beiden Zeitpunkten nicht $(p=.56)$. Erst am Ende der Untersuchung fiel das Ergebnis signifikant aus (M-alk = 3,74 $\mu \mathrm{S} ; \mathrm{M}$-ges = 2,90 $\mu \mathrm{S}$; $F(1,92)=9,73 ; p=.00)$. Nach Schweregrad aufgeteilt unterscheiden sich Schwerkranke $(M-s c h w e r=3,25)$ von den leicht-mittelschweren $(M-L e i c h t-m .=2,75)$ in ihren Basiswerten (1) nicht signifikant $(p=.16)$. Von den Gesunden (M-ges $=2,50)$ jedoch schon $(p=.02)$. Weitere Ergebnisse nach dieser Aufteilung befinden sich weiter unten (Tabelle 4a) und der dazugehörige Varianzhomogenitätstest mit der Varianzanalyse (ANOVA) im Anhang (s. Tabelle 4b im Anhang). Aufgeteilt nach beiden Kriteriumsvariablen, Schweregrad und Abstinenzdauer, ergaben sich zwischen den Teilstichproben im Bereich der Basiswerte (1) signifikante Unterschiede $(F(4,89)=4,26 ; p=.00)$. Bei Mehrfachvergleichen weisen schwerkranke Patienten mit einer Abstinenzdauer von 70 Tagen (A70-schwer) signifikant höhere EDABasiswerte als A7-leicht-mittelschwer $(p=.02)$ und Gesunde $(p=.04)$ auf. Alle anderen Teilstichproben unterscheiden sich nicht voneinander. In der freien Rede zeigten Patienten der Gruppe A70-schwer signifikant höhere EDA-Werte als A7-schwer $(p=.01)$ sowie A7-leicht-m. $(p=.02)$. Zwischen allen anderen Gruppen konnten keine Unterschiede festgestellt werden. Bei der Kopfrechenaufgabe zeigten Patienten der Teilstichproben A70-schwer und A70-leicht-m. höhere Werte als A7-schwer und A7leicht-mittelschwer (A70-schwer u. A7-schwer $p=.00$; A70-schwer u. A7-leicht-m. $\mathrm{p}=.00 ;$ A70-leicht-m. und A7-leicht-m. $\mathrm{p}=.00$ ). Aber auch gesunde Probanden zeigten in dieser Experimentalbedingung höhere Werte als A7-leicht-m. $(p=.01)$. Alle anderen Vergleiche waren nicht signifikant. In Ruhe zeigen sowohl A7-schwer als auch A70- 
schwer signifikant höhere EDA-Werte als Gesunde $(p=.01$, und $p=.03)$. Alle anderen Teilstichproben unterscheiden sich nicht voneinander. Am Ende der Untersuchung haben schwerkranke Patienten mit einer Abstinenzdauer von sieben Tagen (A7) signifikant höhere Werte als A70-leicht-m. $(p=.01)$ und Gesunde $(p=.00)$. Auch Patienten der Teilstichprobe A70-schwer zeigten signifikant höhere EDA-Werte als A70-leicht-m. $(p=.04)$ und Gesunde $(p=.00)$. Die EDA-Werte gesunder Probanden unterscheiden sich von Werten der Patienten A7-leicht-m. $(p=.66)$ und A70-leicht-m. $(p=.99)$ am Ende der Untersuchung nicht. Insgesamt zeigen nur schwerkranke Patienten, unabhängig von ihrer Abstinenzdauer, eine stärkere Stressreaktion und unterscheiden sich signifikant von leicht-mittelschwerkranken Patienten und gesunden Probanden.

\begin{tabular}{|c|c|c|c|c|c|c|c|}
\hline EDA-Messzeitpunkte & Teilstichproben & $\bar{N}$ & $\mathbf{M}$ & SD & Df & $F$ & Sig. \\
\hline 1 = 1 min. vor TSST & $\begin{array}{l}\text { Leicht-mittelschwer } \\
\text { Schwer } \\
\text { Gesund }\end{array}$ & $\begin{array}{l}35 \\
29 \\
30\end{array}$ & $\begin{array}{l}2,75 \\
3,25 \\
2,50\end{array}$ & $\begin{array}{l}0,98 \\
1,07 \\
1,02\end{array}$ & 2,91 & 4.013 & $.02^{*}$ \\
\hline 2 = TSST - freie Rede & $\begin{array}{l}\text { Leicht-mittelschwer } \\
\text { Schwer } \\
\text { Gesund }\end{array}$ & $\begin{array}{l}35 \\
29 \\
30\end{array}$ & $\begin{array}{l}8,24 \\
8,58 \\
8,73\end{array}$ & $\begin{array}{l}1,91 \\
2,52 \\
3,42\end{array}$ & 2,91 & 0.296 & .74 \\
\hline 3 = TSST - Kopfrechnen & $\begin{array}{l}\text { Leicht-mittelschwer } \\
\text { Schwer } \\
\text { Gesund }\end{array}$ & $\begin{array}{l}35 \\
29 \\
30\end{array}$ & $\begin{array}{l}6,96 \\
8,09 \\
7,61\end{array}$ & $\begin{array}{l}2,73 \\
2,42 \\
2,88\end{array}$ & 2,91 & 1.437 & .24 \\
\hline $\begin{array}{l}4 \text { = Ruhe } \\
\text { (5 min. nach TSST) }\end{array}$ & $\begin{array}{l}\text { Leicht-mittelschwer } \\
\text { Schwer } \\
\text { Gesund }\end{array}$ & $\begin{array}{l}35 \\
29 \\
30\end{array}$ & $\begin{array}{l}4,07 \\
4,73 \\
3,25\end{array}$ & $\begin{array}{l}1,29 \\
1,46 \\
1,20\end{array}$ & 2,91 & 9.362 & $.00^{* *}$ \\
\hline $\begin{array}{l}5 \text { = Ende } \\
\text { (90 min. nach TSST) }\end{array}$ & $\begin{array}{l}\text { Leicht-mittelschwer } \\
\text { Schwer } \\
\text { Gesund }\end{array}$ & $\begin{array}{l}35 \\
29 \\
30\end{array}$ & $\begin{array}{l}3,22 \\
4,36 \\
2,90\end{array}$ & $\begin{array}{l}0,99 \\
1,33 \\
1,05\end{array}$ & 2,91 & 13.761 & $.00^{* \star}$ \\
\hline
\end{tabular}

${ }^{*}$ Mittelwertdifferenz ist auf der Stufe 0.05 signifikant. ${ }^{* *}$ Mittelwertdifferenz ist auf der Stufe 0.01 signifikant.

Tab. 4a. EDA: Teilstichproben aufgeteilt nach Schweregrad (aus Tolic \& Soyka, 2018).

\subsubsection{Puls}

Die Pulswerte zeigten sich zu Beginn der Untersuchung (1) sowohl bei Alkoholabhängigen als auch bei gesunden Probanden im Normbereich. Die Normwerte bei Erwachsenen liegen zwischen 60 und 80 Schlägen pro Minute. 
Ähnlich wie bei der EDA stieg der Pulswert während der Stressinduktion bei beiden Stichproben deutlich an. Am Ende der Untersuchung (5) ergab sich ein signifikanter Unterschied zwischen den Gruppen (Alk. $M=82$, Gesunde $M=77 ; F(1,92)=16,50$; $\mathrm{p}=.00$ ). Betrachtet man die Teilstichproben aufgeteilt nach Schweregrad, so ergaben sich für die jeweiligen Untersuchungsparadigmen unterschiedliche Resultate (s. Tabelle 5 im Anhang). Während es in der Vorbereitung auf TSST (1) signifikante Unterschiede zwischen den Gruppen gab ( $F 2,91=4.656, p=.01)$, sind diese weder bei der Rede $(F(2,91)=2.630, p=.07)$ noch bei der Kopfrechenaufgabe nachweisbar $(\mathrm{F}(2,91)=3.079, \mathrm{p}=.051)$. Erst bei der Ruhebedingung $(\mathrm{F}(2,91)=11.857, \mathrm{p}=.00)$ und am Ende (5) der Untersuchung werden die Unterschiede wieder sehr signifikant $(F(2,91)=12.040, p=.00)$. Schwerkranke Patienten weisen höheren Ruhepuls auf. Dieser wird in den Mehrfachvergleichen nach Scheffé deutlich. In der Vorbereitung unterscheiden sich die Patienten der Stichprobe Schwer von gesunden Probanden signifikant $(p=.01)$ nicht aber von der Gruppe Leicht-mittelschwer $(p=.10)$. Ebenso ergaben sich keine Unterschiede zwischen Leicht-mittelschwer und Gesund $(p=.63)$. Während der Rede stieg der durchschnittliche Pulswert in allen (Teil-) Stichproben an. Weder bei dieser Bedingung noch bei der Kopfrechenaufgabe konnten signifikante Unterschiede zwischen den Gruppen festgestellt werden. Erst in Ruhe unterscheiden sich die Werte schwerkranker Patienten von denen gesunder Probanden signifikant $(p=.00)$ und ebenso Leicht-mittelschwer von Gesunden $(p=.04)$. Schwer und Leicht-mittelschwer unterscheiden sich dagegen nicht voneinander $(p=.05)$. Am Ende der Untersuchung ergaben sich bei schwerkranken Patienten signifikant höhere Pulswerte (84/Minute) im Vergleich zu Leicht-m. (80; $p$ $=.04)$ als auch zu gesunden Probanden $(77 ; p=.00)$. Ähnliche Ergebnisse ergaben sich bei der Aufteilung nach Abstinenzdauer und Krankheitsschwere. Insgesamt zeigten insbesondere die Patienten der Teilstichprobe A70-schwer signifikant höhere 
Pulswerte als beispielsweise die Patienten der Teilstichprobe A70-Leicht-m. $(p=.04)$ und Gesunde $(p=.00)$.

\subsection{Stressverarbeitung}

Die Ergebnisse der Stressverarbeitung wurden mittels Stressverarbeitungsfragebogen (SVF 120), Alltagsbelastungsfragebogen (ABFB) und Trierer Inventar zum chronischen Stress (TICS) gewonnen.

\subsubsection{Stressverarbeitungsfragebogen (SVF 120)}

Im Stressverarbeitungsfragebogen zeigten Alkoholabhängige in mehreren Bereichen sowohl signifikant höhere als auch signifikant niedrigere Werte als Gesunde. Signifikant höhere Werte ergaben sich in folgenden Subskalen: Bagatellisierung $(p=.00)$, Schuldabwehr $(p=.01)$, Soziales Unterstützungsbedürfnis $(p=.00)$, Vermeidung $(p=.02)$, Flucht $(p=.01)$, Soziale Abkapselung $(p=.01)$, Gedankliche Weiterbeschäftigung $(p=.00)$, Resignation $(p=.00)$, Selbstbemitleidung $(p=.04)$ und Selbstbeschuldigung $(p=.02)$. Signifikant niedrigere Werte in folgenden Bereichen: Ersatzbefriedigung $(p=.03)$, Selbstbestätigung $(p=.00)$, Entspannung $(p=.00)$, Reaktionskontrolle $(p=.03)$ und Positive Selbstinstruktionen $(p=.00)$. Keine Unterschiede konnten dagegen in den Bereichen Herunterspielen ( $p=.053)$, Ablenkung $(p=.13)$, Situationskontrolle $(p=.17)$ und Aggression $(p=.29)$ gefunden werden. Insgesamt wählten Alkoholabhängige überdurchschnittlich häufiger negative Stressverarbeitungsstrategien als gesunde Probanden $\quad(\mathrm{M}$-alk $=62,46, \quad \mathrm{M}$ ges $=49,93, \quad F(1,92)=73.202, \quad p=.00)$. Gleichzeitig wählten sie die positiven Strategien signifikant weniger häufig als Gesunde (M-alk $=44,85$, M-ges $=54,86$, $F(1,92)=58.861, p=.00)$. Im Hinblick auf Krankheitsschwere wählten Gesunde signifikant häufiger positive Stressverarbeitungsstrategien als schwerkranke Patienten $(M-g e s=54,86, p=.00)$ und Leicht-mittelschwer $(p=.00)$. Bei den negativen 
Strategien wählten Schwerkranke diese überdurchschnittlich häufiger als Leichtmittelschwer (M-schwer $=64,93, M$ - leicht-m. $=60,42, p=.02)$ und Gesunde (Mges $=49,93, p=.00)$. Eine Tabelle, in der die Mehrfachvergleiche aller 20 Subskalen des SVF abgebildet werden, ist zu groß. Deshalb werden die Ergebnisse der Positivund Negativ-Strategien des SVF zusammengefasst in der Tabelle 6 (s. Anhang) dargestellt.

\subsubsection{Alltagsbelastungsfragebogen (ABF)}

Alkoholabhängige und Gesunde zeigten im Alltagsbelastungsfragebogen keine signifikanten Unterschiede $(\mathrm{M}$-alk $=13,30, \mathrm{M}$-ges $=10,85, \mathrm{~F}(1,92)=.837 ; \mathrm{p}=.36)$. Selbst unter einer differenzierteren Betrachtung nach Schweregrad haben sich keine signifikanten Unterschiede zwischen den Teilstichproben ergeben $(\mathrm{M}$-schwer $=$ 15,09, M-leicht-m. $=11,82, \mathrm{M}$-ges $=10,25, F(2,91)=.996, p=.37)$.

\subsubsection{Trierer Inventar zum chronischen Stress (TICS)}

In der Screening-Skala des TICS weisen Alkoholabhängige in dieser Studie überdurchschnittlich hohe chronische Stressbelastung im Vergleich zu gesunden Probanden auf (M-Alk $=64,59$, M-ges $=55,80, F(1,92)=112,45 ; p=.00)$. Der Normwert liegt bei $50 \pm 6,6$ Punkte. In den Subskalen Soziale Überlastung ( $p=.03$ ), Arbeitsüberlastung $(p=.01)$, Mangel an sozialer Anerkennung $(p=.00)$, Soziale Spannungen $(p=.00)$, Soziale Isolation $(p=.00)$ und chronische Besorgnis $(p=.00)$ zeigten Alkoholabhängige signifikant höhere Werte als Gesunde. In den Subskalen Erfolgsdruck ( $\mathrm{p}=.88$ ), Unzufriedenheit mit der Arbeit $(\mathrm{p}=.05)$ und Überforderung bei der Arbeit $(p=.29)$ unterscheiden sich die beiden Stichproben hingegen nicht signifikant voneinander. Teilt man die Stichprobe Alkoholabhängiger nach Schweregrad auf (Tabelle 6 im Anhang), so zeigen diese signifikant höhere Werte chronischer Stressbelastung als gesunde Probanden $(p=.00)$. 


\subsection{Korrelationsanalyse}

Mittels zweiseitiger Prüfung korreliert der Schweregrad der Alkoholabhängigkeit nach Pearson in dieser Studie signifikant mit mehreren Variablen, unter anderem mit Gamma-GT, MCV, Cortisolwerten, EDA, Trinkdauer, Anzahl an Behandlungen und Suchtdruck, Stressbewältigungsstrategien (positiv und negativ) sowie akuten und chronischen Stressbelastungen (s. Tabelle 8 im Anhang). Auch mittels Multivariater Varianzanalyse ergaben sich nach Bonferroni-Korrektur signifikante Ergebnisse für die Variable Schweregrad. Der Schweregrad der Abhängigkeit korreliert mit folgenden Variablen hingegen nicht: Trinkmenge $(p=.09)$, Rauchen $(p=.30)$,SVFHerunterspielen $(p=.85)$, SVF-Schuldabwehr $(p=.96)$, SVF-Ablenkung $(p=.41)$, SVFErsatzbefriedigung $(p=.30)$ und Alltagsbelastung $(p=.28)$

Für die Variable Abstinenzdauer ergaben sich ähnliche Ergebnisse (Tabelle $8 \mathrm{im}$ Anhang). Sie korreliert u.a. ebenfalls mit den Leberenzymen (Gamma-GT, GOT, GPT), mit MCV, mit dem Ausmaß an alläglichem Stress, sozialen Spannungen, Ausmaß an Angst und Depression, Cortisolwerten, EDA sowie Stressbewältigungsstrategien. Schließlich wurde innerhalb der Stichprobe der Alkoholabhängigen eine lineare Regressionsanalyse mit allen Variablen als Prädiktoren und dem Cortisol (Cortisolwert-T5) als Kriterium für das Ausmaß der Stressreaktion durchgeführt (s. Tabelle 9 im Anhang). Die Prädiktoren trugen gemeinsam 75,4\% zur Varianzaufklärung bei (korrigiertes $\mathrm{R}^{2}=.754 ; \mathrm{F}(17,46)=5.195, \mathrm{p}=.00$ ). Die beiden wichtigsten Prädiktoren, der Schweregrad und die Abstinenzdauer, klären alleine fast $35 \%$ der Varianz auf (korrigiertes $R^{2}=.345 ; F(2,61)=17.558, p=.00$ ). Die Prädiktoren der beiden anderen Kriteriumvariablen Puls und EDA trugen geringer, aber immer noch sehr signifikant zur Varianzaufklärung bei. Beim Puls klärten die Prädiktoren $15,2 \%$ der Varianz auf (korrigiertes $R^{2}=.152 ; F(2,61)=6.643, p=.00$ ) und bei der EDA 28,7 \% (korrigiertes $\left.\mathrm{R}^{2}=.287 ; \mathrm{F}(2,61)=13.699, \mathrm{p}=.00\right)$. 


\section{Diskussion}

In der durchgeführten Studie wurde geprüft, ob die Schwere der Alkoholabhängigkeit bei der Stressreagibilität und Stressverarbeitung eine Rolle spielt. Außerdem wurde geprüft, ob sich schwerkranke Alkoholabhängige von leicht-mittelschwerkranken und gesunden Probanden im Hinblick auf Stressreagibilität und Stressverarbeitung unterscheiden. Die Ergebnisse zeigen, dass sowohl die Krankheitsschwere als auch die Abstinenzdauer einen Einfluss auf die Stressreagibilität und Stressverarbeitung haben. Zudem unterscheiden sich schwerkranke Alkoholabhängige in ihren Ergebnissen von den leicht-mittelschwerkranken und gesunden Probanden. Außerdem zeigen schwerkranke Patienten in dieser Studie eine prolongierte Stressreaktion. Normalerweise erreicht die Cortisolkonzentration im Speichel und im Blut ihre Höhepunkte 15-30 Minuten nach der Stressinduktion. Etwa 60-90 Minuten danach fallen sie wieder auf ihr Ausgangsniveau zurück (de Kloet et al., 2005; Kudielka et al., 2007). Bei schwerkranken Patienten sind weder Cortisol- noch EDAoder Pulswerte in diesem Zeitraum auf das Ausgangsniveau zurückgegangen. Zu Beginn der Untersuchung weisen lediglich schwerkranke Alkoholabhängige mit einer Abstinenzdauer von sieben Tagen (A7) erhöhte Cortisolwerte auf. Alle anderen Patienten und gesunde Probanden hingegen nicht. Ähnliche Werte fand man auch bei den Variablen EDA und Puls. Erfreulich war die Tatsache, dass alle Untersuchungsteilnehmer auf TSST als Stressinduktionsmethode reagiert haben. So stieg der Cortisolwert bei den gesunden Probanden durch die Stressinduktion sehr stark an. Bei den schwerkranken, aber auch bei den leicht-mittelschwerkranken Patienten mit einer Abstinenzdauer von sieben Tagen (A7-schwer und A7-leichtmittelschwer) ergab sich kein starker Cortisolanstieg wie bei Gesunden. Dieses Ergebnis ist mit anderen Studien vergleichbar, wonach Alkoholabhängige in der frühen Abstinenzphase eine abgeschwächte HHNA-Reaktion aufweisen (Fox et al., 2007; 
Sinha et al., 2009; Sinha, 2011; Junghanns et al., 2003). Aber diese Studien vergleichen nicht, ob und wie sich diese Reaktion nach einer angemessenen Abstinenzdauer, z. B. 70 Tage, verändert. In dieser Studie zeigte sich, dass neben der Abstinenzdauer auch die Krankheitsschwere bei der Stressreagibilität eine Rolle spielt. Betrachtet man die Ergebnisse der Krankheitsschwere (A7-schwer und A70schwer), so kann man feststellen, dass diese Patienten unabhängig von der Abstinenzdauer (7 oder 70 Tage) eine verlängerte Stressreaktion zeigten. Der Beginn der neuroendokrinen Stressreaktion (Cortisol) bei Schwerkranken ist verzögert und dauert länger als bei leicht-mittelschwerkranken Patienten und Gesunden. Sie normalisiert sich bei Schwerkranken nach 90 Minuten nicht. Dieser Befund ist neu und könnte für eine effektive Rückfallprophylaxe bei Alkoholabhängigen in der frühen Abstinenzphase von Relevanz sein. In dieser Phase sind die Patienten verschiedensten Trinkreizen ausgesetzt und erleben vermehrt Stress, Ängste und Unsicherheiten, weshalb die Gefahr für einen Alkoholrückfall besonders groß ist (Beispielübersicht bei Sinha, 2012, Blaine et al., 2016, Becker, 2008). Neu an dieser Studie ist auch die gleichzeitige Berücksichtigung von mehreren, teilweise essentiell wichtigen Variablen wie der Abstinenzdauer, der Krankheitsschwere, als auch des akuten und chronischen Stresses, suchtspezifischer Variablen (Leberenzyme, Suchtdruck), der akuten und chronischen Stressverarbeitung, psychopathologischen Variablen (BDI, STAI, SKID-II), alltäglichen Belastungen (ABF) und lebensverändernden Ereignissen (ILE). Die meisten Studien berücksichtigten nur die Abstinenzdauer oder die Krankheitsschwere, dabei die letztere häufig nur in Form von Trinkmengen. Die Abstinenzdauer wurde nur in ein Paar Studien mit sehr unterschiedlichem Patientenkollektiv (abhängig, nicht abhängig, „Sozialtrinker“) berücksichtigt, die beispielsweise zwischen zwei und sechs Wochen bis zu zwei Jahren sowie zwischen dreieinhalb und fünf Jahren abstinent waren (Junghanns et 
al., 2003; Starke et al., 2013; Munro et al., 2005). Außerdem unterscheiden sich die Stressinduktionstechniken sehr stark und wurden beispielsweise durch imaginative Techniken durchgeführt. Wie schon erwähnt waren bei den meisten Studien die Stichproben mit alkoholabhängigen und nichtalkoholabhängigen Personen recht heterogen und mit nur 18 alkoholabhängigen Patienten beispielsweise sehr klein (Munro et al., 2005; Fox et al., 2007). Das erschwert die Generalisierbarkeit der Ergebnisse. Hinzu kommt, dass zwischen akutem und chronischem Stress sowie alltäglichen Belastungen und lebensverändernden Ereignissen gleichzeitig in keiner Studie unterschieden wurde. Außerdem wurde in diesen Studien die Stressverarbeitung ebenfalls mit sehr unterschiedlichen Testverfahren durchgeführt und in manchen Studien (Aldridge-Gerry et al., 2011) mit der Auswahl von einem sehr speziellen Untersuchungskollektiv (Studenten mit unterschiedlicher ethnischer Zugehörigkeit). Die Krankheitsschwere wurde lediglich in einer Studie berücksichtigt, die an abstinent lebenden und rückfälligen Alkohol- und Opiatabhängigen durchgeführt wurde, in der die Forscher von verstärkter Nutzung maladaptiver Copingstrategien berichteten (Matto et al., 2009). Hierbei wurde aber nur die Stressverarbeitung berücksichtigt und nicht die Stressreagibilität auf akuten Stress. In einer anderen Studie wurde der Stressverarbeitungsfragebogen (SVF) eingesetzt bei rückfälligen Patienten mit einer Abstinenzdauer von einem Tag und noch einmal nach 40 Tagen (Walter et al., 2006). Die Stressreagibilität und die Krankheitsschwere wurden aber nicht berücksichtigt. Alkoholabhängige weisen in dieser Studie stärkere Flucht- und Vermeidungsreaktionen im Bereich der Stressverarbeitung auf. Auch dieses Ergebnis ist mit anderen Studien vergleichbar (Mattoo et al., 2009; AldridgeGerry et al., 2011; Walter et al., 2006). Besonders stark bei den schwerkranken Patienten waren die gedankliche Weiterbeschäftigung, Fluchtreaktion und sehr hohes soziales Unterstützungsbedürfnis als Stressverarbeitungsstrategien 
ausgeprägt. Möglicherweise "grübeln“ schwerkranke Patienten über belastende Inhalte länger. Die Konsequenz könnte sich vielleicht in der weniger erfolgreichen Bewältigung von sozialen Problemen niederschlagen. Dieses Ergebnis deckt sich mit dem Ergebnis in der Subskala chronische Besorgnis des TICS als chronische Stressbelastung. Im Hinblick auf allgemeine alltägliche Belastungen (ABF) unterscheiden sich Alkoholabhängige von den gesunden Probanden in dieser Studie nicht. Vielmehr ergaben sich im Bereich vom chronischen Stress starke Unterschiede zwischen Alkoholabhängigen und Gesunden. Insbesondere die schwerkranken Patienten zeigten sich schwer belastet und klagten häufiger über Mangel an sozialer Anerkennung, soziale Konflikte und Isolation als leicht-mittelschwere und Gesunde. Außerdem zeigten Schwerkranke längere Trinkdauer, stärkeren Suchtdruck, stärkere familiäre Vorbelastung, erlebten mehr einschneidende Lebensereignisse und nahmen mehr Behandlungen in Anspruch als leicht-mittelschwerkranke Patienten. Allerdings unterscheiden sich Schwerkranke von den beiden anderen (Teil-) Stichproben in dieser Studie nicht in allen Variablen. Dies gilt insbesondere für die Ergebnisse im ABFB und in Teilbereichen des SVF-120. Wie jede Studie hat auch diese ihre Einschränkungen. Hierbei sind insbesondere die moderaten Fallzahlen in den jeweiligen Stichproben zu erwähnen. Wenn man versucht die einzelnen Effekte so differenziert wie möglich herauszuarbeiten, mussten die Stichproben in Teilstichproben aufgeteilt werden, was die Fallzahlen weiter verringerte. Ebenfalls könnte man im Bereich der Schweregradbestimmung bei der Selbstbeurteilung durch den Patienten oder durch Interviewereffekte trotz größter Sorgfalt beim Vorgehen nicht ausschließen, dass sich Antworten verändern. Es sind Untersuchungen mit größeren Fallzahlen erforderlich, um die Ergebnisse zu bestätigen. 


\section{Fazit}

Alkoholkonsum ist ein weit verbreitetes Phänomen. Man kann sagen, dass der Alkohol im Rahmen der Menschheitsgeschichte fest verankert ist. Er ist eine legale Droge, deren Konsum häufig mit immensen Folgen verbunden ist. Alkohol entfaltet häufig eine stressreduzierende Wirkung im Körper und wird aus diesem Grund häufig konsumiert. Der Stress ist wiederum ein integraler Bestandteil unserer Lebenserfahrungen und wird sogar als sehr bedeutender Faktor bei der Entstehung und Aufrechterhaltung von alkoholbezogenen Störungen angesehen. Werden die eigenen Ressourcen und Copingstrategien für eine situationsadäquate Bewältigung einer Aufgabe als unzureichend bewertet, dann entsteht Stress. Folgt man den bekanntesten Stressmodellen, determiniert somit die subjektive Wahrnehmung des Stressors am stärksten die daraus resultierende neuroendokrine Reaktion. Insbesondere in den Entstehungsstadien einer Alkoholabhängigkeit könnte man eine höhere psychische Entspannung durch die stressreduzierende Wirkung des Alkohols vermuten. Dieser, im Sinne der Selbstmedikation verwendete Alkoholkonsum, ist als kurzfristige Problemlösungsstrategie beispielsweise bei einer Posttraumatischen Belastungsstörung häufig beobachtbar und hält die Störung ebenso aufrecht wie die ineffektiven Bewältigungsversuche anderer Art. Der Effekt wird nicht nur auf die Eigenschaften des Alkohols (Rezeptorwirkung, Alkoholdosis), sondern auch auf genetische Faktoren zurückgeführt. Stress wie Alkohol aktivieren die HypothalamusHypophysen-Nebennierenrinden-Achse (HHNA) und beeinflussen sich gegenseitig in einer hochkomplexen Art und Weise. Beispielsweise mittels peripherphysiologischer (Puls, EDA) sowie psychoendokrinologischer Parameter (Cortisol) sind die Effekte der Stresswirkung bei Alkoholabhängigen nachweisbar. Umgekehrt sind die Effekte des Alkohols auf das Stresssystem evident. Obwohl grundsätzlich große interindividuelle Unterschiede in der Reaktivität auf Stress bestehen, scheint eine gleichzeitige 
Berücksichtigung von Schweregrad der alkoholbezogenen Störung, wie sie imDSM-5 als Schweregradkontinuum vorgegeben wird, der akuten und chronischen Stressbelastungen als auch der Abstinenzdauer sehr wichtig zu sein für die zukünftige Diagnostik und effektive Behandlung von Menschen mit Alkoholgebrauchsstörungen. Angesichts der geringen Anzahl von Patienten, die sich einer Behandlung unterziehen, kann man hoffen, dass es zukünftig wichtiger wird, eine umfassende diagnostische Einschätzung der Behandlungsbedürftigkeit anhand verschiedenster Kriterien (z. B. leicht-, mittel- oder schwerkrank, hohe psychosoziale Stressbelastung, Leidensdruck, somatische Folgeschäden) vorzunehmen, als die kategoriale Unterscheidung zwischen Missbrauch und Abhängigkeit, insbesondere wenn man weiß und es aus der klinischen Praxis kennt, dass bei nicht wenigen Patienten mit Alkoholgebrauchsstörung die Grenze zwischen Missbrauch und Abhängigkeit eine fließende ist. Unter welchen, häufig stressigen, Lebensbedingungen sich bei dem Betroffenen der jeweilige Schweregrad der Störung entwickelt haben konnte, ist ebenso eine wichtige Frage wie die spezifischen Genvariationen und ihre Auswirkungen auf das Trinkverhalten und die HHNA. Vor allem schwerkranke Alkoholabhängige scheinen verzögert, länger und intensiver auf Stress zu reagieren als leicht-mittelschwerkranke. Die prolongierte Stressreaktion, die in dieser Studie gefunden wurde, könnte als Hinweis für die häufig beschriebenen Alkoholrückfälle in der frühen Abstinenzphase dienen. In diesem Zusammenhang betrachtet scheinen die pathogenen Effekte des Alkohols auf die HHNA mittels medikamentöser Therapie genauso behandelt werden zu können wie das mit Rückfall assoziierte Verlangen nach Alkohol. Insbesondere bei stresssensitiven Patienten in den frühen Abstinenzphasen kann die medikamentöse Therapie als protektives Element bei der Bewältigung von stressigen Anforderungen des alltäglichen Lebens wirken und das Rückfallrisiko reduzieren. Sie ebnet den Weg in die komplexe und effektive psychotherapeutische 
Behandlung mit Ressourcenaktivierung und Vorbereitung auf berufliche Wiedereingliederung. Auf der Basis vorhandener Ergebnisse in psychotherapeutischen Programmen zur Stressbewältigung wird der Fokus auf Selbstsicherheit, Copingskills und psychosoziale Unterstützung gelegt. Bis heute besteht aber keine gesicherte Erkenntnis darüber, welche Patienten mit einer diagnostizierten Alkoholabhängigkeit von Stressbewältigungsinterventionen profitieren und welche nicht. Allgemein kann man sagen: je klarer der Zusammenhang zwischen Stressbelastung und Alkoholrückfällen, desto größer die Wichtigkeit der Anwendung solcher Therapieprogramme. Betrachtet man die Forschungslage zum Thema Alkohol und Stress, so kann man feststellen, dass seit über 40 Jahren zahlreiche Untersuchungen existieren und von einer Forschungstradition gesprochen werden kann. Obwohl die meisten Studien zeigen konnten, dass Stress erheblichen Einfluss auf die Entstehung und Aufrechterhaltung von alkoholbezogenen Störungen ausübt, müssen auf diesem Forschungsfeld noch große Anstrengungen unternommen werden, um an erster Stelle genauere Standards für die Durchführung der Untersuchungen festzulegen. Die Studienergebnisse sind kaum generalisierbar, und nicht nur wegen der Zusammensetzung der Stichproben (Alkoholabhängige, Sozialtrinker etc.), sondern auch wegen unterschiedlicher Methoden zur Stressinduktion. Ähnlich sieht es aus im Bereich der Stressforschung im engeren Sinne. Es bestehen unterschiedliche Stressformen, die man bei einer Studie berücksichtigen sollte, insbesondere hinsichtlich der Akutheit des Stressors, sozialer Bewertung, Kontrollierbarkeit, Schwere und Art der Darbietung. Entsprechend unterschiedlich fallen bei dem jeweiligen Patienten oder der Patientengruppe die Cortisolwerte aus. Nicht alle erhöhten Cortisolwerte können potenziell als Störung bewertet werden. Die länger anhaltende Cortisolfehlregulation könnte jedoch bei Patienten mit Alkoholgebrauchsstörungen als zukünftiger Biomarker für die 
Behandlungsprognose dienen und somit die Erforschung neuer Therapiewege initiieren. Möglicherweise kann eine sorgfältige Prüfung der Stressbedingungen des Patienten und eine Überwachung des Cortisolspiegels während der Abstinenz für den Therapieprozess nützlich sein angesichts der hohen Wahrscheinlichkeit, dass die Normalisierung der HHNA-Aktivität mit der Abstinenzdauer im Zusammenhang steht. Zusammenfassend lassen sich aus den Ergebnissen der Studie konkrete Schlussfolgerungen für die klinische Praxis ableiten. An erster Stelle scheinen sowohl eine frühzeitige Einschätzung des Schweregrads der Abhängigkeit als auch der Stressverarbeitung bei den Betroffenen von Relevanz zu sein. Vor allem Schwerkranke haben ein erhöhtes Risiko für einen negativen Krankheitsverlauf. So könnten dann adäquate konzeptionelle, personelle und strukturelle Ressourcen für eine evidenzbasierte Rückfallprophylaxe zur Verfügung gestellt werden. Um unnötige Stressbelastungen und mögliche Alkoholrückfälle zu vermeiden, benötigt insbesondere diese Patientengruppe, neben einem nahtlosen Übergang von den stationären in die ambulanten Behandlungshilfen, eine intensivere suchtberaterische und psychotherapeutische Unterstützung. Bereits im Voraus sollten in dem Rahmen potenziell stressige Anliegen, wie beispielsweise Konflikte oder soziale Isolation des schwerkranken Patienten, fokussiert bearbeitet werden. Ebenso sollte im Hinblick auf eine mögliche gedankliche Weiterbeschäftigung (Grübeln) sowie auf mögliche Fluchttendenzen als negative Stressverarbeitungsformen ein besonderer Fokus gelegt werden.

Insgesamt betrachtet wird für die zukünftige Forschung eine sorgfältigere Prüfung der wechselseitigen Beeinflussung von Alkohol und Stress ebenso von Relevanz sein wie die Potenzierung der Stresseffekte durch genetische Vulnerabilität, Persönlichkeitsfaktoren sowie makro- und mikrosoziale Faktoren. Größere Studien unter kontrollierten, einheitlichen Bedingungen sind erforderlich, um der Frage nach 
den längerfristigen Auswirkungen von Stress auf Abstinenz und Rückfall bei Alkoholkranken nachzugehen. In den künftigen Studien sollten aber auch noch die Auswirkungen von Stress auf Alkoholkonsum in verschiedenen Altersgruppen sowie die Auswirkungen bei anderen Substanzabhängigkeiten untersucht werden. Schließlich wäre eine Berücksichtigung der Auswirkungen von Stress auf stoffungebundene Erkrankungen ebenfalls von Relevanz. 


\section{Literatur}

Aardal-Eriksson, E., Karlberg, B.E., Holm, A.C. (1998). Salivary cortisol - an alternative to serum cortisol determinations in dynamic function tests. Clinical Chemistry and Laboratory Medicine 36: 215-222.

Adam, E.K., Kumari, M. (2009). Assessing salivary cortisol in large-scale, epidemiological research. Psychoneuroendocrinology. 34:1423-1436.

Adinoff, B., Ruether, K., Krebaum, S., Iranmanesh, K., Wiliams, M.J. (2003). Increased salivary cortisol concentrations during chronic alcohol intoxication in a naturalistic clinical sample of men. Alcoholism: Clinical and Experimental Research, 27: 14201427.

Adinoff, B., Junghanns, K., Kiefer, F., Krishnan-Sarin, S. (2005). Suppression of the HPA axis stress-response: implications for relapse. Alcohol Clinical and Experimental Research, (29): 1351-1355.

Adinoff, B., Leonard, D., Price, J., Javors, M. A., Walker, R., Brown, E. S., et al. (2017). Adrenocortical sensitivity, moderated by ongoing stress, predicts drinking intensity in alcohol-dependent men. Psychoneuroendocrinology, 76, 67-76.

Aertgeerts, B., Buntinx, F., Ansoms, S., Fevery, J. (2001). Screening properties of questionnaires and laboratory tests for the detection of alcohol abuse or dependence in a general practice population. Br. J. Gen. Pract. 51 (464): 206-217.

Agosti, V., Levin, F. R. (2006). The effects of alcohol and drug dependence on the course of depression. Am J Addict 15, 71-75.

Agrawal, A., Narayanan, G. \& Oltmanns, T.F. (2013). Personality pathology and alcohol dependence at midlife in a community sample. Personal. Disord. 4 (1): 5561.

Aldao, A., Nolen-Hoeksema, S., Schweizer, S. (2010). Emotion-regulation strategies across psychopathology: A meta-analytic review. Clinical Psychology Review, 30, 217-237.

Aldridge-Gerry, A., A., Roesch, S.C., Villodas, F. et al. (2011). Daily Stress and Alcohol Consumption: Modeling Between-Person and Within-Person Ethnic Variation in Coping Behavior. Journal of Studies on Alcohol and Drugs; 72:125-134.

Allen, C.D., Rivier, C.L., Lee, S.Y. (2011). Adolescent alcohol exposure alters the central brain circuits known to regulate the stress response. Neuroscience. 182:162168.

Allen, A.P., Kennedy, P.J., Cryan, J.F., Dinan, T.G., Clarke, G. (2014). Biological and psychological markers of stress in humans: focus on the Trier Social Stress Test. Neuroscience Biobehavioral Rev. 38: 94-124.

Andreasson, S., Öjehagen, A. (2003). Psychosocial Treatment for Alcohol Dependence. In M. Berglund, S. Thelander, E. Jonson (Eds.), Treating Alcohol and Drug Abuse. An Evidence Based Review, (S. 43-188). Weinheim: Wiley-VCH.

AWMF-online: S3-Leitlinie. Screening, Diagnose und Behandlung alkoholbezogener Störungen. Stand 28.02.2016. https://www.awmf.org/uploads/tx_szleitlinien/076001I_S3-Leitlinie_Alkohol_2016-02.pdf 
Babor, T.F., Hofmann, M. DelBoca, F.K., Hesselbrock, V. Meyer, R.E., Dolinsky, Z.S., Rounsaville, B. (1992). Types of Alcoholics, Evidence of an empirically derived typology based on indicators of vulnerability and severity. Archives of General Psychiatry, 49: 599-608.

Back, S.E., Gros, D.F., Price, M., LaRowe, S., Flanagan, J., Brady, K.T., Davis, C., Jaconis, M., McCauley, J.L. (2015). Laboratory-induced stress and craving among individuals with prescription opioid dependence. Drug Alcohol Depend. 155: 60-67.

Banerjee, N. (2014). Neurotransmitters in alcoholism: A review of neurobiological and genetic studies. Indian Journal of Human Genetics, 20 (1), 20-31. http://doi.org/10.4103/0971-6866.132750.

Barnes, G.E., Mitic, W., Leadbeater, B., Dhami, M.K. (2009). Risk and protective factors for adolescent substance use and mental health symptoms. Can. J. of Community Menthal Health, 28 (1), 1-15.

Barnes, L. L. B., Harp, D., Jung, W. S. (2002). Reliability generalization of scores on the Spielberger State-Trait Anxiety Inventory. Educational and Psychological Measurement, 62 (4), 603-618.

Bartels, M., de Geus, E.J., Kirschbaum, C., Sluyter, F., Boomsma, D.I. (2003). Heritability of daytime cortisol levels in children. Behav. Genet.33: 421-433. doi: 10.1023/A:1025321609994.

Bartsch, A.J., Homola, G., Biller, A., et al. (2007). Manifestations of early brain recovery associated with abstinence from alcoholism. Brain. 130(Part 1): 36-47.

Becker, H.C. (2008). Alcohol Dependence, Withdrawal and Relapse. Alcohol. Res. Health; 31 (4): 348-361.

Becker, H.C. (2017). Influence of stress associated with chronic alcohol exposure on drinking. Neuropharm 122, 115-126.

Belujon, P., Grace, A.A. (2011). Hippocampus, amygdala, and stress: Interacting systems that affect susceptibility to addiction. Annals of the New York Academy of Science.1216 (1): 114-121.

Bernardy, N.C., King, A.C., Parsons, O.A., Lovallo, W.R. (1996). Altered cortisol response in sober alcoholics: An examination of contributing factors. Alcohol. 13 (5): 493-498.

Bianchi, R., Schonfeld, I.S., Laurent, E. (2015). Is it time to consider the "burnout syn drome" a distinct illness? Front Public Health. Vol. 3, Article 158.

Biedermann, J., Willens, T.E., Mick, E., Faraone, S., Spencer, T. (1998). Does attention-deficit hyperactivity disorder impact the development course of drug and alcohol abuse and dependence? Biological Psychiatry, 44: 269-273.

Biedermann, J., Mick, E., Faraone, S.V. (2000). Age-dependent decline of symptoms of attention deficit hyperactivity disorder: impact of remission definition and symptom type. Am J Psychiatry. 157: 816-818.

Birbaumer, N. \& Schmidt, R. F. (2010). Biologische Psychologie. 7. Auflage. Berlin: Springer Verlag. 
Blaine SK, Milivojevic V, Fox H et al. (2016). Alcohol effects on stress pathways: Impact on craving and relapse risk. Can. J. Psychiatry, 61 (3): 145-153.

Blaine, S.K., Sinha, R. (2017). Alcohol, stress, and glucocorticoids: from risk to dependence and relapse in alcohol use disorders. Neuropharm 122, 136-147.

Boden, J.M., Fergusson; D.M. (2011). Alcohol and depression. Addiction. 106 (5): 906-914.

Boschloo, L., Vogelzangs, N., van den Brink, W., Smit, J.H., Beekmann, A. T., Penninx, B.W. (2012). Predictors of the 2-year recurrence and persistence of alcohol dependence. Addiction, 107 (9): 1639-40.

Brady, K.T., Back, S.E., Waldrop, A.E., McRae, A.L., Anton, R.F., Upadhyaya, H.P., Saladin, M.E., Randall, K.P. (2006). Cold pressor task reactivity: predictors of alcohol use among alcohol-dependent individuals with and without comorbid posttraumatic stress disorder. Alcohol Clin. Exp. Res., 30 (6): 938-946.

Brantley, P., Waggoner, C. D., Jones, G. N. \& Rappaport, N. B. (1987). A daily stress inventory: development, reliability, and validity. Journal of Behavioral Medicine, 10: 61-74.

Breese, G.R., Chu, K., Dayas, C.V., Funk, D., Knapp, D.J., Koob, G.F., Le, A.D., O'Dell, L.E., Overstreet, D.H., Roberts, A.J., Sinha, R., Valdez, G.R., Weiss, F. (2005). Stress enhancement of craving during sobriety and the risk of relapse. Alcoholism: Clinical and Experimental Research, 29: 185-195.

Breese GR, Sinha R, \& Heilig M. (2011). Chronic alcohol neuroadaptation and stress contribute to susceptibility for alcohol craving and relapse. Pharmacol Ther, 129 (2), 149-171.

Brown, T.M. \& Fee, E. (2002). Walter Bradford Cannon. Pioneer Physiologist in Human Emotions. Am. J. Public Health. 92 (10): 1594-1595.

Brownley, K.A., Hurwitz, B.E., Schneiderman, N. (2000). Cardiovascular psychophysiology. In: Cacioppo, J.T., Tassinary, L.G., Berntson, G.G. editors. Handbook of Psychophysiology. 2nd ed. Cambridge University Press; Cambridge, England: 2000. pp. 224-264.

Brückmann, C., Di Santo, A., Karle, K.N., Batra, A., Nieratschker, V. (2016). Validation of differential GDAP1 DNA methylation in alcohol dependence and its potential function as a biomarker for disease severity and therapy outcome. Epigenetics. 11 (6): 456-463.

Bullock, K., Cservenka, A., Ray, L.A. (2017). Severity of alcohol dependence is negatively related to hypothalamic and prefrontal cortical gray matter density in heavy drinking smokers. Am. J. Drug Alcohol Abuse. 43: 281-290

Burger, M., Brönstrup, A., Pietrzik, K. (2000). Alkoholkonsum und Krankheiten (Schriftenreihe des Bundesministeriums für Gesundheit, Band 134). Baden-Baden: Nomos.

Bühringer, G., Augustin, R., Bergmann, E., Bloomfeld, K., Funk, W., Junge, B., Kraus, L., Merfert-Diete, C., Simon, R., Töppich, J. (2002). Alcohol consumption and alcohol related problems in Germany. Göttingen: Hogrefe Verlag. 
Cadoret, R.J., Yates, W.R., Troughton, E., Woodworth, G. \& Steward, M.A. (1995). Adoption study demonstrating two genetics pathways to drug abuse. Arch. Gen. Psychiatry, 52: 42-52.

Carpenter LL, Shattuck TT, Tyrka AR, Geracioti TD, Price LH (2011). Effect of childhood physical abuse on cortisol stress response. Psychopharmacology (Berl) 2011; 214: 367-75.

Chambless, D. L., Ollendick, T. H. (2001). Empirically supported psychological interventions: controversies and evidence. Annual review of psychology. 52: 685-716.

Chaplin, T.M., Hong, K., Fox, H.C., Siedlarz, K.M., Bergquist, K., Sinha, R. (2010). Behavioral arousal in response to stress and drug cue in alcohol and cocaine addicted individuals versus healthy controls. Hum. Psychophamacol. 25 (5): 368-376.

Charmandari, E., Tsigos, C., \& Chrousos, G. (2005). Endocrinology of the stress response. Annu. Rev. Physio. 67, 259-284.

Chow, C.M., Wieman, D., Cichocki, B., Qvicklund, H., Hiersteiner, D. (2012): Mission impossible: treating serious mental illness and substance use co-occurring disorder with integrated treatment: a metaanalysis. Mental Health and Substance Use 1: 1-19.

Cloninger, C.R., Bohmann, M., \& Sigvardsson, S. (1981). Inheritance of alcohol abuse: cross-fostering analysis of adopted men. Arch. Gen. Psychiatry, 38: 861-868.

Clow A. (2004). Cortisol as a biomarker of stress. J Holist Healthc. 1: 10-14.

Clow, A., Hucklebridge, F., Stalder, T., Evans, P., Thorn, L. (2010). The cortisol awakening response: More than a measure of HPA axis function. Neurosci. Biobehav. Rev. 35: 97-103.

Coffey, S.F., Stasiewicz, P.R., Hughes, P.M., Brimo, M.L. (2006). Trauma-focused imaginal exposure for individuals with comorbid posttraumatic stress disorder and alcohol dependence: revealing mechanisms of alcohol craving in a cue reactivity paradigm. Psych. Addict. Behav. 20: 425-435.

Cohen, S., Frank, E., Doyle, W.J., Skoner, D.P., Rabin, B.S., Gwaltney, J.M. (1998). Types of stressors that increase susceptibility to the common cold in healthy adults. Health Psychol.17: 214-223.

Coiro, V., Casti, A., Jotti, G.S., et al. (2007). Adrenocorticotropic hormone/cortisol response to physical exercise in abstinent alcoholic patients. Alcoholism: Clinical and Experimental Research. 31 (5): 901-906.

Conger, J.J. (1956). Alcoholism: Theory, problem and challenge, II: Reinforcement theory and the dynamics of alcoholism. Quarterly Journals of Studies on Alcohol, 54, 326-333.

Conner, K.R., Pinquart, M., Gamble, S.A. (2009). Meta-analysis of depression and substance use among individuals with alcohol use disorders. J Subst Abuse Treat. 37: 127-37.

Crabbe, J. \& Lovinger, D.M. (2005). Animal models of alcoholism: treatment target identification and insight into mechanisms. Nature Neuroscience. 8, 1471-1480. 
Critchley, H. D. (2002). Electrodermal Responses: What Happens in the Brain? The Neuroscientist, 8 (2): 132-142.

Dani, J.A., Harris, R.A. (2005). Nicotine addiction and comorbidity with alcohol abuse and mental illness. Nature Neuroscience. 8: 1465-1470.

Dantzer, R. (2001). Cytokine-induced sickness behavior: Where do we stand? Brain Behav. Immun. 15: 7-24.

Dawson, D.A., Grant, B.F., Ruan, W.J. (2005). The association between stress and drinking: modifying effects of gender and vulnerability. Alcohol and Alcoholism 40: 453-460.

Dawson, M.E., Schell, A.M., Filion, D.L. (2007). The electrodermal system. In: Cacioppo JT, Tassinary LG, Berntson GG, editors. Handbook of Psychophysiology. 3rd edition. New York: Cambridge University Press; 2007. pp. 159-181.

Debell, F., Fear, N.T., Head, M., Batt-Rawden, S., Greenberg, N., Wessely, S., Goodwin, L. A. (2014). A systematic review of the comorbidity between PTSD and alcohol misuse. Soc Psychiatry Psychiatr Epidemiol. 49: 1401-1425.

Dedic, N., Chen, A., Deussing, J.M. (2018). The CRF family of neuropeptides and their receptors - mediators of the central stress response. Current Molecular Pharmacology. 11; 4-31.

Deister, A. (2005). Beschäftigungstherapie, Arbeitstherapie, Kunsttherapie, Musiktherapie. In H.J. Möller, G. Laux, H.P. Kapfhammer (Hrsg.). Psychiatrie und Psychotherapie (S. 806-820). Berlin: Springer.

de Kloet, E.R. (2004). Hormones and the stressed brain. Ann N Y Acad Sci. 1018: $1-15$.

de Kloet, E.R., Joëls, M. \& Holsboer, F. (2005). Stress and the brain: from adaptation to disease. Nature Reviews Neuroscience, 6: 463-475.

de Kloet, E.R., Meijer, O.C., de Nicola, A.F., de Rijk, R.H., Joëls, M. (2018). Importance of the brain corticosteroid receptor balance in metaplasticity, cognitive performance and neuro-inflammation. Frontiers in Neuroendocrinology 49: 124-145. (https://doi.org/10.1016/j. yfrne.2018.02.003).

Demmel, R., Rist, F. \& Olbrich, R. (2000). Autonomic reactivity to mental stressors after single administration of lorazepam in male alcoholics and healthy controls. Alcohol and Alcoholism, 35, 617-624.

Deutsche Hauptstelle für Suchtfragen (DHS) e. V., (2018). Jahrbuch Sucht 2018. Pabst Science Publishers. 1. Auflage 2018.

Dickerson, S.S. \& Kemeny, M.E. (2004). Acute stressors and cortisol responses: a theoretical integration and synthesis of laboratory research. Psychological Bulletin, 130 (3): 355-391.

Dilling, H., Mombour, W., Schmidt, M.H. (1993). Internationale Klassifikation psychischer Störungen. ICD-10 Kapitel V (F), 2. Auflage, Huber, Bern. 
Diorio, J., \& Meaney, M. J. (2007). Maternal programming of defensive responses through sustained effects on gene expression. Journal of Psychiatry \& Neuroscience, 32 (4), 275-284.

Ditzen, B. \& Nater, U. M. (2006). Rezension - P. Schulz, W. Schlotz \& P. Becker (2004). Trierer Inventar zum chronischen Stress (TICS). Göttingen: Hogrefe.

Driessen, M. (2004). Psychiatrische Komorbidität (DSM-VI - Achsen I und II) und Suizidalität bei Alkoholabhängigkeit. Suchtmed 6 (2): 138.

Driessen, M., Schulte, S., Luedecke, C., Schaefer, I., Sutmann, F., Ohlmeier, M., Kemper, U., Koesters, G., Chodzinski, C., Schneider, U., Broese, T., Dette, C., Havemann-Reinicke, U. (2008). Trauma and PTSD in patients with alcohol, drug, or dual dependence: a multi-center study. Alcohol Clin Exp Res. 32: 481-488.

Durham, RC., Chambers, J.A., Power, K.G., Sharp, D.M., MacDonald, R.R., et al. (2005). Long-term outcome of cognitive behaviour therapy clinical trials in central Scotland. Health Technol Assess. 5; 9 (42):1-174.

Edwards, G., Gross, M. (1976). Alcohol dependence: provisional description of a clinical syndrome. Br. Med J.; 1: 1058-1061.

Edwards, G. (1997). Alcohol policy and the public good. Addiction. 1: 73-79.

Egle, U.T., Hardt, J., Nickel, R., Kappis, B., Hoffmann, S.O. (2002). Früher Stress und Langzeitfolgen für die Gesundheit - Wissenschaftlicher Erkenntnisstand und Forschungsdesiderate. Z. für Psychosomatische Medizin u. Psychotherapie, 48 (4): 411-434.

Egle, U.T., Hoffmann, S.O., Steffens, M. (1997). Psychosoziale Schutzfaktoren in Kindheit und Jugend als Prädisposition für psychische Störungen im Erwachsenenalter. Nervenarzt. 68: 683-695.

Egle, U., T., Hoffmann, S.O., Joraschky, P. (2015): Sexueller Missbrauch, Misshandlung, Vernachlässigung: Erkennung, Therapie und Prävention der Folgen früher Stresserfahrungen. 4. Aufl. Stuttgart: Schattauer.

Ehrenreich, H., Schuck, J., Stender, N., Pilz, J., Gefeller, O., Schilling, L., Poser, W., Kaw, S. (1997). Endocrine and hemodynamic effects of stress versus systematic CRF in alcoholics during early and medium term abstinence. Alcoholism: Clinical and Experimental Research, 21: 1285-1293.

Eisenbarth, H., Chang, L.J., Wager, T.D. (2016). Multivariate Brain Prediction of Heart Rate and Skin Conductance Response to Social Threat. Journal of Neuroscience. 36: 11987-11998.

Ellickson, S.L., Tucker, J.S., Klein, D.J., McGuigan, K.A. (2001). Prospective risk factors for alcohol misuse in late adolescence. Journal of Studies on Alcohol. (6): 773-782.

Enoch, M.A. (2011). The Role of Early Life Stress as a Predictor for Alcohol and Drug Dependence. Psychopharmacology, 214 (1): 17-31.

Enoch, M.A. (2013). Genetic Influences on the Development of Alcoholism. Curr. Psychiatry Rep., 15 (11): 412. 
Erdmann, G., Janke, W. (2008). Stressverarbeitungsfragebogen (SVF). 4 Auflage, Göttingen, Hogrefe Verlag.

Eriksen, H.R. \& Ursin, H. (2004). Subjective health complaints, sensitization, and sustained cognitive activation (stress). Journal of Psychosomatic Research, 56, 445448.

Falkai P., Wittchen H.U. (2015). Diagnostisches und Statistisches Manual Psychischer Störungen DSM-5. Göttingen: Hogrefe.

Faraone, S.V., Biederman, J., Mick, E. (2006). The age-dependent decline of attention deficit hyperactivity disorder: a meta-analysis of follow-up studies. Psychol. Med. 36:159-165.

Farber, B.A. (2000). Understanding and Treating Burnout in a Changing Culture. JCLP/In Session. Psychotherapy in Practice. 56: 589-594.

Feder, A., Nestler, E. J., Charney D. S. (2009). Psychobiology and molecular genetics of resilience. Nature Review Neuroscience. 10, 446-457. 10.1038/nrn2649

Federenko, I.S., Nagamine, M., Hellhammer, D.H., Wadhwa, P.D., Wüst, S. (2004). The Heritability of Hypothalamus Pituitary Adrenal Axis Responses to Psychosocial Stress Is Context Dependent. Journal of Clinical Endocrinology \& Metabolism, 89 (12): 6244-6250.

Fein, G. (2015). Psychiatric Comorbidity in Alcohol Dependence. Neuropsychol. Rev. 25 (4): 456-475.

Felitti, V. J., Anda, R. F., Nordenberg, D., et al. (1998). Relationship of childhood abuse and household dysfunction to many of the leading causes of death in adults: the adverse childhood experiences (ACE) study. American Journal of Preventive Medicine. 14 (4): 245-258.

Fox, H.C., Bergquist, K.L., Hong, K et al. (2007). Stress-induced and alcohol cueinduced craving in recently abstinent alcohol dependent individuals. Alcohol Clin. Exp. Res.; 31: 395-403.

Gardner, E.L. (2011). Addiction and brain reward and antireward pathways. Adv. Psychosom. Med. 30: 22-60.

George, C.L., Birnie, M.T., Flynn, B.P., Kershaw, Y.M., Lightman, S.L., ConwayCampbell, B.L. (2017). Ultradian glucocorticoid exposure directs gene-dependent and tissue-specific mRNA expression patterns in vivo. Molecular and Cellular Endocrinology, 439; 46-53.

Geyer, D., Batra, A., Beutel., M., Funke, W. et al. (2006). AWMF-Leitlinie: Postakutbehandlung alkoholbezogener Störungen. Sucht; Zeitschrift für Wissenschaft und Praxis. 52 (1), 8-34.

Gillberg C, Gillberg IC, Rasmussen P, Kadesjö B, Söderström H, Råstam M, et al. (2004). Co-existing disorders in ADHD-implications for diagnosis and intervention. Eur Child Adolesc Psychiatry. 13(1): 80-92.

Gilpin, N.W., Weiner, J.L. (2017). Neurobiology of comorbid post-traumatic stress disorder \& alcohol-use disorder. Genes, Brain and Behavior. 16 (1):15-43. 
Glas, J.E., Williams, E.C. \& Buchholz, K.K. (2014). Psychiatric comorbidity and perceived alcohol stigma in a nationally representative sample of individuals with DSM5 alcohol use disorder. Alcohol. Clin. Exp. Res. 38 (6): 1697-1705.

Goodwin, D.W. (1992). Genetic determinants of alcoholism. In: Mendelson, J.H. \& Mello, N.K. (Hrsg): Medical Diagnosis and Treatment of Alcoholism. McGraw-Hill, New York, 1992: 55-70.

Gozansky, W.S., Lynn, J.S., Laudenslager, M.L., Kohrt, W.M. (2005). Salivary cortisol determined by enzyme immunoassay is preferable to serum total cortisol for assessment of dynamic hypothalamic-pituitary-adrenal axis activity. Clinical Endocrinology. 63(3): 336-341.

Grant, B.F., Hasin, D.S., Chou, S.P., Stinson, F.S. \& Dawson, D.A. (2004a). Nicotine dependence and psychiatric disorders in the United States: results from the national epidemiologic survey on alcohol and related conditions. Arch. Gen. Psychiatry 61, 1107-1115.

Grant, B.F., Stinson, F.S., Dawson, D.A., Chou, S.P., Ruan, W.J., Pickering, R.P. (2004b). Coocurrence of 12-month alcohol and drug use disorders and personality disorders in the United States: results from the National Epidemiologic Survey on Alcohol and Related Conditions. Arch. Gen. Psychiatry, 61: 361-368.

Gsellhofer, B., Fahrner, E-M., (1994). Manual für Training und Durchführung von Interviews mit dem EuropAsi (Deutsche Version). München, Institut für Therapieforschung (IFT), Vervielfältigtes Manuskript.

Guo, R., Ren, J. (2010). Alcohol and acetaldehyde in public health: from marvel to menace. Int J Environ Res Public Health. 7: 1285-1301.

Habib, K. E., Gold, P. W., \& Chrousos, G. P. (2001). Neuroendocrinology of stress. Endocrinol. Metab. Clin. North. Am. 30 (3),695-728.

Hadley, M.E., Haskell-Luevano, C. (1999). The proopiomelanocortin system. Ann N Y Acad Sci. 885: 1-21.

Hall, L.H., Johnson, J., Watt, I., Tsipa, A., O'Connor, D.B. (2016). Healthcare staff wellbeing, burnout, and patient safety: a systematic review. PLoS One. 2016; 11: e0159015. doi: 10.1371/

Hammen, C. (2005). Stress and depression. Annu. Rev. Clin. Psychol. 1: 293-319.

Hapke, U., Riedel, J., Thyrian, J.R., Röske, K., Michael, A., Wosniak, R. Does, D., Ohde, L., John, U. (2003). Schwerpunktpraxen Sucht: Ein Bindeglied zwischen Suchttherapie und medizinischer Basisversorgung. In H.-J. Rumpf \& R. Hüllighorst (Hrsg.). Alkohol und Nikotin: Frühintervention, Akutbehandlung und politische Maßnahmen (S. 344-357). Freiburg: Lambertus Verlag.

Harbuz, M.S., Chover-Gonzalez, A.J., Jessop, D.S. (2003). Hypothalamo-pituitaryadrenal axis and chronic immune activation. Ann. NY Acad. Sci. 992: 99-106.

Harwood, D.M., Hawton, K., Hope, T., Harris, L., Jacoby, R. (2006). Life problems and physical illness as risk factors for suicide in older people: a descriptive and casecontrol study. Psychological Medicine. 36, 1265-1274.

Hautzinger, M., Bailer, M., Worall, H. \& Keller, F. (1994). Beck-Depressions-Inventar (BDI). Bern: Huber. 
Hautzinger, M., Keller, F., Kühner, C. (2009). Becks-Depressions-Inventar-II, (BDI-II). Revision. Originalbezug Testzentrale Göttingen, Hogrefe Verlag.

Heather, N. \& Robertson, I. (1997). Problem drinking. 3. Edition. Oxford University Press. Oxford.

Heim, C., Shugart, M., Craighead, W.E., Nemeroff, C.B. (2010). Neurobiological and psychiatric consequences of child abuse and neglect. Dev Psychobiol. 2010;52: 671690.

Heinz, A.J., Beck, A., Meyer-Lindenberg, A., Sterzer, P., Heinz, A. (2011). Cognitive and neurobiological mechanisms of alcohol-related aggression. Nature Reviews Neuroscience, 12: 400-413.

Hellhammer, D.H., Wüst, S., Kudielka, B.M. (2009). Salivary cortisol as a biomarker in stress research. Psychoneuroendocrinology. 34: 163-171.

Henkel, D. (2011). Unemployment and substance use: a review of the literature (19902010). Curr Drug Abuse Rev, 4: 4-27

Herman, J.P., Cullinan, W.E. (1997). Neurocircuitry of stress: Central control of the hypothalamo-pituitary-adrenocortical axis. Trends in Neuroscience. 20(2):78-84.

Herman, J.P., McKlveen, J.M., Ghosal S., Kopp, B., Wulsin, A., Makinson, R., Scheimann, J., Myers, B. (2016). Regulation of the hypothalamic-pituitaryadrenocortical stress response. Comprehensive Physiology. 6: 603-621. (https://doi.org/10.1002/cphy.c150015).

Herzberg, P.Y., Goldschmidt, S., Heinrichs, N. (2008). Beck Depressions-Inventar (BDI-II). Revision. Report Psychologie. (6) 33: 301-302.

Ho, P.S., Shih, M.C., Ma, K.H., et al. (2011): Availability of the serotonin transporter in patients with alcohol dependence. World J. Biological Psychiatry, 12: 134-142.

Holmes, T. H. \& Rahe, R.H. (1967). The social readjustment rating scale. Journal of Psychosomatic Research, 11, 213-218.

Horvath, A. O., Luborsky, L. (1993). The role of the therapeutic alliance in psychotherapy. Journal of Consulting and Clin. Psychol. 61: 561-573.

Horvath, A.O., Del Re, A.C., Flückiger, C., Symonds, D. (2011). Alliance in individual psychotherapy. Psychotherapy. 48(1): 9-16.

Hurt, R.D., Dale, L.C., Offord, K.P., Croghan, I.T., Hays, J.T., Gomez-Dahl, L. (1995). Nicotine patch therapy for smoking cessation in recovering alcoholics. Addiction 90: 1541-1546.

Inoué, H., Stickel, F., Seitz, H.K. (2001). Individuelles Risikoprofil bei chronischem Alkoholkonsum. Aktuelle Ernährungs Med. 26: 39-46.

Inskip, H.M., Harris, E.C., Barraclough, B. (1998). Lifetime risk of suicide for affective disorder, alcoholism and schizophrenia. Br J Psychiatry. 172:35-37.

Isaacson, J.H., Butler, R., Zacharek, M., Tzelepis, A. (1994). Screening with the Alcohol use Disorders Identification Test (AUDIT) in an inner-city population. J. Gen. Intern. Med. (10): 550-553. 
Irwin, M., Schuckit, M. \& Smith, T.L. (1990). Clinical importance of age of onset in type 1 and type 2 primary alcoholics. Arch. Gen. Psychiatry, 47: 320-324.

Jacobi, C., Brand-Jacobi, J., Marquardt, F. (1987). Die „Göttinger Abhängigkeitsskala (GABS)": ein Verfahren zur differentiellen Erfassung der Schwere der Alkoholabhängigkeit. Suchtgefahren; 33: 23-26.

Joëls, M. (2018). Corticosteroids and the brain. Journal of Endocrionology. 238, R121-R130. Rev.

John, U., Hapke, U., Rumpf, H-J. (2001). Skala zur Erfassung der Schwere der Alkoholabhängigkeit (SESA). Göttingen, Hogrefe Verlag.

Johnson, B. A. (2008). Update on neuropharmacological treatments for alcoholism: scientific basis and clinical findings. Biochemical pharmacology. 75, 34-56.

Johnson, J.G, Cohen, P., Kasen, S., Skodol, A.E., Oldham, J.M. (2008). Cumulative prevalence of personality disorders between adolescence and adulthood. Acta Psychiatr. Scand. 118: 410-413.

Jonas, D.E., Amick, H.R., Feltner, C., Bobashev, G., Thomas, K., Wines, R., et al. (2014). Pharmacotherapy for adults with alcohol use disorders in outpatient settings: a systematic review and meta-analysis. JAMA 311: 1889-1900.

Jung, M.H., Park, B.L., Lee, B.C., Ro, Y., Park, R., Shin, H.D., Bae, J.S., Kang, T.C. \& Choi, I.G. (2011). Association CHRM2 polymorphisms with severity of alcohol dependence. Genes, Brain and Behavior, 10 (2): 253-256.

Junghanns, K., Backhaus, J., Tietz, U. (2003). Impaired serum cortisol stress response is a predictor of early relapse. Alcohol \& Alcoholism, 38: 189-193.

Junghanns, K., Tietz, U., Dibbelt, L., Kuether, M., Jurth, R., Ehrenthal, D., Blank, S., Backhaus, J. (2005). Attenuated salivary cortisol secretion under cue exposure is associated with early relapse. Alcohol \& Alcoholism, 40 (1): 80-85.

Junghanns, K., Horbach, R., Ehrenthal, D., et al. (2007). Cortisol awakening response in abstinent alcohol-dependent patients as a marker of HPA-axis dysfunction. Psychoneuroendocrinology,32(8-10): 1133-1137.

Juster, R.P., Sindi S., Marin M.F., Perna A., Hashemi A., Pruessner J.C., Lupien S.J. (2011). A clinical allostatic load index is associated with burnout symptoms and hypocortisolemic profiles in healthy workers. Psychoneuroendocrinology. 36: 797805.

Kadden, R.M., Litt, M.D., Cooney N.L., Kabela E., Getter H. (2001). Prospective matching of alcoholic clients to cognitive-behavioral or interactional group therapy. J Stud Alcohol. 62 (3): 359-369.

Kalivas, P.W., O'Brien, C. (2008). Drug addiction as a pathology of staged neuroplasticity. Neuropsychopharmacology. 33: 166-180.

Kaltsas, G.A. \& Chrousos, G.P. (2007). The Neuroendocrinology of Stress. In Cacioppo, J.T., Tassinary, L.G., Berntson, G.G., editors. Handbook of Psychophysiology. 3rd edition. New York: Cambridge University Press; Chapter 13. pp. 303-318. 
Kapoor, M., Chou, Y-L., Edenberg, H.J., Foroud, T., Martin, N.G., Madden, P.A.F., Wang, J.C., Bertelsen, S., Wetheril, L., Brooks, A., Chan, G., Hesselbrock, V., Kuperman, S., Medland, S.E., Montgomery, G., Tischfield, J., Whitfield, J.B., Bierut, L.J., Heath, A.C., Buchholz, K.K., Goate, A.M. \& Agrawal, A. (2016). Genome-wide polygenic scores for age at onset of alcohol dependence and association alcoholrelated measures. Transl. Psychiatry, 6, e761.

Kelly, T.M., Lynch, K.G., Donovan, J.E., Clark, D.B. (2001). Alcohol use disorders and risk factor interactions for adolescent suicidal ideations and attempts. Suicide and LifeThreatening Behavior. 31, 181-193.

Kemeny, M.E., Schedlowski, M. (2007). Understanding the interaction between psychosocial stress and immune-related diseases: a stepwise progression. Brain Behav. Immun. 21: 1009-1018.

Kendler, K.S., Karkowski, L.M., Prescott, C.A. (1999). Causal relationship between stressful life events and the onset of major depression. Am. J. Psychiatry. 156: 837841.

Kendler, K.S., Myers, J., Prescott, C.A. (2000). Parenting and adult mood, anxiety and substance use disorders in female twins: An epidemiological, multi-informant, retrospective study. Psychological Medicine. 30: 281-294.

Keyes, K.M., Hatzenbuehler, M.L., Hasin, D.S. (2011). Stressful life experiences, alcohol consumption, and alcohol use disorders: the epidemiologic evidence for four main types of stressors. Psychopharmacology. 218 (1): 1-17.

Kiecolt-Glaser, J.K., McGuire, L., Robles, T.F., Glaser, R. (2002). Psychoneuroimmunology: psychological influences on immune function and health. J. Consult. Clin. Psychol. 70: 537-547.

Kiefer, F., Jahn, H., Schick, M. et al. (2002). Alcohol selfadministration, craving and HPA-axis activity: an intriguing relationship. Psychopharmacology (Berl) 164:239240.

Kiefer, F.; Jahn, H.; Otte, C.; Naber, D.; Wiedermann, K. (2006). Hypothalamicpituitaryadrenocortical axis activity: a target of pharmacological anti-craving treatment? Biol. Psychiatry 60: 74-76.

Kienast, T., Heinz, A. (2006). Dopamine and the diseased brain. CNS \& Neurological Disorders - Drug Targets 5, 109-131.

Kienast, T., Hariri, A.R., Schlagenhauf, F., Wrase, J., Sterzer, P., Buchholz, H.G., Smolka, M.N., Gründer, G., Cumming, P., Kumakura, Y., Bartenstein, P., Dolan, R.J., Heinz, A. (2008). Dopamine in amygdala gates limbic processing of aversive stimuli in humans. Nature Neuroscience, 11: 1381-1382.

King, A.C., Bernardy, N.C., Hauner, K. (2003). Stressful events, personality and mood disturbance: gender differences in alcoholics and problem drinkers. Addict Behav 28; 171-187.

Kirschbaum, C., \& Hellhammer, D. H. (1999). Hypothalamus-Hypophysen-Nebennierenrindenachse. In Enzyklopädie der Psychologie. Psychoendokrinologie und Psychoimmunologie (Bd. 3). Göttingen, Bern, Toronto, Seattle: Hogrefe. 
Kirschbaum, C., Pirke, K.M., Hellhammer, D.H. (1993). The Trier Social Stress Test a tool for investigating psychobiological stress responses in a laboratory setting. Neuropsychobiology, 28 (1-2): 76-81.

Kirschbaum, C. \& Hellhammer, D.H. (1994). Salivary cortisol in psychoneuroendocrine reserach: recent developments and applications. Psychoneuroendocrinology 19: 313-333.

Kirschbaum, C. (1991). Cortisolmessung im Speichel - Eine Methode der Biologischen Psychologie. Bern, Göttingen, Toronto: Huber.

Klengel, T., Mehta D., Anacker C., Rex-Haffner M., Pruessner J. C., Pariante C. M., et al. . (2013). Allele-specific FKBP5 DNA demethylation mediates gene-childhood trauma interactions. Nature Neuroscience. 16 (1), 33-41.

Klingemann H, Gmel G. (2001). Mapping the social consequences of alcohol consumption. Dordrecht, Kluwer Academic Publishers.

Koller G., Preus, U.W., Bottlender, M., Wenzel, K., Soyka, M. (2002). Impulsivity and aggression as predictors of suicide attempts in alcoholics. European Archives of Psychiatry and Clinical Neuroscience. 252, 155-160.

Koob, G.F. (2008). A Role of brain stress systems in addiction. Neuron, 59 (1) 11-34. Koob, G.F., Volkow, N.D. (2010). Neurocircuitry of addiction. Neuropsychopharmacology. 35: 217-38.

Koob G.F., Buck, C.L., Cohen, A., Edwards, S., Park, P.E., Schlosburg, J.E., Schmeichel, B., Vendruscolo, L.F., Wade, C.L., Whitfield, T.W., Jr., George, O. (2014). Addiction as a stress surfeit disorder. Neuropharmacology. 76: 370-382.

Koolhaas, J.M., Bartolomucci, A., Buwalda B., de Boer, S.F., Flügge, G. Korte, S.M, Meerlo, P., Murison, R., Olivier, B., Palanza, P., Richter-Levin, G., Sgoifo, A., Steimer, T., Stiedl, O., van Dijk, G., Wöhr, M. \& Fuchs, E. (2011). Stress Revisited: a critical evaluation of the stress concept. Neurosci. Biobehav. Rev. 35 (5): 1291-1301.

Krantz, D.S., McCeney, M.K. (2002). Effects of Psychological and Social Factors on Organic Disease: A Critical Assessment of Research on Coronary Heart Disease. Annu. Rev. Psychol. 53: 341-369.

Kruse, G., Körkel, J. \& Schmalz, U. (2000). Alkoholabhängigkeit erkennen und behandeln. Bonn: Psychiatrie Verlag.

Kudielka, B.M. \& Kirschbaum, C. (2005). Sex differences in HPA axis responses to stress: a review. Biol. Psychology, 69 (1): 113-132.

Kudielka, B. M., Hellhammer, D. H., \& Kirschbaum, C. (2007). Ten years of research with the Trier Social Stress Test - Revisited. In Social Neuroscience: Integrating Biological and Psychological Explanations of Social Behavior (S. 56-83). New York: Guilford Press.

Kudielka, B.M., Hellhammer, D.H. \& Wüst, S. (2009). Why do we respond so differently? Reviewing determinants of human salivary cortisol responses to challenge. Psychoneuroendocrinology, 34 (1): 2-18.

Küfner, H. \& Kraus, L. (2002). Alkoholismus: Epidemiologische und ökonomische Aspekte des Alkoholismus. Deutsches Ärzteblatt, 99, 936-945. 
Lai, H.M., Cleary, M., Sitharthan, T., Hunt, G.E. (2015). Prevalence of comorbid substance use, anxiety and mood disorders in epidemiological surveys, 1990-2014: systematic review and meta-analysis. Drug Alcohol Depend 154, 1-13.

Landsbergis, P.A., Grzywacz, J.G. \& La Montagne, A.D. (2014). Work organization, job insecurity and occupational health disparities. American Journal of Industrial Medicine, 57 (5): 495-515.

Laux, L., Glanzmann, P., Schaffner, P., Spielberger, C.D. (1981). State-TraitAngstinventar (STAl): Deutsche Version. Göttingen, Hogrefe Verlag.

Lazarus, R.S. \& Folkmann, S. (1984). Stress, appraisal, and coping. New York: Springer.

Lieb, R., Isensee, B. (2002). Häufigkeit und zeitliche Muster von Komorbidität. In: Moggi, F. (Hrsg.): Doppeldiagnosen. Komorbidität psychischer Störungen und Sucht. Bern: Huber 2002, 31-62

Liebermann, R., Kranzler, H.R., Joshi, P., Shin, D.G. \& Covault, J. (2015). GABRA2 Alcohol Dependence Risk Allele is Associated with Reduced Expression of Chromosome 4p12 GABAA Subunit Genes in Human Neural Cultures. Alcohol. Clin. Exp. Res., 39 (9): 1654-64.

Litt, M.D., Kadden, R.M., Kabela-Cormier, E. (2009). Individualized assessment and treatment program for alcohol dependence: results of an initial study to train coping skills. Addiction, 104(11), 1837-1838.

Lovallo, W.R., Dickensheets, S.L., Myers, D.A., Thomas, T.L., Nixon, S.J. (2000). Blunted stress cortisol response in abstinent alcoholic and polysubstance-abusing men. Alcoholism: Clinical and Experimental Research, 24: 651-658.

Lovallo W. R., Farag N. H., Sorocco K. H., Cohoon A. J., Vincent A. S. (2012). Lifetime adversity leads to blunted stress axis reactivity: studies from the Oklahoma family health patterns project. Biol. Psychiatry. 71, 344-349.

Lupien, S.J., de Leon, M., de Santi, S., Convit, A., Tarshish, C., Nair, N.P., Thakur, M., McEwen, B.S., Hauger, R.L., Meaney, M.J. (1998). Cortisol levels during human aging predict hippocampal atrophy and memory deficits. Nature Neuroscience. 1:69-73.

Lupien, S. J., McEwen, B. S., Gunnar, M. R., \& Heim, C. (2009). Effects of stress throughout the lifespan on the brain, behaviour and cognition. Nature Reviews Neuroscience, 10 (6), 434-445.

Magrys, S., Olmstead, M., Wynne-Edwards, K., Balodis, I. (2013). Neuroendocrinological responses to alcohol intoxication in healthy males:relationship with impulsivity, drinking behavior, and subjective effects. Psychophysiology. 50:204209.

Maier W., Falkai P., Heinz A: Stellungnahme der Deutschen Gesellschaft für Psychiatrie und Psychotherapie, Psychosomatik und Nervenheilkunde (DGPPN). Wann wird seelisches Leiden zur Krankheit? Zur Diskussion um das angekündigte Diagnosesystem. DSM-V.www.dgppn.de/fileadmin/user_apload/_medien/download/ $\mathrm{pdf} /$ stellungnahmen/2013DGPPN-Stellungnahme_DSM-5_Final.pdf (aufgerufen 27.11.2015). 
Mann, K. (2010). Suchtforschung und Suchttherapie - eine Vision für 2020. Sucht, 56 (1), 9-10.

Mann, K., Bladstrom, A., Torup, L., Gual, A., van den Brink W. (2013). Extending the treatment options in alcohol dependence: a randomized controlled study of as-needed nalmefene. Biol Psychiatry. 73(8):706-713.

Marinelli, M., Piazza, P. V. (2002). Interaction between glucocorticoid hormones, stress and psychostimulant drugs. Eur. J. Neurosci. 16 (3), 387-394.

Martinek, L., Oberascher-Holzinger, K., Weishuhn, S., Klimesch, W., Kerschbaum, H.H. (2003). Anticipated academic examinations induce distinct cortisol responses in adolescent pupils. Neuro Endocrinol. Lett. 24(6):449-453.

Mason, B.L., Pariante, C.M., Jamel, S., Thomas, S.A. (2010). Central nervous system (CNS) delivery of glucocorticoids is fine-tuned by saturable transporters at the bloodCNS barriers and nonbarrier regions. Endocrinology. 151(11):5294-5305.

Mason, B.L., Pariante, C.M., Thomas, S.A. (2008). A revised role forP-glycoprotein in the brain distribution of dexamethasone, cortisol, and corticosterone in wild-type and ABCB1A/B-deficient mice. Endocrinology. 149:5244-5253.

Massie, M.J. (2004). Prevalence of depression in patients with cancer. J Natl Cancer Inst Monogr. 32: 57-71.

Mattoo, S.K., Chakrabati, S., Anjaiah, M. (2009). Psychosocial factors associated with relapse in men with alcohol or opioid dependence. Indian J. Med. Res. 130: 702-708.

McCarthy, E., \& Petrakis, I. (2010). Epidemiology and management of alcohol dependence in individuals with post-traumatic stress disorder. CNS Drugs, 24 (12): 997-1007.

McEwen B.S. (1998). Protective and damaging effects of stress mediators. New England Journal of Medicine 338 171-179.

McEwen, B.S., Wingfield, J.C. (2003). The concept of allostasis in biology and biomedicine. Hormones and Behavior 43: 2-15.

McEwen, B.S. (2004). Protection and damage from acute and chronic stress: allostasis and allostatic overload and relevance to the pathophysiology of psychiatric disorders. Annals of the New York Academy of Sciences, 1032 (1), 17. http://dx.doi.org/10.1196/annals.1314.001.

McGovern, M.P., Lambert-Harris, C., McHugo, G.J., Giard, J., Mangrum, L. (2010). Improving the dual diagnosis capability of addiction and mental health treatment services: Implementation factors associated with program level changes. Journal of Dual Diagnosis. 6: 237-250.

McGowan P. O., Sasaki A., D’Alessio A. C., Dymov S., Labonte B., Szyf M., Turecki, G., Meany, M. J. (2009). Epigenetic regulation of the glucocorticoid receptor in human brain associates with childhood abuse. Nature Neuroscience. 12: 342-348.

McLellean A.T., Druley K.A., O'Brien C.P., Kron R. (1980). Matching substance abuse patients to appropriate treatments. A conceptual and methodological approach. Drug and Alcohol Dependence, 5 (3), 189-193. 
Meyerhoff, D.J., Blumenfeld, R., Truran, D., Lindgren J., Flenniken, D., Cardenas, V., et al. (2004). Effects of heavy drinking, binge drinking, and family history of alcoholism on regional brain metabolites. Alcoholism: Clinical and Experimental Research. 28(4):650-661.

Miller, D.B., \& O'Callaghan, J.P. (2002). Neuroendocrine aspects of the response to stress. Metabolism 51: 5-10.

Miller, G.E., Chen, E., Zhou, E.S. (2007). If it goes up, must it come down? Chronic stress and the hypothalamic-pituitary-adrenocortical axis in humans. Psychol. Bull. 133: $25-45$.

Miller, W. R. Rollnick, S. (1999). Motivierende Gesprächsführung. Ein Konzept zur Beratung von Menschen mit Suchtproblemen. Freiburg: Lambertus.

Miller, W. R., \& Wilbourne, P. L. (2002). Mesa Grande: A methodological analysis of clinical trials of treatment for alcohol use disorders. Addiction, 97(3), 265-277.

Molina BSG, Bukstein OG, Lynch KG. (2002). Attention-deficit/hyperactivity disorder and conduct disorder symptomatology in adolescents with alcohol use disorder. Psychology of Addictive Behaviors. 16 (2):161-164.

Morgenstern, J., Longabaugh, R. (2000). Cognitive-behavioral treatment for alcohol dependence: a review of evidence for its hypothesized mechanisms of action. Addiction, 95(10), 1475-1490.

Munro, C.A., Oswald, L.M., Weerts, E.M., McCaul, M.E., Wand, G.S. (2005). Hormone responses to social stress in abstinent alcohol-dependent subjects and social drinkers with no history of alcohol dependence. Alcohol Clin. Exp. Res., 29 (7): 1133-1138.

Myers, B., McKlveen, J.M., Herman, J.P. (2012). Neural regulation of the stress response: the many faces of feedback. Cell Mol Neurobiol. 32(5):683-694.

Nakovics, H., Diehl, A., Geiselhardt, H., Mann, K. (2009). Entwicklung und Validierung eines Instrumentes zur substanzunabhängigen Erfassung von Craving: Die Mannheimer Craving Scale (MaCS). Psychiatrische Praxis. 36: 7278.

Niemelä, O. (2016). Biomarker-Based Approaches for Assessing Alcohol Use Disorders. Int. J. Environ. Res. Public Health. 13 (2): 166.

O'Malley, S.S., Krishnan-Sarin, S., Farren, C., Sinha, R., Kreek, M.J. (2002).

Naltrexone decreases craving and alcohol self-administration in alcohol-dependent subjects and activates the hypothalamo-pituitary-adrenocortical axis.

Psychopharmacology. 160:19-29.

Oswald, L.M., Wong, D.F., McCaul, M., Zhou, Y., Kuwabara, H., Choi, L., et al. (2005). Relationships among ventral striatal dopamine release, cortisol secretion, and subjective responses to amphetamine. Neuropsychopharmacology. 30 (4): $821-$ 32. doi:10.1038/sj.npp.1300667.

Pape, H.-C., Kutz, A., Silbernagl, S. (2014) (Hrsg). Physiologie. 7. Auflage, Thieme Verlag.

Pettinati, H,. O'Brien, C., Dackis, C., et al. (2006). The status of naltrexone in the treatment of alcohol dependence: specific effects on heavy drinking. J Clin Psychopharmacol. 26: 610-625. 
Phelps, E.A. \& LeDoux, J.E. (2005). Contributions of the amygdala to emotion processing: from animal models to human behavior. Neuron 48: 175-187.

Pienaar, W.P., Roberts, M.C., Emsley, R.A. et al. (1995). The thyrotropin releasing hormone stimulation test in alcoholism. Alcohol and Alcoholism 30(5):661-667.

Presscot, C.A. \& Kendler, K.S. (1999). Genetic and environmental contributions to alcohol abuse and dependence in a population-based sample of male twins. Am. J. Psychiatry, 156 (1): 34-40.

Preuss, U.W., Schuckit, M.A., Smith, T.L., Danko, G.P., Bucholz, K.K., Hesselbrock, M.N. et al. (2003). Predictors and correlates of suicide attempts over 5 years in 1237 alcohol-dependent men and women. American Journal of Psychiatry. 160, 56-63.

Preuss, U.W., Koller, G., Barnow, S., Eikmeier, M., Soyka, M. (2006). Suicidal behavior in alcohol-dependent subjects: the role of personality disorders. Alcoholism: Clinc. Experim. Res. 30:866-877.

Preuss, U.W., Johann, M., Fehr, C., Koller, G., Wodarz, N., Hesselbrock, V., Wong, W.M., Soyka, M. (2009). Personality Disorders in Alcohol-Dependent Individuals: Relationship with Alcohol Dependence Severity. European Addiction Research, 15: 188-195.

Preuss, U.W., Ridiger, M., Rujescu, D., Samochowiec, J., Fehr, C., Wurst, F.M., Koller, G., Bondy, B., Wodarz, N., Debniak, T., Grzywacz, A., Soyka, M. \& Zill, P. (2011). Association of ADH4 genetic variants with alcohol dependence risk and related phenotypes: results from a larger multicenter association study. Add. Biol. 16 (2): 323-333.

Preuss, U.W., Gouzoulis-Mayfrank, E., Havemann-Reinecke, U., Schäfer, I., Beutel, M., Mann. K.F. \& Hoch, E. (2016). Psychische Komorbiditäten bei alkoholbedingten Störungen. Nervenarzt, 26 (1): 26-34.

Pruessner, J.C., Wolf, O.T., Hellhammer, D.H. et al. (1997). Free cortisol levels after awakening: a reliable biological marker for the assessment of adrenocortical activity. Life Sci. 61:2 539-2549.

Rachdaoui, N., Sarkar, D.K. (2013). Effects of alcohol on the endocrine system. Endocrinol Metab Clin North Am. 42 (3): 593-615.

Rangaswamy, M. \& Porjesz, B. (2014). Understanding alcohol use disorders with neuroelectrophysiology. Handb. Clin. Neurol., 125: 383-414.

Rehm, J., Gmel, G., Sempos, C., Trevisan, M. (2003). Alcohol-related mortality and morbidity. Alcohol Res Health. 27: 39-51.

Rehm, J., Imtiaz, S. A. (2016). A narrative review of alcohol consumption as a risk factor for global burden of disease. Subst Abuse Treat Prev Policy, 11: 37.

Rehm, J., Gmel, G.E., Gmel, G., et al. (2017). The relationship between different dimensions of alcohol use and the burden of disease - an update. Addiction. 112 (6): 968-1001.

Richardson, H.N., Lee, S.Y., O'Dell, L.E. et a., (2008). Alcohol selfadministration acutely stimulates the hypothalamic-pituitary-adrenal axis, but alcohol dependence leads to a dampened neuroendocrine state. European Journal of Neuroscience 28 (8):1641-1653.

Rist, F. (2002). Evidenzbasierte Therapie Alkoholabhängiger: Der Beitrag 
kognitiv-verhaltenstherapeutischer Verfahren. Sucht, 48 (3), 171-181.

Ritschel, M. \& Treutlein, J. (2013). The Genetics of alcohol dependence. Ann. N.Y. Acad. Sci. 1282: 39-70.

Roos, C.R., Maisto, S.A., Witkiewitz, K. (2017). Coping mediates the effects of cognitive-behavioral therapy for alcohol use disorder among outpatient clients in Project Match when dependence severity is high. Addiction, 112 (9): 1547-1557.

Roozendaal, B., McEwen, B. S., \& Chattarji, S. (2009). Stress, memory and the amygdala. Nature Reviews Neuroscience, 10 (6), 423-433.

Ruttle, P.L., Maslowsky, J., Armstrong, J.M., Burk, L.R., Essex, M.J. (2015). Longitudinal associations between diurnal cortisol slope and alcohol use across adolescence: A seven-year prospective study.

Psychoneuroendocrinology. 2015; 56: 23-28.

Ryan, R., Booth, S., Spathis, A., Mollart, S., Clow, A. (2016). Use of salivary diurnal cortisol as an outcome measure in randomised controlled trials: a systematic review. Ann Behav Med. 50(2):210-236.

Saal, D., Dong, Y., Bonci, A., Malenka, R.C. (2003). Drugs of abuse and stress trigger a common synaptic adaptation in dopamine neurons. Neuron. 37: 577-582.

Sapolsky, R.M., Romero, L.M., Munck, A.U. (2000). How do glucocorticoids influence stress responses? Integrating permissive, suppressive, stimulatory, and preparative actions. Endocrine Reviews. 2000;21 (1):55-89.

Saß, H., Wittchen, H.-U., Zaudig, M. (1996). DSM-IV. Göttingen: Hogrefe.

Sawyer, K.S., Poey, A., Ruiz, S.M., Marinkovic, K., Oscar-Berman, M. (2015). Measures of skin conductance and heart rate in alcoholic men and women during memory performance. PeerJ. 2015; 3: e941.

Sayette, M.A. (1999). Does drinking reduce stress? Alcohol Research and Health, 23: 250-255.

Schandry, R. (2016). Biologische Psychologie. 4., vollständig überarbeitete Auflage Beltz (Verlag) 978-3-621-28182-9 (ISBN).

Schaufeli, W.B. (2017). Burnout: A Short Socio-Cultural History (pp 105-127). In Neckel, S., Schaffner, A.K., Wagner, G. (Eds.) Burnout, Fatigue, Exhaustion. An inter-disciplinary Perspective on a Modern Affliction. Palgrave Macmillan.

Schäfer, I., Najavits, L.M. (2007). Clinical challenges in the treatment of patients with posttraumatic stress disorder and substance abuse. Current Opinion in Psychiatry. 20: 614-618.

Schäfer, I., Teske, L., Schulze-Thusing, J., et al. (2010). Impact of childhood trauma on hypothalamus-pituitary-adrenal axis activity in alcohol-dependent patients. European Addiction Research. 16 (2): 108-114.

Schäfer, I., Pawils, S., Driessen, M., Härter, M., Hillemacher, T., Klein, M., Muehlhan, M., Ravens-Sieberer, U., Schäfer , M., Scherbaum , N., Schneider, B., Thomasius, R., Wiedemann, K., Wegscheider, K., Barnow, S. (2017). Understanding the role of Childhood Abuse and Neglect as a cause and consequence of Substance Abuse: The German CANSAS-Network. European Journal of Psychotraumatology (EJPT. VOL. 8. 
Schepis, T.S., Rao, U., Yadav, H., Adinoff, B. (2011). The limbic-hypothalamicpituitary-adrenal axis and the development of alcohol use disorders in youth. Alcoholism: Clinical and Experimental Research, 35 (4): 595-605.

Schmidt, R.F. \& Schaible, H.G. (2005). Neuro- und Sinnesphysiologie. 5 Auflage, Springer Verlag, Berlin.

Schmidt, P., Kufner H., Hasemann, S., Loehnert, B., Kolb, W., Zemlin, U., Soyka, M. (2007). Ist der European Addiction Severity Index ein sinnvolles Diagnoseinstrument bei Alkoholabhängigkeit? Fortschr. Neurol. Psychiatrie, 75 (9): 541-548

Schmitt, M., Hübner, A., Maes, J. (2010). Validierung des Vereinfachten BeckDepressions-Inventars (BDI-V) an Fremdeinschätzungen. Diagnostica, 56, (3): 125132. Hogrefe Verlag.

Schneider, B., Georgi, K., Weber, B., Schnabel, A., Ackermann, H., Wetterling, T. (2006). Risk factors for suicide in substance-related disorders. Psychiatrische Praxis. 33, 81-87.

Schneider, B., Kölves, A., Blettner, M., Wetterling, T., Schnabel, A., Värnik, A. (2009). Substance use disorders as risk factors for suicide in an Eastern and a Central European city (Tallin and Frankfurt/Main). Psychiatry Research. 165, 263-272.

Schnell, T., Koethe, D., Daumann, J., Gouzoulis-Mayfrank, E. (2010). Prävalenz der Komorbidität Psychose und Sucht. Klinisch-epidemiologische Ergebnisse aus verschiedenen Behandlungssettings in einer deutschen Großstadt. Nervenarzt, 81, 323-328.

Schommer, N. C., Hellhammer, D. H., \& Kirschbaum, C. (2003). Dissociation between reactivity of the hypothalamus-pituitary-adrenal axis and the sympathetic-adrenal-medullary system to repeated psychosocial stress. Psychosomatic Medicine, 65(3), 450-460.

Schott, H. (2004). Das Alkoholproblem in der Medizingeschichte, In: Rainer Tölle und Elmar Doppelfeld (Hrsg.). Alkoholismus: Erkennen und Behandeln. Deutscher ÄrzteVerlag; 1. Auflage.

Schuckit, M.A., Smith, T.L. (1996). An 8-year follow-up of 450 sons of alcoholics and control subjects. Arch. Gen. Psychiatry, 53: 202-210.

Schuckit, M.A., Smith, T.L., Kalmijin, J., Tsuang, J., Hesselbrock, V., Buchholz, K. (2000). Response to alcohol in daughters of alcoholics: a pilot study and a comparison with sons of alcoholics. Alcohol, 35: 242-248.

Schuckit, M.A. (2006). Comorbidity between substance use disorders and psychiatric conditions. Addiction, 101 (Suppl 1): 76-88.

Schuckit, M.A. (2009). An overview of genetic influences in alcoholism. Journal of Substance Abuse Treatment, 36: 5-14.

Schuckit, M.A. (2014), A Brief History of Research on the Genetics of Alcohol and other Drug Use Disorders. J. Stud. Alcohol Drugs, Supplement 17, 59-67.

Seitz, H.K., Becker, P. (2007). Alcohol metabolism and cancer risk. Alcohol Res Health. 30(1):38-47. 
Seitz, H.K., Bühringer, G., Mann, K. (2008). Grenzwerte für den Konsum alkoholhaltiger Getränke. In: DHS (eds): Jahrbuch Sucht 2008, Geesthacht: Neuland Verlagsgesellschaft mbH 2009.

Selye, H. (1950). Stress and the General Adaptation Syndrome. B Med J. 17 (4667): 1383-1392.

Selye, H. (1998). A syndrome produced by diverse nocuous agents. J. Neuropsychiatry Clin. Neurosci. 10: 230-231. (In Nature, 1936, Vol. 138, Nr. 3479, S. 32).

Sher, K.J., Bartholow, B.D., Peuser, K., Erickson, D.J., Wood, M.D. (2007). Stress response dampening effects of alcohol: Attention as a mediator and moderator. Journal of Abnormal Psychology. 116: 362-377.

Siegrist J, Geyer S. (2002). ILE - Inventar zur Erfassung lebensverändernder Ereignisse; In: Brähler E, Schumacher J, Strauß B; (Hrsg.), Diagnostische Verfahren in der Psychotherapie; 2. Auflage; Hogrefe Verlag, Göttingen Bern Toronto Seattle. 211-213.

Simmons, D.A., Broderick, P.A. (2005). Cytokines, stressors, and clinical depression: augmented adaptation responses underlie depression pathogenesis. Prog. Neuropsychopharmacol. Biol. Psychiatry. 29 (5): 793-807.

Simpson, T. L., Miller, W. R. (2002). Concomitance between childhood sexual and physical abuse and substance use problems. A review. Clinical Psychology Review. 22 (1): 27-77.

Singer, M.V., Teyssen, S. (2005). Hrsg.: Alkohol und Alkoholfolgekrankheiten. Grundlagen - Diagnostik - Therapie. Berlin, Heidelberg, New York: Springer-Verlag.

Sinha, R. (2007). The role of stress in addiction relapse. Curr Psychiatry Rep. 9(5): 388-395.

Sinha, R. (2008). Chronic stress, drug use, and vulnerability to addiction. Ann NY Acad. Sci., 1141: 105-130.

Sinha, R., Fox, H.C., Hong, K.A., Bergquist, K.L., Bhagwager, Z., Siedlarz, K.M. (2009). Enhanced negative emotion and alcohol craving, and altered physiologic responses following stress and cue exposure in alcohol dependent individuals. Neuropsychopharmacology, 34 (5): 1198-1208.

Sinha, R. (2011). New Findings on Biological Factors Predicting Addiction Relapse Vulnerability. Curr. Psychiatry Rep.; 13 (5): 398-405.

Sinha, R. (2012). How Does Stress Lead to Risk of Alcohol Relapse? Alcohol. Res.; 34 (4): 432-440

Slutske, W.S., Piasecki, T.M., Hunt-Carter, E.E. (2003). Development and initial validation of the Hangover Symptoms Scale: prevalence and correlates of Hangover Symptoms in college students. Alcohol Clin Exp Res. 27:1442-1450.

Smith, T.L., Klein, M.H., Benjamin, L.S. (2003). Validation of the Wisconsin Personality Disorders Inventory with the SCID-II. Journal of Personality Disorders, 17 (3): 173-187.

Söderpalm Gordh, A.H., Söderpalm, B. (2011). Healthy subjects with a family history of alcoholism show increased stimulative subjective effects of alcohol. Alcoholism: 
Clinical and Experimental Research. 35: 1426-1434.

Soyka, M., Lieb, M. (2004). Depression und Alkoholabhängigkeit - Neue Befunde zur Komorbidität, Neurobiologie und Genetik. Journal für Neurologie, Neurochirurgie und Psychiatrie, 5 (3): 37-46.

Soyka, M., Preuss, U.W., Hesselbrock, V., Zill, P., Koller, G. \& Bondy, B. (2008). GABA-A2 receptor subunit gene (GABRA2) polymorphisms and risk for alcohol dependence. J. Psychiatr. Res. 42: 184-191.

Soyka, M., \& Küfner, H. (2008). Alkoholismus - Missbrauch und Abhängigkeit. 6. Auflage. Stuttgart: Thieme Verlag.

Spanagel, R. (2009). Alcoholism: a systems approach from molecular physiology to addictive behavior. Physiol. Rev. 89: 649-705.

Spielberger, C.D., Gorsuch, R.L., Lushene, R.E. (1970). Manual for the state trait inventory. Palo Alto, CA: Consulting Psychologists Press.

Starke, K., van Holst R., van den Brink W., Veltman D., Goudriaan A. (2013). Physiological and Endocrine Reactions to Psychosocial Stress in Alcohol Use Disorders: Duration of Abstinence Matters. Alcohol Clin. Exp. Res., 37 (8): 13431350.

Stephens, M.A., Wand, G. (2012). Stress and the HPA axis: role of glucocorticoids in alcohol dependence. Alcohol Res. 34 (4): 468-483.

Stephens, M.A.C., McCaul M.E., Wand, G.S. (2014). The Potential Role of Glucocorticoids and the HPA Axis in Alcohol Dependence. In Neurobiology of Alcohol Dependence 2014; Chapter 21, pp. 429-450. Elsevier Inc. DOI: 10.1016/ B978-012-405941-2.00021-3.

Steptoe, A., Hamer. M., Chida, Y. (2007). The effects of acute psychological stress on circulating inflammatory factors in humans: A review and meta-analysis. Brain Behav. Immun. 21: 901-912.

Sterling, P. \& Eyer, J. (1988). Allostasis: A New Paradigm to Explain Arousal Pathology. In Fischer S. \& Reason, J. (Hrsg.), Handbook of Life Stress, Cognition and Health. (S. 629-649). John Wiley \& Sons, New York.

Stockwell, T., Murphy, D., Hodgson, R. (1979). The Development of a Questionnaire to Measure Severity of Alcohol Dependence. British Journal of Addiction; 75: 79-87.

Sullivan, L.E., Fiellin, D.A. \& O'Connor, P.G. (2005). The prevalence and impact of alcohol problems in major depression: a systematic review. Am J.Med. 118 (4): 330341.

Sullivan, M.A, Rudnik-Levin F. (2001). Attention deficit/hyperactivity disorder and substance abuse. Diagnostic and therapeutic considerations. Ann N Y Acad Sci. 931: 251-270.

Tabakoff, B., Hoffman P. L. (2013). The neurobiology of alcohol consumption and alcoholism: an integrative history. Pharmacol. Biochem. Behav. 113, 20-37.

Thomas, S.E., Randall, P.K., Brady, K., See, R.E., Drobes, D.J. (2011). An acute psychosocial stressor does not potentiate alcohol cue reactivity in non-treatmentseeking alcoholics. Alcohol Clin. Exp. Res., 35 (3): 464-473. 
Thomas, S., Bacon, A.K., Sinha, R., Uhart, M., Adinoff, B. (2012). Clinical laboratory stressors uses to study alcohol-stress relationships. Alcohol Research: Current Reviews. 2012;34:459-467.

Tolic, I. \& Soyka, M. (2018). Stressreagibilität bei Alkoholabhängigen unter Berücksichtigung von Abstinenzdauer und Krankheitsschwere. Fortschr. Neurol. Psychiatr.; 86(06): 356-367. DOI: 10.1055/s-0043-119798.

Traue, H.C., Hrabal, V., Kosarz, P. (2000). Alltagsbelastungsfragebogen (ABF): Zur inneren Konsistenz, Validierung und Stressdiagnostik mit dem deutschsprachigen Daily Stress Inventory. Verhaltenstherapie \& Verhaltensmedizin, 21: 15-38.

True, W.R., Xian, H., Scherrer, J.F., Madden, P.A., Bucholz, K.K., Heath, A.C., Tsuang, M. (1999). Common genetic vulnerability for nicotine and alcohol dependence in men. Archives of general psychiatry 56, 655-661.

Tsigos, C., Kyrou, I., Kassi, E., \& Chrousos G. P. Last Update March 10, 2016. Stress, endocrine physiology and pathophysiology. In De Groot L.J., Chrousos G., Dungan K., Feingold K.R., Grossman A., Hershman J.M., Koch C., Korbonits M., McLachlan R., New M., Purnell J., Rebar R., Singer F., Vinik A. (Eds.) 2000. Last Update: March 10, 2016.

Uhart M, Oswald L, McCaul ME, Chang R, Wand GS. (2006). Hormonal responses to psychological stress and family history for alcoholism. Neuropsychopharmacology. 31:2255-2263.

Uhart, M. \& Wand, G.S. (2009). Stress, alcohol and drug interaction: an update of human research. Addict Biol. 14 (1): 43-64.

Ulrich-Lai, Y. M., \& Herman, J. P. (2009). Neural regulation of endocrine and autonomic stress responses. Nature Reviews Neuroscience, 10 (6), 397-409.

Veenstra, M.Y., Lemmens, P.H., Friesema, I.H., Garretsen, H.F., Knottnerus, J.A., Zwietering, P.J. (2006). A literature overview of the relationship between life-events and alcohol use in the general population. Alcohol \& Alcoholism, 41 (4): 455-463.

Velasco, M., Gomez, J., Blanco, M., Rodriguez, I. (1997). The cold pressor test: Pharmacological and therapeutic aspects. American Journal of Therapeutics. 4 (1):34-38.

Veltrup, C. (2002). Motivation. In J. Fengler (Hrsg.), Handbuch der Suchtbehandlung. Beratung-Therapie-Prävention. (S. 362-366). Landsberg: Ecomed.

Verster, J.C., Stephens, R., Penning, R., Rohsenow, D.J., McGeary, J., Levy, D., et al., on behalf of the Alcohol Hangover Research Group (2010). The Alcohol Hangover Research Group consensus statement on best practice in alcohol hangover research. Current Drug Abuse Reviews. 3: 116-127.

Volkow, N.D., Fowler, J.S., Wang, G.J. (2003). The addicted human brain: insights from imaging studies. J Clin Invest. 111:1444-1451.

Walter, M., Gerhard, U., Gerlach, M., Weijers, H-G., Böning, J., Wiesbeck, G.A. (2006). Cortisol concentrations, stress-coping styles after withdrawal and long-term abstinence in alcohol dependence. Addiction Biology, 11: 157-162. 
Wand, G.S., Dobs, A.S. (1991). Alterations in the hypothalamic-pituitary-adrenal axis in actively drinking alcoholics. Journal of Clinical Endocrinology and Metabolism 72(6):1290-1295.

Wille N., Bettge, S., Ravens-Sieberer, U., and the BELLA study group. (2008). Risk and protective factors for children's and adolescents' mental health: results of the BELLA study. European Child \& Adolescent Psychiatry, Vol. 17, Supplement 1 (2008).

Wilens TE, Kwon A, Tanguay S, Chase R, Moore H, Faraone SV, et al. (2005). Characteristics of adults with attention deficit hyperactivity disorder plus substance usedisorder: the role of psychiatric comorbidity. Am J Addict. 14:319-327.

Wilcox, H.C., Conner, K.R., Caine, E.D. (2004). Association of alcohol and drug use disorders and completed suicide: an empirical review of cohort studies. Drug Alcohol Depend. 76 (Suppl): S. 11-19.

Wittchen, H-U., Zaudig, M., Fydrich, T. (1997). Strukturiertes Klinisches Interview für DSM-IV. Achse I: Psychische Störungen / Achse II: Persönlichkeitsstörungen (SKIDII Persönlichkeitsstörungen). Göttingen, Hogrefe Verlag.

Wüst, S., Federenko, I. S., van Rossum, E. F. C., Koper, J. W., Kumsta, R., Entringer, S., Hellhammer, D.H. (2004). A psychobiological perspective on genetic determinants of hypothalamus-pituitary-adrenal axis activity. Annals of the New York Academy of Sciences, 1032, 52-62.

Yaldizli, O., Kuhl, H.C., Graf, M., Wiesbeck, G.A., Wurst, F.M. (2010). Risk factors for suicide attempts in patients with alcohol dependence or abuse and a history of depressive symptoms: a subgroup analysis from the WHO/ISBRA study. Drug and Alcohol Review, 29, 64-74.

Yoon, G., Kim, S.W., Thuras, P., Grant, J.E., Westermeyer, J. (2006). Alcohol craving in outpatients with alcohol dependence: rate and clinical correlates. J. Stud. Alcohol. 67 (5): 770-777.

Young, E. A., Abelson, J., Lightman, S. L. (2004). Cortisol pulsatility and its role in stress regulation and health. Front. Neuroendocrinol. 25, 69-76.

Zanarini, M.C., Frankenburg, F.R., Hennen, J., Reich, D.B., Silk, K.R. (2004). Axis I comorbidity in patients with borderline personality disorder: 6-year follow-up and prediction of time to remission. Am. J. Psychiatry 161: 2108-2114.

Zikos, E., Gill, K.J., Charney, D.A. (2010). Personality disorders among alcoholic outpatients: prevalence and course in treatment. Can. J. Psychiatry, 55: 65-73.

Zimmermann, U., Spring, K., Kunz-Ebrecht, S.R,, Uhr, M., Wittchen, H-U., Holsboer F. (2004). Effect of ethanol on hypothalamic-pituitary-adrenal system response to psychosocial stress in sons of alcohol-dependent

fathers. Neuropsychopharmacology. 29:1156-1165. 

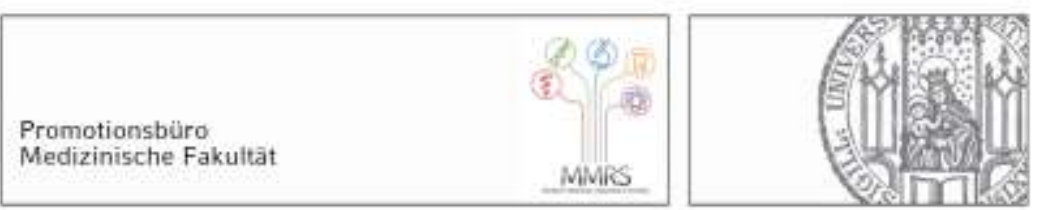

Eidesstattliche Versicherung

\section{Tolic Ilija}

Name, Vorname

Ich erkläre hiermit an Eides statt,

dass ich die vorliegende Dissertation mit dem Titel

Stressreagibilităt bei Alkoholabhăngigen unter Berücksichtigung von Abstinenzdauer und Krankheitsschwere

selbständig verfasst, mich außer der angegebenen keiner weiteren Hilfsmittel bedient und alle Erkenntnisse, die aus dem Schrifttum ganz oder annähernd übernommen sind, als solche kenntlich gemacht und nach ihrer Herkunft unter Bezeichnung der Fundstelle einzeln nachgewiesen habe.

Ich erkläre des Weiteren, dass die hier vorgelegte Dissertation nicht in gleicher oder in ähnlicher Form bei einer anderen Stelle zur Erlangung eines akademischen Grades eingereicht wurde.

München, 27.04.2020

Ort, Datum
Ilija Tolic

Unterschrift Doktorandin bzw. Doktorand 


\section{Anhang}

Diagramm 1. Ausprägung depressiver Symptome (BDI) in allen Stichproben. Stichprobe Alkoholabhängiger aufgeteilt nach Abstinenzdauer und Krankheitsschwere (aus Tolic \& Soyka, 2018).

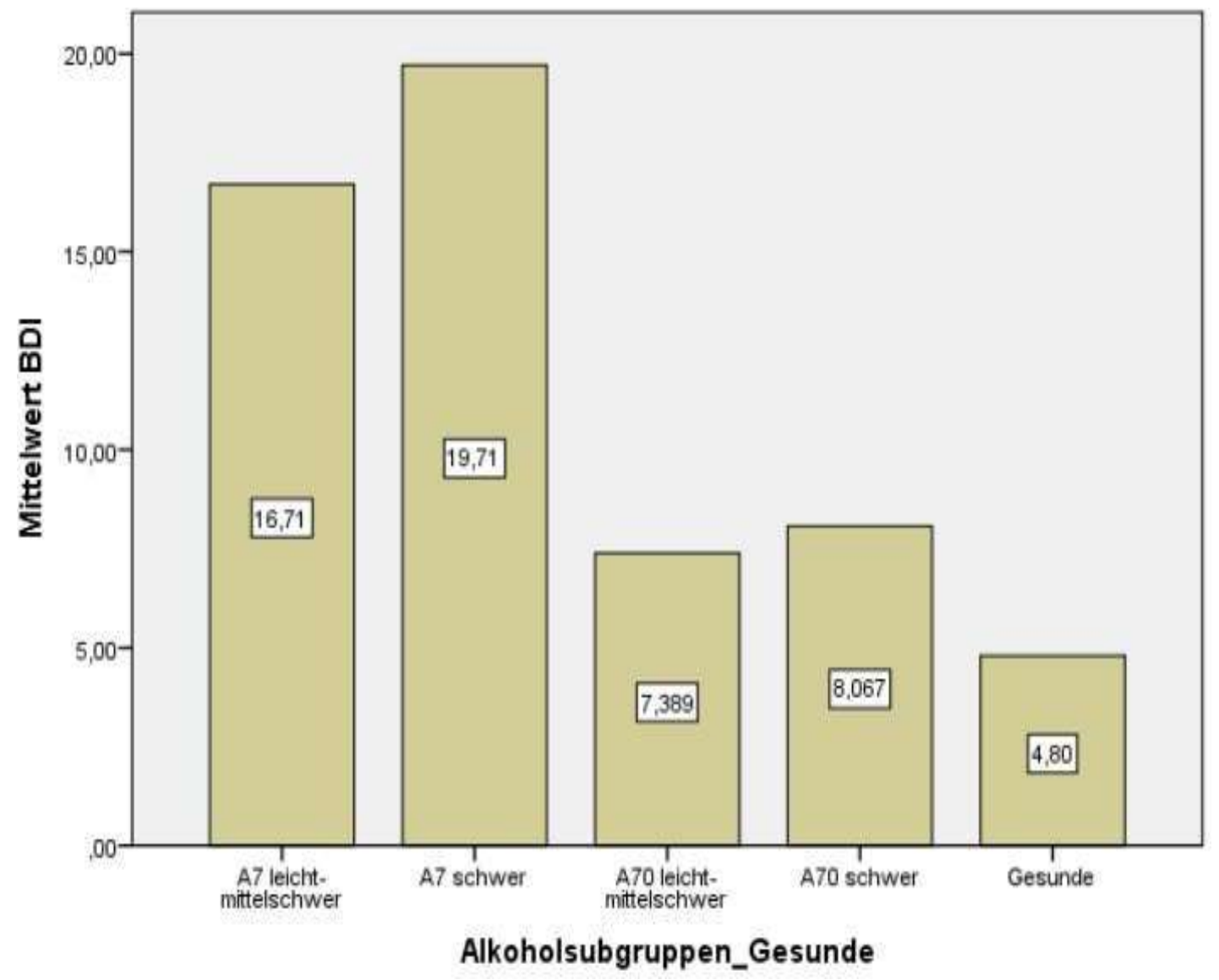


Tabelle 2. Cortisol: Mehrfachvergleiche der Versuchsgruppen. Alkoholabhängige aufgeteilt nach Schweregrad (aus Tolic \& Soyka, 2018).

\begin{tabular}{|c|c|c|c|c|c|c|c|}
\hline \multirow[b]{2}{*}{$\begin{array}{l}\text { Abhängige } \\
\text { Variable }\end{array}$} & \multirow[b]{2}{*}{$\begin{array}{l}\text { (I) } \\
\text { Versuchsgruppen }\end{array}$} & \multirow[b]{2}{*}{$\begin{array}{l}\text { (J) } \\
\text { Versuchsgruppen }\end{array}$} & \multirow{2}{*}{$\begin{array}{l}\text { Mittelwert- } \\
\text { differenz (I- } \\
\text { J) }\end{array}$} & \multirow{2}{*}{$\begin{array}{l}\text { Stan- } \\
\text { dard- } \\
\text { fehler }\end{array}$} & \multirow[b]{2}{*}{ Sig. } & \multicolumn{2}{|c|}{$95 \%$ Konfidenzintervall } \\
\hline & & & & & & Untergrenze & Obergrenze \\
\hline \multirow[t]{6}{*}{ CORTISOL(T0) } & Leicht- & Schwer & $-1,46404^{*}$ & ,54960 & ,033 & $-2,8318$ &,- 0963 \\
\hline & mittelschwer & Gesund & $1,68619^{*}$ &, 54457 &, 010 & ,3310 & 3,0414 \\
\hline & Schwer & Leicht-mittelschwer & $1,46404^{*}$ & ,54960 & ,033 & ,0963 & 2,8318 \\
\hline & & Gesund & $3,15023^{*}$ & ,56998 &, 000 & 1,7318 & 4,5687 \\
\hline & Gesund & Leicht-mittelschwer & $-1,68619^{*}$ & ,54457 & 010 & $-3,0414$ &,- 3310 \\
\hline & & Schwer & $-3,15023^{*}$ & ,56998 & ,000 & $-4,5687$ & $-1,7318$ \\
\hline \multirow[t]{6}{*}{ CORTISOL(T1) } & Leicht- & Schwer & $-1,50374^{*}$ & ,56326 & ,032 & $-2,9055$ &,- 1020 \\
\hline & mittelschwer & Gesund & $1,75810^{*}$ & ,55811 & ,009 & ,3692 & 3,1470 \\
\hline & Schwer & Leicht-mittelschwer & $1,50374^{*}$ & ,56326 & ,032 & , 1020 & 2,9055 \\
\hline & & Gesund & $3,26184^{*}$ & ,58414 &, 000 & 1,8081 & 4,7155 \\
\hline & Gesund & Leicht-mittelschwer & $-1,75810^{*}$ & ,55811 &, 009 & $-3,1470$ &,- 3692 \\
\hline & & Schwer & $-3,26184^{*}$ &, 58414 & ,000 & $-4,7155$ & $-1,8081$ \\
\hline \multirow[t]{6}{*}{ CORTISOL(T2) } & Leicht- & Schwer & $-1,68384$ & ,77645 & , 101 & $-3,6161$ & ,2484 \\
\hline & mittelschwer & Gesund & $-2,37810^{*}$ & ,76934 & 011 & $-4,2927$ &,- 4635 \\
\hline & Schwer & Leicht-mittelschwer & 1,68384 & ,77645 & ,101 &,- 2484 & 3,6161 \\
\hline & & Gesund &,- 69425 & ,80523 & 691 & $-2,6981$ & 1,3096 \\
\hline & Gesund & Leicht-mittelschwer & $2,37810^{*}$ & ,76934 & 011 &, 4635 & 4,2927 \\
\hline & & Schwer & ,69425 & ,80523 & 691 & $-1,3096$ & 2,6981 \\
\hline \multirow[t]{6}{*}{ CORTISOL(T3) } & Leicht- & Schwer & $-1,88946^{*}$ & ,75015 & ,047 & $-3,7563$ &,- 0226 \\
\hline & mittelschwer & Gesund & ,98571 & ,74328 & ,419 &,- 8640 & 2,8354 \\
\hline & Schwer & Leicht-mittelschwer & $1,88946^{\star}$ & ,75015 & ,047 & ,0226 & 3,7563 \\
\hline & & Gesund & $2,87517^{*}$ & ,77796 & ,002 & ,9392 & 4,8112 \\
\hline & Gesund & Leicht-mittelschwer &,- 98571 & ,74328 & 419 & $-2,8354$ & 8640 \\
\hline & & Schwer & $-2,87517^{*}$ & ,77796 & ,002 & $-4,8112$ &,- 9392 \\
\hline \multirow[t]{6}{*}{ CORTISOL(T4) } & Leicht- & Schwer & $-1,88010^{*}$ & ,59631 & ,009 & $-3,3641$ &,- 3961 \\
\hline & mittelschwer & Gesund & $1,62714^{*}$ & ,59085 & ,026 &, 1567 & 3,0975 \\
\hline & Schwer & Leicht-mittelschwer & $1,88010^{*}$ & ,59631 & ,009 & ,3961 & 3,3641 \\
\hline & & Gesund & $3,50724^{*}$ & ,61842 &, 000 & 1,9682 & 5,0462 \\
\hline & Gesund & Leicht-mittelschwer & $-1,62714^{*}$ & ,59085 &, 026 & $-3,0975$ &,- 1567 \\
\hline & & Schwer & $-3,50724^{*}$ & ,61842 &, 000 & $-5,0462$ & $-1,9682$ \\
\hline \multirow[t]{6}{*}{ CORTISOL(T5) } & Leicht- & Schwer & $-2,40936^{\star}$ & ,52611 & 000 & $-3,7186$ & $-1,1001$ \\
\hline & mittelschwer & Gesund & $1,36190^{*}$ &, 52129 & ,037 &, 0646 & 2,6592 \\
\hline & Schwer & Leicht-mittelschwer & $2,40936^{*}$ & ,52611 & ,000 & 1,1001 & 3,7186 \\
\hline & & Gesund & $3,77126^{*}$ &, 54561 &, 000 & 2,4135 & 5,1291 \\
\hline & Gesund & Leicht-mittelschwer & $-1,36190^{*}$ & ,52129 & ,037 & $-2,6592$ &,- 0646 \\
\hline & & Schwer & $-3,77126^{*}$ &, 54561 &, 000 & $-5,1291$ & $-2,4135$ \\
\hline
\end{tabular}

\footnotetext{
*. die Mittelwertdifferenz ist auf der Stufe 0.05 signifikant.
} 
Tabelle 3. Cortisol: Auswertung nach Schweregrad und Abstinenzdauer (aus Tolic \& Soyka, 2018).

\begin{tabular}{|c|c|c|c|c|c|c|}
\hline $\begin{array}{ll}\text { Abhängige } & \text { Versuchsgruppe }(\mathrm{I}) \\
\text { Variable } & \text { Versuchsgruppe }(\mathrm{J})\end{array}$ & $\mathrm{N}$ & $M$ & SD & Df & $F$ & Sig. \\
\hline $\begin{array}{l}\text { Cortisol (T0) A7-Leicht-mittelschwer } \\
\text { A7-Schwer } \\
\text { A70-Leicht-mittelschwer } \\
\text { A70-Schwer } \\
\text { Gesund }\end{array}$ & $\begin{array}{l}17 \\
14 \\
18 \\
15 \\
30\end{array}$ & $\begin{array}{l}4,88 \\
7,50 \\
3,83 \\
4,22 \\
2,65\end{array}$ & $\begin{array}{l}2,45 \\
3,03 \\
1,94 \\
1,44 \\
1,15 \\
\end{array}$ & 4,89 & 14.928 & .00 \\
\hline $\begin{array}{l}\text { Cortisol (T1) A7-Leicht-mittelschwer } \\
\text { A7-Schwer } \\
\text { A70-Leicht-mittelschwer } \\
\text { A70-Schwer } \\
\text { Gesund }\end{array}$ & $\begin{array}{l}17 \\
14 \\
18 \\
15 \\
30\end{array}$ & $\begin{array}{l}4,92 \\
7,54 \\
4,00 \\
4,47 \\
2,69\end{array}$ & $\begin{array}{l}2,49 \\
3,01 \\
2,20 \\
1,66 \\
1,21\end{array}$ & 4,89 & 13.562 & .00 \\
\hline $\begin{array}{l}\text { Cortisol (T2) A7-Leicht-mittelschwer } \\
\text { A7-Schwer } \\
\text { A70-Leicht-mittelschwer } \\
\text { A70-Schwer } \\
\text { Gesund }\end{array}$ & $\begin{array}{l}17 \\
14 \\
18 \\
15 \\
30\end{array}$ & $\begin{array}{l}6,75 \\
9,73 \\
7,21 \\
7,68 \\
9,36\end{array}$ & $\begin{array}{l}3,53 \\
3,09 \\
3,38 \\
2,68 \\
2,73\end{array}$ & 4,89 & 3.471 & .01 \\
\hline $\begin{array}{l}\text { Cortisol (T3) A7-Leicht-mittelschwer } \\
\text { A7-Schwer } \\
\text { A70-Leicht-mittelschwer } \\
\text { A70-Schwer } \\
\text { Gesund }\end{array}$ & $\begin{array}{l}17 \\
14 \\
18 \\
15 \\
30\end{array}$ & $\begin{array}{c}7,70 \\
10,72 \\
8,60 \\
9,42 \\
7,18\end{array}$ & $\begin{array}{l}3,57 \\
3,35 \\
3,46 \\
3,27 \\
1,74\end{array}$ & 4,89 & 4.074 & .00 \\
\hline $\begin{array}{l}\text { Cortisol (T4) A7-Leicht-mittelschwer } \\
\text { A7-Schwer } \\
\text { A70-Leicht-mittelschwer } \\
\text { A70-Schwer } \\
\text { Gesund }\end{array}$ & $\begin{array}{l}17 \\
14 \\
18 \\
15 \\
30\end{array}$ & $\begin{array}{c}7,08 \\
10,20 \\
7,38 \\
8,10 \\
5,61\end{array}$ & $\begin{array}{l}2,57 \\
3,12 \\
2,72 \\
1,98 \\
1,51\end{array}$ & 4,89 & 9.911 & .00 \\
\hline $\begin{array}{l}\text { Cortisol (T5) A7-Leicht-mittelschwer } \\
\text { A7-Schwer } \\
\text { A70-Leicht-mittelschwer } \\
\text { A70-Schwer } \\
\text { Gesund }\end{array}$ & $\begin{array}{l}17 \\
14 \\
18 \\
15 \\
30\end{array}$ & $\begin{array}{l}6,36 \\
9,09 \\
4,54 \\
6,66 \\
4,06\end{array}$ & $\begin{array}{l}2,43 \\
3,17 \\
1,50 \\
1,06 \\
1,28\end{array}$ & 4,89 & 19.451 & .00 \\
\hline
\end{tabular}


Tabelle 4b. EDA: Varianzhomogenitätstest und die Varianzanalyse (ANOVA)

\section{Varianzhomogenitätstest}

\begin{tabular}{|c|c|c|c|c|}
\hline & Levene-Statistik & df1 & $\mathrm{df} 2$ & Sig. \\
\hline EDA TSST Vorbereitung & 248 & 2 & 91 & ,781 \\
\hline EDA TSST Rede & 4,561 & 2 & 91 & ,013 \\
\hline EDA TSST Kopfrechnen & 1,023 & 2 & 91 & ,364 \\
\hline EDA TSST Ruhe & ,395 & 2 & 91 & ,675 \\
\hline EDA TSST Ende & ,712 & 2 & 91 & 493 \\
\hline
\end{tabular}

ANOVA

\begin{tabular}{|c|c|c|c|c|c|c|}
\hline & & Quadratsumme & Df & $\begin{array}{l}\text { Mittel der } \\
\text { Quadrate }\end{array}$ & $\mathrm{F}$ & Sig. \\
\hline $\begin{array}{l}\text { EDA TSST } \\
\text { Vorbereitung }\end{array}$ & $\begin{array}{l}\text { Zwischen Gruppen } \\
\text { Innerhalb der Gruppen } \\
\text { Gesamtsumme }\end{array}$ & $\begin{array}{r}8,570 \\
97,172 \\
105,742\end{array}$ & $\begin{array}{r}2 \\
91 \\
93\end{array}$ & $\begin{array}{l}4,285 \\
1,068\end{array}$ & 4,013 & ,021 \\
\hline $\begin{array}{l}\text { EDA TSST } \\
\text { Rede }\end{array}$ & $\begin{array}{l}\text { Zwischen Gruppen } \\
\text { Innerhalb der Gruppen } \\
\text { Gesamtsumme }\end{array}$ & $\begin{array}{r}4,190 \\
644,495 \\
648,685\end{array}$ & $\begin{array}{r}2 \\
91 \\
93\end{array}$ & $\begin{array}{l}2,095 \\
7,082\end{array}$ & ,296 & ,745 \\
\hline $\begin{array}{l}\text { EDA TSST } \\
\text { Kopfrechnen }\end{array}$ & $\begin{array}{l}\text { Zwischen Gruppen } \\
\text { Innerhalb der Gruppen } \\
\text { Gesamtsumme }\end{array}$ & $\begin{array}{r}20,871 \\
660,873 \\
681,744\end{array}$ & $\begin{array}{r}2 \\
91 \\
93\end{array}$ & $\begin{array}{r}10,436 \\
7,262\end{array}$ & 1,437 & 243 \\
\hline $\begin{array}{l}\text { EDA TSST } \\
\text { Ruhe }\end{array}$ & $\begin{array}{l}\text { Zwischen Gruppen } \\
\text { Innerhalb der Gruppen } \\
\text { Gesamtsumme }\end{array}$ & $\begin{array}{r}32,555 \\
158,221 \\
190,776\end{array}$ & $\begin{array}{r}2 \\
91 \\
93\end{array}$ & $\begin{array}{r}16,278 \\
1,739\end{array}$ & 9,362 &, 000 \\
\hline $\begin{array}{l}\text { EDA TSST } \\
\text { Ende }\end{array}$ & $\begin{array}{l}\text { Zwischen Gruppen } \\
\text { Innerhalb der Gruppen } \\
\text { Gesamtsumme }\end{array}$ & $\begin{array}{r}35,128 \\
116,149 \\
151,277\end{array}$ & $\begin{array}{r}2 \\
91 \\
93\end{array}$ & $\begin{array}{r}17,564 \\
1,276\end{array}$ & 13,761 &, 000 \\
\hline
\end{tabular}


Tabelle 5. Puls: Auswertung nach Schweregrad. (aus Tolic \& Soyka, 2018).

Scheffé

\begin{tabular}{|c|c|c|c|c|c|c|c|}
\hline \multirow{2}{*}{$\begin{array}{l}\text { Abhängige } \\
\text { Variable }\end{array}$} & \multirow{2}{*}{$\begin{array}{l}\text { (I) } \\
\text { Versuchsgrup- } \\
\text { pen }\end{array}$} & \multirow[b]{2}{*}{ (J) Versuchsgruppen } & \multirow{2}{*}{$\begin{array}{l}\text { Mittelwert } \\
\text {-differenz } \\
(\mathrm{I}-\mathrm{J})\end{array}$} & \multirow[b]{2}{*}{$\begin{array}{c}\text { Standard- } \\
\text { fehler }\end{array}$} & \multirow[b]{2}{*}{ Sig. } & \multicolumn{2}{|c|}{$\begin{array}{c}95 \% \\
\text { Konfidenzintervall }\end{array}$} \\
\hline & & & & & & $\begin{array}{c}\text { Untergren } \\
\text { ze }\end{array}$ & $\begin{array}{c}\text { Obergre } \\
\text { nze }\end{array}$ \\
\hline \multirow{6}{*}{$\begin{array}{l}\text { PULS } \\
\text { TSST- } \\
\text { Vorbereit }\end{array}$} & Leicht- & Schwer & $-3,92118$ & 1,83685 & , 108 & $-8,4923$ &, 6500 \\
\hline & mittelschwer & Gesund & 1,75238 & 1,82003 & ,631 & $-2,7769$ & 6,2817 \\
\hline & Schwer & Leicht-mittelschwer & 3,92118 & 1,83685 & ,108 &,- 6500 & 8,4923 \\
\hline & & Gesund & $5,67356^{*}$ & 1,90494 & ,015 & ,9329 & 10,4142 \\
\hline & Gesund & Leicht-mittelschwer & $-1,75238$ & 1,82003 & ,631 & $-6,2817$ & 2,7769 \\
\hline & & Schwer & $-5,67356^{*}$ & 1,90494 & ,015 & $-10,4142$ &,- 9329 \\
\hline \multirow{6}{*}{$\begin{array}{l}\text { PULS } \\
\text { TSST- } \\
\text { Rede }\end{array}$} & Leicht- & Schwer & $-9,26404$ & 4,44336 &, 120 & $-20,3218$ & 1,7937 \\
\hline & mittelschwer & Gesund &,- 45714 & 4,40268 & ,995 & $-11,4136$ & 10,4993 \\
\hline & Schwer & Leicht-mittelschwer & 9,26404 & 4,44336 & ,120 & $-1,7937$ & 20,3218 \\
\hline & & Gesund & 8,80690 & 4,60809 & ,167 & $-2,6608$ & 20,2746 \\
\hline & Gesund & Leicht-mittelschwer & ,45714 & 4,40268 & ,995 & $-10,4993$ & 11,4136 \\
\hline & & Schwer & $-8,80690$ & 4,60809 & ,167 & $-20,2746$ & 2,6608 \\
\hline \multirow{6}{*}{$\begin{array}{l}\text { PULS } \\
\text { TSST- } \\
\text { Kopfrechn }\end{array}$} & Leicht- & Schwer & $-6,39212$ & 3,69496 & ,229 & $-15,5874$ & 2,8031 \\
\hline & mittelschwer & Gesund & 2,89524 & 3,66113 & ,732 & $-6,2158$ & 12,0063 \\
\hline & Schwer & Leicht-mittelschwer & 6,39212 & 3,69496 & ,229 & $-2,8031$ & 15,5874 \\
\hline & & Gesund & 9,28736 & 3,83194 & ,058 &,- 2488 & 18,8235 \\
\hline & Gesund & Leicht-mittelschwer & $-2,89524$ & 3,66113 & ,732 & $-12,0063$ & 6,2158 \\
\hline & & Schwer & $-9,28736$ & 3,83194 & ,058 & $-18,8235$ & ,2488 \\
\hline \multirow{6}{*}{$\begin{array}{l}\text { PULS } \\
\text { TSST- } \\
\text { Ruhe }\end{array}$} & Leicht- & Schwer & $-3,59507$ & 1,44664 &, 050 & $-7,1952$ &, 0050 \\
\hline & mittelschwer & Gesund & $3,70952^{*}$ & 1,43340 &, 040 &, 1424 & 7,2767 \\
\hline & Schwer & Leicht-mittelschwer & 3,59507 & 1,44664 & ,050 &,- 0050 & 7,1952 \\
\hline & & Gesund & $7,30460^{*}$ & 1,50027 & ,000 & 3,5710 & 11,0382 \\
\hline & Gesund & Leicht-mittelschwer & $-3,70952^{*}$ & 1,43340 & ,040 & $-7,2767$ &,- 1424 \\
\hline & & Schwer & $-7,30460 *$ & 1,50027 &, 000 & $-11,0382$ & $-3,5710$ \\
\hline \multirow{6}{*}{$\begin{array}{l}\text { PULS } \\
\text { TSST- } \\
\text { Ende }\end{array}$} & Leicht- & Schwer & $-3,41281^{*}$ & 1,33127 &, 042 & $-6,7258$ &,- 0998 \\
\hline & mittelschwer & Gesund & $3,36190^{*}$ & 1,31908 &, 043 &, 0793 & 6,6446 \\
\hline & Schwer & Leicht-mittelschwer & $3,41281^{*}$ & 1,33127 &, 042 & ,0998 & 6,7258 \\
\hline & & Gesund & $6,77471^{*}$ & 1,38062 &, 000 & 3,3389 & 10,2105 \\
\hline & Gesund & Leicht-mittelschwer & $-3,36190^{\star}$ & 1,31908 & ,043 & $-6,6446$ &,- 0793 \\
\hline & & Schwer & $-6,77471^{*}$ & 1,38062 &, 000 & $-10,2105$ & $-3,3389$ \\
\hline
\end{tabular}

*. die Mittelwertdifferenz ist auf der Stufe 0.05 signifikant. 
Tabelle 6. Stressverarbeitungsfragebogen 120 (SVF 120): Aufteilung nach Schweregrad. Mehrfachvergleiche hinsichtlich Positiv- und Negativ-Strategien (aus Tolic \& Soyka, 2018).

Scheffé

\begin{tabular}{|c|c|c|c|c|c|c|c|}
\hline \multirow[b]{2}{*}{$\begin{array}{l}\text { Abhängig } \\
\text { e Variable }\end{array}$} & \multirow{2}{*}{$\begin{array}{l}\text { (I) } \\
\text { Versuchsgrup- } \\
\text { pen }\end{array}$} & \multirow[b]{2}{*}{ (J) Versuchsgruppen } & \multirow[b]{2}{*}{$\begin{array}{c}\text { Mittelwertdiffer- } \\
\text { enz (I-J) }\end{array}$} & \multirow[b]{2}{*}{$\begin{array}{r}\text { Standard- } \\
\text { fehler }\end{array}$} & \multirow[b]{2}{*}{ Sig. } & \multicolumn{2}{|c|}{$\begin{array}{c}95 \% \\
\text { Konfidenzintervall }\end{array}$} \\
\hline & & & & & & Untergrenze & Obergrenze \\
\hline \multirow{6}{*}{$\begin{array}{l}\text { SVF } \\
\text { Positive } \\
\text { Strategien }\end{array}$} & \multirow{2}{*}{$\begin{array}{l}\text { Leicht- } \\
\text { mittelschwer }\end{array}$} & Schwer & 3,46305 & 1,44345 &, 061 &,- 1291 & 7,0552 \\
\hline & & Gesund & $-8,43810^{*}$ & 1,43024 &, 000 & $-11,9974$ & $-4,8788$ \\
\hline & \multirow[t]{2}{*}{ Schwer } & Leicht-mittelschwer & $-3,46305$ & 1,44345 &, 061 & $-7,0552$ & ,1291 \\
\hline & & Gesund & $-11,90115^{\star}$ & 1,49697 &, 000 & $-15,6265$ & $-8,1758$ \\
\hline & \multirow[t]{2}{*}{ Gesund } & Leicht-mittelschwer & $8,43810^{*}$ & 1,43024 & ,000 & 4,8788 & 11,9974 \\
\hline & & Schwer & $11,90115^{\star}$ & 1,49697 &, 000 & 8,1758 & 15,6265 \\
\hline \multirow{6}{*}{$\begin{array}{l}\text { SVF } \\
\text { Negative } \\
\text { Strategien }\end{array}$} & \multirow{2}{*}{$\begin{array}{l}\text { Leicht- } \\
\text { mittelschwer }\end{array}$} & Schwer & $-4,50246^{*}$ & 1,60383 & ,023 & $-8,4937$ &,- 5112 \\
\hline & & Gesund & $10,49524^{*}$ & 1,58914 &, 000 & 6,5405 & 14,4500 \\
\hline & \multirow[t]{2}{*}{ Schwer } & Leicht-mittelschwer & $4,50246^{*}$ & 1,60383 & ,023 &, 5112 & 8,4937 \\
\hline & & Gesund & $14,99770^{*}$ & 1,66329 &, 000 & 10,8585 & 19,1369 \\
\hline & \multirow[t]{2}{*}{ Gesund } & Leicht-mittelschwer & $-10,49524^{*}$ & 1,58914 &, 000 & $-14,4500$ & $-6,5405$ \\
\hline & & Schwer & $-14,99770^{*}$ & 1,66329 &, 000 & $-19,1369$ & $-10,8585$ \\
\hline
\end{tabular}

${ }^{*}$. die Mittelwertdifferenz ist auf der Stufe 0.05 signifikant. 
Tabelle 7. Trierer Inventar zum chronischen Stress (TICS). Aufteilung nach Schweregrad (aus Tolic \& Soyka, 2018).

Scheffé

\begin{tabular}{|c|c|c|c|c|c|c|c|}
\hline \multirow[b]{2}{*}{$\begin{array}{l}\text { Abhängige } \\
\text { Variable }\end{array}$} & \multirow[b]{2}{*}{ (I) Versuchsgruppen } & \multirow[b]{2}{*}{ (J) Versuchsgruppen } & \multirow[b]{2}{*}{ Mittelwertdifferenz (I-J) } & \multirow[b]{2}{*}{$\begin{array}{l}\text { Standard- } \\
\text { fehler }\end{array}$} & \multirow[b]{2}{*}{ Sig. } & \multicolumn{2}{|c|}{$\begin{array}{c}95 \% \\
\text { Konfidenzintervall }\end{array}$} \\
\hline & & & & & & $\begin{array}{l}\text { Unter- } \\
\text { grenze }\end{array}$ & $\begin{array}{l}\text { Ober- } \\
\text { grenze }\end{array}$ \\
\hline \multirow[t]{5}{*}{$\begin{array}{l}\text { TICS- } \\
\text { Uebe }\end{array}$} & Leicht-mittelschwer & $\begin{array}{l}\text { Schwer } \\
\text { Gesund }\end{array}$ & $\begin{array}{r}-3,06700^{*} \\
1,22381\end{array}$ & $\begin{array}{l}1,16449 \\
1,15383\end{array}$ & $\begin{array}{l}, 035 \\
, 572\end{array}$ & $\begin{array}{l}-5,9649 \\
-1,6476\end{array}$ & $\begin{array}{l}-, 1690 \\
4,0952\end{array}$ \\
\hline & Schwer & Leicht-mittelschwer & $3,06700^{*}$ & 1,16449 &, 035 & , 1690 & 5,9649 \\
\hline & & Gesund & $4,29080^{*}$ & 1,20766 &, 003 & 1,2854 & 7,2962 \\
\hline & Gesund & Leicht-mittelschwer & $-1,22381$ & 1,15383 & ,572 & $-4,0952$ & 1,6476 \\
\hline & & Schwer & $-4,29080^{*}$ & 1,20766 &, 003 & $-7,2962$ & $-1,2854$ \\
\hline \multirow{6}{*}{$\begin{array}{l}\text { TICS- } \\
\text { Soue }\end{array}$} & Leicht-mittelschwer & Schwer & $-1,57734$ &, 74264 &, 111 & $-3,4255$ & ,2708 \\
\hline & & Gesund & ,75714 & ,73584 & ,591 & $-1,0741$ & 2,5884 \\
\hline & Schwer & Leicht-mittelschwer & 1,57734 & ,74264 &, 111 &,- 2708 & 3,4255 \\
\hline & & Gesund & $2,33448^{*}$ & ,77017 & ,013 & ,4178 & 4,2511 \\
\hline & Gesund & Leicht-mittelschwer &,- 75714 & ,73584 & ,591 & $-2,5884$ & 1,0741 \\
\hline & & Schwer & $-2,33448^{*}$ &, 77017 &, 013 & $-4,2511$ &,- 4178 \\
\hline \multirow{6}{*}{$\begin{array}{l}\text { TICS- } \\
\text { Erdr }\end{array}$} & Leicht-mittelschwer & Schwer & 3,37241 & 1,79829 & ,178 & $-1,1028$ & 7,8476 \\
\hline & & Gesund & 1,76667 & 1,78183 & 613 & $-2,6676$ & 6,2009 \\
\hline & Schwer & Leicht-mittelschwer & $-3,37241$ & 1,79829 & ,178 & $-7,8476$ & 1,1028 \\
\hline & & Gesund & $-1,60575$ & 1,86496 & 691 & $-6,2469$ & 3,0354 \\
\hline & Gesund & Leicht-mittelschwer & $-1,76667$ & 1,78183 & ,613 & $-6,2009$ & 2,6676 \\
\hline & & Schwer & 1,60575 & 1,86496 & ,691 & $-3,0354$ & 6,2469 \\
\hline \multirow{6}{*}{$\begin{array}{l}\text { TICS- } \\
\text { Unzu }\end{array}$} & Leicht-mittelschwer & Schwer & $-1,05419$ & ,88751 & ,497 & $-3,2628$ & 1,1545 \\
\hline & & Gesund & 1,06190 & ,87939 & ,485 & $-1,1265$ & 3,2503 \\
\hline & Schwer & Leicht-mittelschwer & 1,05419 & ,88751 & ,497 & $-1,1545$ & 3,2628 \\
\hline & & Gesund & 2,11609 & ,92042 & 077 &,- 1744 & 4,4066 \\
\hline & Gesund & Leicht-mittelschwer & $-1,06190$ & ,87939 & ,485 & $-3,2503$ & 1,1265 \\
\hline & & Schwer & $-2,11609$ & ,92042 & ,077 & $-4,4066$ &, 1744 \\
\hline \multirow{6}{*}{$\begin{array}{l}\text { TICS- } \\
\text { Uefo }\end{array}$} & Leicht-mittelschwer & Schwer & $-3,13005$ & 1,28084 & ,055 & $-6,3175$ & ,0574 \\
\hline & & Gesund &,- 20476 & 1,26911 & ,987 & $-3,3631$ & 2,9535 \\
\hline & Schwer & Leicht-mittelschwer & 3,13005 & 1,28084 & ,055 &,- 0574 & 6,3175 \\
\hline & & Gesund & 2,92529 & 1,32832 & ,094 &,- 3804 & 6,2309 \\
\hline & Gesund & Leicht-mittelschwer & ,20476 & 1,26911 & ,987 & $-2,9535$ & 3,3631 \\
\hline & & Schwer & $-2,92529$ & 1,32832 & ,094 & $-6,2309$ & ,3804 \\
\hline \multirow{6}{*}{$\begin{array}{l}\text { TICS- } \\
\text { Mang }\end{array}$} & Leicht-mittelschwer & Schwer & $-5,01281^{\prime}$ & 1,36126 & ,002 & $-8,4004$ & $-1,6252$ \\
\hline & & Gesund & $8,42857^{*}$ & 1,34879 &, 000 & 5,0720 & 11,7852 \\
\hline & Schwer & Leicht-mittelschwer & 5,01281 & 1,36126 & ,002 & 1,6252 & 8,4004 \\
\hline & & Gesund & $13,44138^{*}$ & 1,41172 & .000 & 9,9282 & 16,9546 \\
\hline & Gesund & Leicht-mittelschwer & $-8,42857^{\prime}$ & 1,34879 & ,000 & $-11,7852$ & $-5,0720$ \\
\hline & & Schwer & $-13,44138^{*}$ & 1,41172 & .000 & $-16,9546$ & $-9,9282$ \\
\hline \multirow{6}{*}{$\begin{array}{l}\text { TICS- } \\
\text { Sozs }\end{array}$} & Leicht-mittelschwer & Schwer & $-8,03054$ & 1,26350 &, 000 & $-11,1749$ & $-4,8862$ \\
\hline & & Gesund & $18,24762^{*}$ & 1,25193 & 000 & 15,1321 & 21,3632 \\
\hline & Schwer & Leicht-mittelschwer & 8,03054 & 1,26350 &, 000 & 4,8862 & 11,1749 \\
\hline & & Gesund & $26,27816^{*}$ & 1,31034 &, 000 & 23,0173 & 29,5391 \\
\hline & Gesund & Leicht-mittelschwer & $-18,24762$ & 1,25193 & ,000 & $-21,3632$ & $-15,1321$ \\
\hline & & Schwer & $-26,27816^{*}$ & 1,31034 &, 000 & $-29,5391$ & $-23,0173$ \\
\hline \multirow{6}{*}{$\begin{array}{l}\text { TICS- } \\
\text { Sozi }\end{array}$} & Leicht-mittelschwer & Schwer &,- 88177 & 1,54634 & ,850 & $-4,7300$ & 2,9664 \\
\hline & & Gesund & 23,59524 & 1,53218 &, 000 & 19,7823 & 27,4082 \\
\hline & Schwer & Leicht-mittelschwer & ,88177 & 1,54634 & ,850 & $-2,9664$ & 4,7300 \\
\hline & & Gesund & $24,47701^{*}$ & 1,60367 &, 000 & 20,4861 & 28,4679 \\
\hline & Gesund & Leicht-mittelschwer & $-23,59524^{\prime}$ & 1,53218 & ,000 & $-27,4082$ & $-19,7823$ \\
\hline & & Schwer & $-24,47701^{*}$ & 1,60367 &, 000 & $-28,4679$ & $-20,4861$ \\
\hline TICS- & Leicht-mittelschwer & Schwer & $-3,26798$ & 1,22023 & ,032 & $-6,3046$ &,- 2313 \\
\hline Sorg & & Gesund & $15,62857^{*}$ & 1,20905 &, 000 & 12,6197 & 18,6374 \\
\hline & Schwer & Leicht-mittelschwer & $3,26798^{*}$ & 1,22023 & 032 & ,2313 & 6,3046 \\
\hline & & Gesund & $18,89655^{*}$ & 1,26546 &, 000 & 15,7473 & 22,0458 \\
\hline & Gesund & Leicht-mittelschwer & $-15,62857^{\prime}$ & 1,20905 & ,000 & $-18,6374$ & $-12,6197$ \\
\hline & & Schwer & $-18,89655^{*}$ & 1,26546 &, 000 & $-22,0458$ & $-15,7473$ \\
\hline TICS- & Leicht-mittelschwer & Schwer & $-3,45419^{\prime}$ & ,91376 & 001 & $-5,7282$ & $-1,1802$ \\
\hline SSCS & & Gesund & 5,82857 & ,90539 &, 000 & 3,5754 & 8,0817 \\
\hline & Schwer & Leicht-mittelschwer & $3,45419^{*}$ & 91376 & ,001 & 1,1802 & 5,7282 \\
\hline & & Gesund & $9,28276^{*}$ & ,94764 &, 000 & 6,9245 & 11,6410 \\
\hline & Gesund & Leicht-mittelschwer & $-5,82857^{*}$ & ,90539 & ,000 & $-8,0817$ & $\begin{array}{l}-3,5754 \\
\end{array}$ \\
\hline & & Schwer & $-9,28276$ & ,94764 &, 000 & $-11,6410$ & $-6,9245$ \\
\hline
\end{tabular}

die Mittelwertdifferenz ist auf der Stufe 0.05 signifikant. 
Tabelle 8. Korrelationen (aus Tolic \& Soyka, 2018)

\begin{tabular}{|c|c|c|}
\hline Variable & Abstinenzdauer & Schweregrad \\
\hline Gamma-GT & $-.00^{\star \star}$ & $.04^{*}$ \\
\hline GOT & $-.00^{\star \star}$ & \\
\hline GPT & $-.00^{* *}$ & \\
\hline MCV & $-.00^{* *}$ & $.00^{\star *}$ \\
\hline Cortisol-T0 & $-.00^{* *}$ & $.02^{*}$ \\
\hline Cortisol-T1 & $-.00^{\star \star}$ & $.02^{*}$ \\
\hline Cortisol-T2 & & $.04^{*}$ \\
\hline Cortisol-T3 & & $.03^{*}$ \\
\hline Cortisol-T4 & & $.00^{\star *}$ \\
\hline Cortisol-T5 & $-.00^{\star \star}$ & $.00^{* *}$ \\
\hline EDA Beginn & $.00^{\star *}$ & \\
\hline EDA Ende & & $.00^{* *}$ \\
\hline Puls Beginn & & $.04^{*}$ \\
\hline Puls Ende & $-.01^{*}$ & $.03^{*}$ \\
\hline Angst (State-Angst) & $-.02^{*}$ & \\
\hline Angst (Trait-Angst) & $-.04^{*}$ & \\
\hline Depression (BDI II) & $-.00^{\star \star}$ & \\
\hline Trinkdauer & & $.00^{\star *}$ \\
\hline Anzahl Entzugsbehandlungen & & $.00^{* *}$ \\
\hline Anzahl Entwöhnungsbehandlungen & & $.013^{\star}$ \\
\hline Suchtdruck (MaCs) & & $.011^{*}$ \\
\hline Anzahl lebensverändernder Ereignisse & & $.00^{* *}$ \\
\hline Chronischer Stress (TICS Screening-Skala) & & $.00^{* *}$ \\
\hline $\begin{array}{l}\text { Stressverarbeitung (SVF-Positive Strategien) } \\
\text { Stressverarbeitung (SVF-Negative } \\
\text { Strategien) }\end{array}$ & $-.04^{*}$ & $\begin{array}{l}.03^{*} \\
.00^{* *}\end{array}$ \\
\hline Alltäglicher Stress (ABFB) & $.01^{*}$ & \\
\hline
\end{tabular}

${ }^{*} p<0.05$ 
Tabelle 9. Lineare Regressionsanalyse mit Cortisolwert-T5 als Kriterium (aus Tolic \& Soyka, 2018).

\section{Regression}

Eingegebene/Entfernte Variablen ${ }^{\mathrm{a}}$

\begin{tabular}{|c|c|c|c|}
\hline Modell & Eingegebene Variablen & Entfernte Variablen & Methode \\
\hline 1 & $\begin{array}{l}\text { MCV, ABFDB, SVFErs, Trinkmenge, SVFSchab, } \\
\text { SVFSitkon, SVFPha, SVFPos, TICSUefo, } \\
\text { SVFAgg, TICSUnzu, SVFSoza, TICSSozi, } \\
\text { TICSMang, SVFSozube, TICSSscs, MaCS, } \\
\text { SVFSemitl, SVFSebest, SVFAbI, STAIX2, } \\
\text { ILEgesBeIMit, SVFRekon, TICSSorg, SVFRes, } \\
\text { TICSUebe, TICSSoue, SVFEntsp, TICSErdr, } \\
\text { SVFVerm, Trinkdauer, SVFGedw, SVFFlu, } \\
\text { SVFSesch, BDI, SVFBag, SVFHer, SVFNeg, } \\
\text { SVFPosi, Schweregrade, GOT, TICSSozs, } \\
\text { STAIX1, GPT, GammaGT, Abstinenzdauer7u70b }\end{array}$ & & Aufnehmen \\
\hline
\end{tabular}

a. Abhängige Variable: CORTISOLT5

b. Alle angeforderten Variablen wurden eingegeben.

\begin{tabular}{|l|r|r|r|r|}
\hline \multicolumn{5}{|c|}{ Modellübersicht $^{\mathbf{b}}$} \\
\hline Modell & $\mathrm{R}$ & R-Quadrat & $\begin{array}{c}\text { Angepasstes R- } \\
\text { Quadrat }\end{array}$ & $\begin{array}{l}\text { Standardfehler } \\
\text { der Schätzung }\end{array}$ \\
\hline 1 &, $966^{\mathrm{a}}$ &, 934 &, 754 & 1,31696 \\
\hline
\end{tabular}

a. Prädiktoren: (Konstante), MCV, ABFDB, SVFErs, Trinkmenge,

SVFSchab, SVFSitkon, SVFPha, SVFPos, TICSUefo, SVFAgg,

TICSUnzu, SVFSoza, TICSSozi, TICSMang, SVFSozube, TICSSscs,

MaCS, SVFSemitl, SVFSebest, SVFAbl, STAIX2, ILEgesBelMit,

SVFRekon, TICSSorg, SVFRes, TICSUebe, TICSSoue, SVFEntsp,

TICSErdr, SVFVerm, Trinkdauer, SVFGedw, SVFFlu, SVFSesch, BDI, SVFBag, SVFHer, SVFNeg, SVFPosi, Schweregrade, GOT, TICSSozs,

STAIX1, GPT, GammaGT, Abstinenzdauer7u70

b. Abhängige Variable: CORTISOLT5

\begin{tabular}{|ll|r|r|r|r|r|}
\hline \multicolumn{2}{|c|}{ ANOVA $^{\text {a }}$} & & \\
Modell & & Quadratsumme & df & $\begin{array}{l}\text { Mittel der } \\
\text { Quadrate }\end{array}$ & F & Sig. \\
\hline 1 & Regression & 414,479 & 46 & 9,010 & 5,195 &, $000^{\mathrm{b}}$ \\
& Residuum & 29,484 & 17 & 1,734 & & \\
& Gesamtsumme & 443,964 & 63 & & & \\
\hline
\end{tabular}

a. Abhängige Variable: CORTISOLT5

b. Prädiktoren: (Konstante), MCV, ABFDB, SVFErs, Trinkmenge, SVFSchab, SVFSitkon, SVFPha, SVFPos, TICSUefo, SVFAgg, TICSUnzu, SVFSoza, TICSSozi, TICSMang, SVFSozube, TICSSscs, MaCS, SVFSemitl, SVFSebest, SVFAbI, STAIX2, ILEgesBelMit, SVFRekon, TICSSorg, SVFRes, TICSUebe, TICSSoue, SVFEntsp, TICSErdr, SVFVerm, Trinkdauer, SVFGedw, SVFFlu, SVFSesch, BDI, SVFBag, SVFHer, SVFNeg, SVFPosi, Schweregrade, GOT, TICSSozs, STAIX1, GPT,

GammaGT, Abstinenzdauer7u70 